\title{
Summary and Recommendations: Total Fuel Cycle Assessment Workshop
}

$B C S$, Incorporated

Columbia, Maryland

NREL Technical Monitor: Jim Ohi

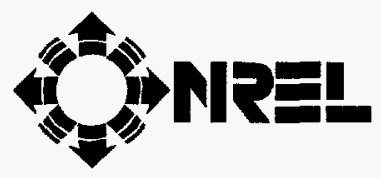

National Renewable Energy Laboratory 1617 Cole Boulevard

Golden, Colorado 80401-3393

A national laboratory of the U.S. Department of Energy Managed by Midwest Research Institute for the U.S. Department of Energy under Contract No. DE-AC36-83CH10093

Prepared under Subcontract No.AAW-4-14256-01 August 1995 
This publication was reproduced from the best available camera-ready copy submitted by the subcontractor and received no editorial review at NREL.

\section{NOTICE}

This report was prepared as an account of work sponsored by an agency of the United States government. Neither the United States government nor any agency thereof, nor any of their employees, makes any warranty, express or implied, or assumes any legal liability or responsibility for the accuracy, completeness, or usefulness of any information, apparatus, product, or process disclosed, or represents that its use would not infringe privately owned rights. Reference herein to any specific commercial product, process, or service by trade name, trademark, manufacturer, or othenwise does not necessarily constitute or imply its endorsement, recommendation, or favoring by the United States government or any agency thereof. The views and opinions of authors expressed herein do not necessarily state or reflect those of the United States government or any agency thereof.

Available to DOE and DOE contractors from:

Office of Scientific and Technical Information (OSTI)

P.O. Box 62

Oak Ridge, TN 37831

Prices available by calling (615) 576-8401

Available to the public from:

National Technical Information Service (NTIS)

U.S. Department of Commerce

5285 Port Royal Road

Springfield, VA 22161

(703) $487-4650$ 


\section{DISCLAIMER}

Portions of this document may be illegible in electronic image products. Images are produced from the best available original document. 


\section{Foreword}

The Total Fuel Cycle Assessment Workshop was organized to address some of the key methodological and application issues surrounding the practice of fuel cycle assessment. In so doing, the workshop brought together a diverse group of energy planners, modelers, and decision makers to provide expert insights into these issues. Workshop participants clearly stated that there is a need for an analytical method to perform full and consistent comparisons of energy conversion technologies throughout the total fuel cycle of an energy resource.

The National Renewable Energy Laboratory thanks all of those individuals who participated in the workshop and made it a success and is grateful to the University of Texas for its assistance and hospitality in hosting the workshop. Appreciation is also extended to the U.S. Department of Energy, Office of Utility Technologies, Integrated Resource Planning Program and to the Gas Research Institute for sponsoring the workshop. 
SUMMARY AND RECOMMENDATIONS $\ldots \ldots \ldots \ldots \ldots \ldots \ldots$

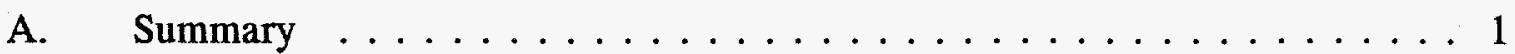

B. Recommendations $\ldots \ldots \ldots \ldots \ldots \ldots \ldots \ldots$

PROCEEDINGS: TOTAL FUEL CYCLE ASSESSMENT WORKSHOP . . . . . . . . A-1

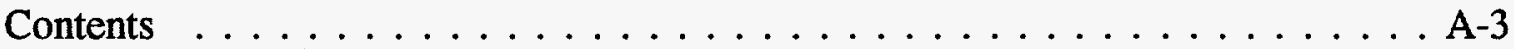

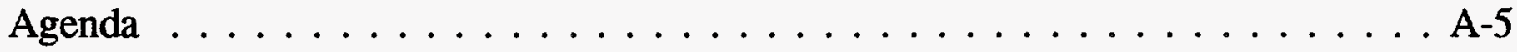

List of Attendees $\ldots \ldots \ldots \ldots \ldots \ldots \ldots \ldots \ldots \ldots \ldots \ldots$ A $9 \ldots \ldots$

Presentations and Papers $\ldots \ldots \ldots \ldots \ldots \ldots \ldots \ldots$ A-17

Status of Current Fuel Cycle Research $\ldots \ldots \ldots \ldots \ldots \ldots$. . . . . . . .

Establishing Appropriate Boundaries and Parameters for

Total Fuel Cycle Assessment . . . . . . . . . . . . . . . . . A-87

Utility Applications of Total Fuel Cycle Analysis in Planning . . . . . . A-133

Summary Sessions: Key Points . . . . . . . . . . . . . . . . . . . . A-229 


\section{SUMMARY AND RECOMMENDATIONS TOTAL FUEL CYCLE ASSESSMENT WORKSHOP}

\section{A. SUMMARY}

The Total Fuel Cycle Assessment Workshop, held in Austin, Texas October 6 - 7, 1994 was successful in bringing together nearly 60 individuals from a broad spectrum of organizations and viewpoints to discuss the merits of total fuel cycle analysis (TFCA) and its potential applications.

The workshop got off to a good start with a welcoming address from Commissioner Karl Rabago from the Public Utility Commission of Texas. He stressed the need for tools to improve the resource of information available to state regulators and policy makers. Appropriately designed TFCA tools are one method of fulfilling that need. Also, Commissioner Rabago stressed the need for the energy industry to "clean up after yourselves" and use tools such as TFCA to reach that end. Commissioner Rabago's remarks set the tone for the workshop. Speakers and participants alike began to discuss how the electric and gas utility industries could begin to do a better job of addressing a range of impacts. This resulted in many thoughtful discussions regarding energy resource selection processes and improving the quality of information available to decision makers.

During the opening session, presentations were given on four TFCA tools that have been developed or are in the developmental stages. Participants were struck with the variety of TFCA tools being developed, which included tools focusing on cradle to grave resource energy efficiency for electric and gas utilities, tools for framing fuel cycle analysis, expert systems, and research on environmental damages within a specific region. This session set the stage for the remainder of the two day workshop. It acted to broaden the perspectives of many participants on the range of potential applications for TFCA.

Throughout the two-day workshop, there was lively discussion on many of the policy and technical issues surrounding TFCA as a tool. Utility representatives spoke on their current use of analytical tools which focus on regulated source emissions from the power plant and not the range of other fuel cycle costs and benefits. However, many additional costs and benefits were identified by participants as important to evaluate.

Significant discussion focused on the potential applications for TFCA and whether or not there was a need for TFCA in energy planning. Opinions regarding the need for TFCA varied. Some participants felt that TFCA could be useful for identifying the energy, economic, and environmental costs and benefits of various technologies. Other participants felt that there are models and techniques already available to meet regulatory requirements for disclosure by utilities. 
Many of the representatives from the gas industry indicated that TFCA should be used to analyze source efficiency of alternative energy options or end-use technologies. State representatives indicated that TFCA could be useful in improving the level and quality of information available to them in support of their decision making processes. Also, they indicated that TFCA could be a useful tool in estimating economic development impacts from alternative energy planning scenarios and that this would be very important to them. In general, electric industry representatives indicated that TFCA should not be included as part of IRP but that some of the information that TFCA generates could be useful to augment existing decision making processes.

One of the highlights of the workshop was the insightful discussion on the value that such tools can provide to state regulators and policy makers. This included addresses and presentations by Karl Rabago, Robert Early, Chairman of the Committee on Energy Resources, Texas State Legislature, Dr. Ajay Sanghi, New York State Energy Office, and Thomas Tanton, California Energy Commission. The value of TFCA as a tool to assist decision makers was corroborated by these state representatives and a number of other participants.

There was consensus on some points including: the added level of information provided by tools such as TFCA could assist decision makers; if pursued, TFCA tools should be fair and objective to all resources; data quality needs to be improved; and that efforts should be made to reduce the complexity of implementing TFCA and to make TFCA tools cost effective to implement. Various potential applications for TFCA also were identified. Many of the tools already in use that look at components of the fuel cycle should be considered for integration within a TFCA effort. Also, there was significant discussion in terms of the specific boundary and modeling questions that were asked of participants. Here several suggested approaches and activities were recommended for the U.S. Department of Energy to pursue.

\section{B. DRAFT RECOMMENDATIONS}

The final session of the workshop, Putting it All Together, provided participants with a summary of the previous sessions followed by an open discussion with participants to identify common ground and common needs as well as identify potential next steps.

Key recommendations of activities for DOE to consider follow:

1. Establish a Fuel Cycle Assessment Focus Group - This activity would be to create a focus group on how to proceed with TFCA. Participants should be a small but targeted group of representatives from state and federal government; electric and gas utilities; oil, coal, gas, nuclear, and renewables industries; and other TFCA stakeholders. Some of the issues that the focus group could address include: 
- Definition of terms,

- Data needs, and

- TFCA approaches and applications (building off the results of the workshop),

2. Conduct an Assessment of Alternative TFCA Approaches - Throughout the workshop, alternative approaches and applications were identified for TFCA. It was recommended that an assessment of the various TFCA approaches be made based on their individual qualities/criteria. Examples of assessment qualities/criteria are shown below.

\begin{tabular}{|l|l|l|l|l|l|}
\hline Qualities & Applications & $\begin{array}{c}\text { Criteria Impacts } \\
\text { Evaluated } \\
\text { (Economic, } \\
\text { Environmental, } \\
\text { etc.) }\end{array}$ & $\begin{array}{c}\text { Fuel } \\
\text { Cycle } \\
\text { Stages }\end{array}$ & $\begin{array}{c}\text { Time to } \\
\text { Implement }\end{array}$ & $\begin{array}{c}\text { Cost to } \\
\text { Implement }\end{array}$ \\
\hline Approach No. 1 & & & & & \\
\hline Approach No. 2 & & & & & \\
\hline Approach No. 3 & & & & & \\
\hline
\end{tabular}

Alternative approaches could be identified. The NREL compendium could be used as a starting point and additional international, national, state and local approaches could be added. A thorough evaluation would be performed of each approach. This would provide potential users the ability to identify the approach(es) best suited for their own needs as well as assist TFCA modelers identify key qualities that should be resident in any approach.

Clear approaches are needed for performing TFCA. They will depend on the specific objectives and applications of the user, impacts being assessed, and boundaries being established. A typology of approaches or TFCA framework could be developed that is adaptable to various applications and locales. This activity could include a survey on the needs of energy planners and policy makers to determine their needs in terms of data, tools, information etc. that will assist them in their decision making.

3. Further Analysis of the Models Presented at the workshop - Some of the leading TFCA activities in the country were presented at the workshop. Due to the extent of information covered during the two days, there was not enough time to evaluate each of these models in depth. This effort would examine each of these models in further detail to identify the range of potential applications, stage of development, current applications, and other characteristics. 
4. Identify/Develop Data Elements to Support TFCA - There is a lack of data available for performing TFCA. Key data elements should be identified and measures taken to increase the availability of such data. This task would work with key TFCA practitioners to identify current gaps in data resources and to identify key gaps which need to be filled. This activity should also identify methods for collecting these data. 


\author{
Austin, Texas \\ October 5-6, 1994 \\ Prepared for: \\ National Renewable Energy Laboratory \\ 1617 Cole Boulevard \\ Golden, Colorado 80401 \\ and \\ U.S. Department of Energy \\ Office of Energy Efficiency and Renewable Energy \\ Office of Utility Technologies \\ Integrated Resource Planning Program \\ 1000 Independence Ave., SW \\ Washington, D.C. 20585
}

\author{
Prepared by: \\ BCS, Incorporated \\ 5550 Sterrett Place, Suite 216 \\ Columbia, Maryland 21044 \\ ph. (410) $997-7778$ \\ fax (410) $997-7669$
}

Energy, Environment, and Economic Analysis 



\section{CONTENTS}

AGENDA $\quad$ A-5

LIST OF ATTENDEES $\quad$ A-9

$\begin{array}{ll}\text { PRESENTATIONS AND PAPERS } & \text { A-17 }\end{array}$

Status of Current Fuel Cycle Research A-19

- Fuel Cycle Assessment Guide for State and Utility Planners A-21

- ASPEN as a Total Fuel Cycle Modeling Tool A-41

- Think Globally, Calculate Locally: The American Gas Cooling

Center Resource Energy Efficiency and Emissions Database and Modeling Program

The New York Environmental Externalities Cost Study A-61

Session No. 1: Establishing Appropriate Boundaries and Parameters

for Total Fuel Cycle Assessment

Electric Vehicle Total Energy Cycle Assessment

Session No. 2: Identifying Appropriate Models, Methods and Approaches for Implementing TFCA

- $\quad$ Source Energy Analysis: Total Fuel Cycle Assessment

Session No. 3: Utility Applications of TFCA in Planning

A-133

- $\quad$ Total Fuel Cycle Analysis: Learning from the California Experience

- Applications in New York State

A-152

- $\quad$ Total Fuel Cycle Assessment: Gas Utility Applications

Session No. 4: State Regulatory and Planning Perspectives on TFCA

- Total Fuel Cycle Assessment Workshop: Comments by State Representative Robert Earley

Multi-Stage Emission Accounting: New York Experience 


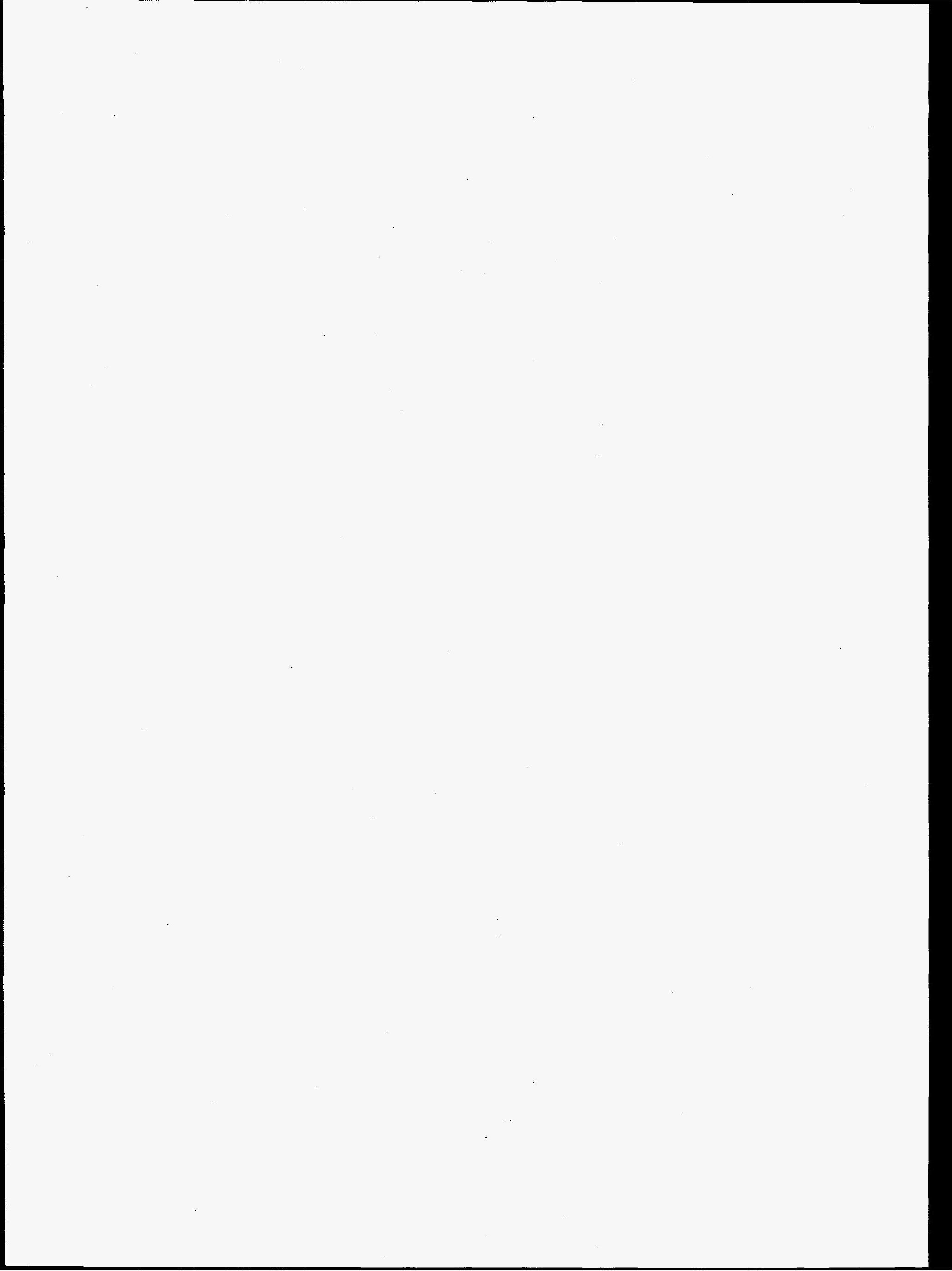


AGENDA

$$
\text { A-5 }
$$



$7: 30-8: 30$

$8: 30-9: 00$

$9: 00-9: 10$

$9: 10-9: 40$

$9: 40-10: 10$

$10: 10-10: 30$

$10: 30-11: 00$

$11: 00-11: 30$

$11: 30-12: 00$

$12: 00-1: 00$

$1: 00-3: 15$

Registration (Continental Breakfast)

Welcome - Karl Rabago, Commissioner, Texas Public Utilities Commission

Workshop Overview - Jim Ohi, National Renewable Energy Laboratory

STATUS OF CURRENT FUEL CYCLE RESEARCH

Moderator: Jim Ohi, National Renewable Energy Laboratory

Fuel Cycle Assessment Guide for State and Utility Planners: Establishing

Parameters and Identifying Applications in the State of Maryland - Kenneth

M. Green, BCS, Incorporated

ASPEN Model - Steven Adelman, National Renewable Energy Laboratory

Break

Resource Energy Efficiency and Emissions Model - Dr. Michael Witte, ElectroCom GARD

New York Environmental Externalities Cost Study - Robert Rowe, RCG/Hagler Bailly

Open Discussion and follow-on Q\&A for Morning Panel

Complimentary Lunch

WORKSHOP SESSIONS: DEVELOPING TFCA APPROACHES

No. 1: Establishing Appropriate Boundaries and Parameters for Total Fuel Cycle Assessment. Opening Presentation: Electric Vehicle Total Energy

Cycle Analysis, Jim Ohi, National Renewable Energy Laboratory

Facilitators: Kenneth W. Boras, BCS, Incorporated; Michael Samsa, Gas Research Institute

3:15-3:30 Break

$3: 30-5: 45$

No. 2: Identifying Appropriate Models, Methods and Approaches for Implementing Total Fuel Cycle Assessment. Opening Presentation: Electric and Gas Cooling, James Block, Xenersys

Facilitators: Jay Zarnikau, Planergy; Mark Krebs, Managing Director, Southern Union Energy International

7:00 - 9:00 


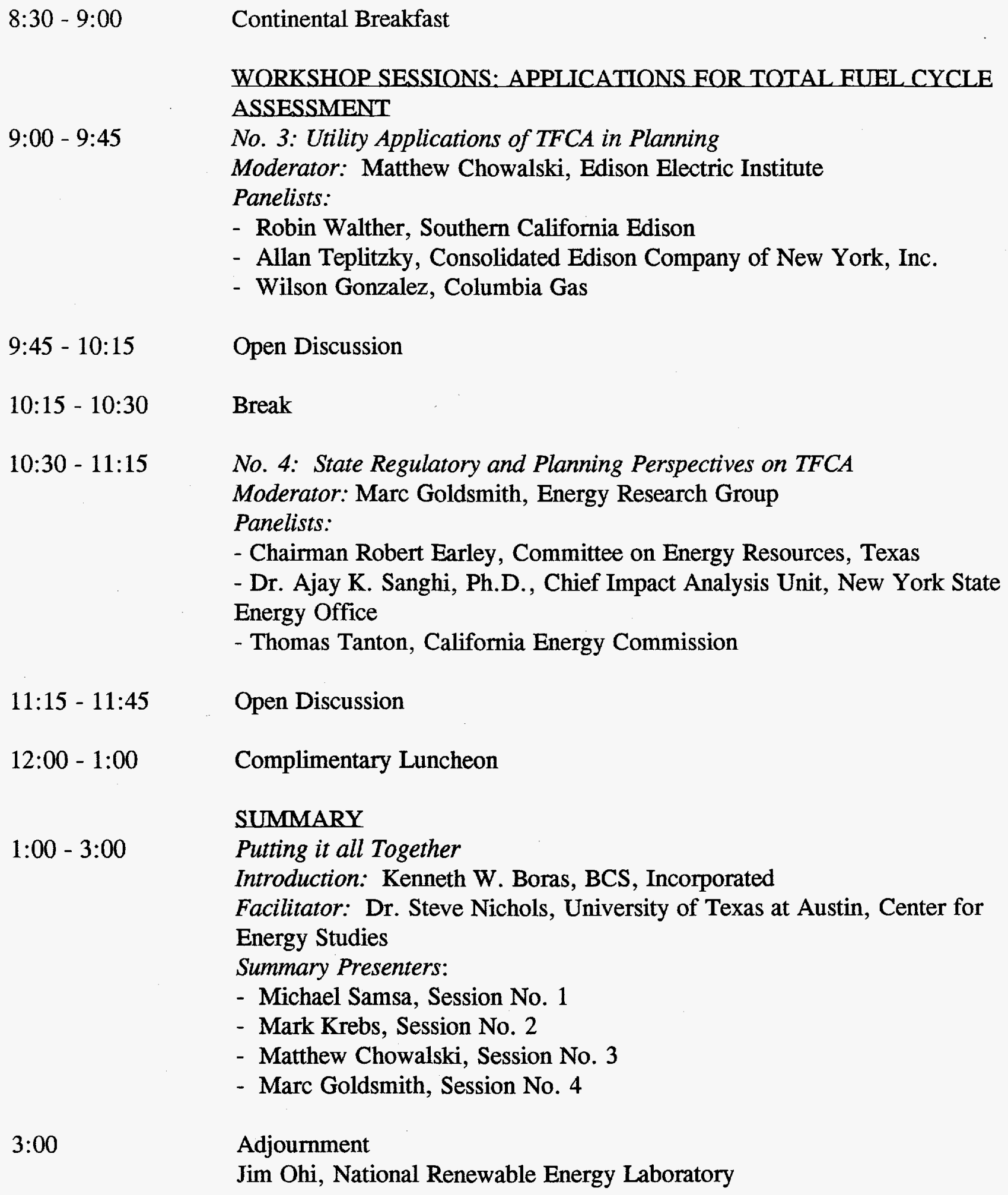

- Chairman Robert Earley, Committee on Energy Resources, Texas

- Dr. Ajay K. Sanghi, Ph.D., Chief Impact Analysis Unit, New York State Energy Office

- Thomas Tanton, California Energy Commission

11:15-11:45 Open Discussion

12:00 - 1:00 Complimentary Luncheon

1:00 - 3:00 Putting it all Together

Introduction: Kenneth W. Boras, BCS, Incorporated

Facilitator: Dr. Steve Nichols, University of Texas at Austin, Center for Energy Studies

Summary Presenters:

- Michael Samsa, Session No. 1

- Mark Krebs, Session No. 2

- Matthew Chowalski, Session No. 3

- Marc Goldsmith, Session No. 4 
LIST OF ATTENDEES 



\section{TOTAL FUEL CYCLE ASSESSMENT WORKSHOP ATTENDEES}

Steve Adelman

National Renewable Energy Laboratory

1617 Cole Blvd.

Golden, CO 80401

ph. (303) 275-4617

Gordon Alexander

City of Austin, ECSD

206 E. 9th St., Ste. 17.102

Austin, TX 78701

ph. (512) 499-3569

fx. (512) 499-2859

Jim Block

Xenersys Research Group

ph. (713) 482-2368

fx. (713) $482-4632$

Kenneth W. Boras

BCS, Incorporated

5550 Sterrett Pl., Ste. 216

Columbia, MD 21044

ph. (410) 997-7778

fx. (410) $997-7669$

Donald Brundage

Southern Company Services

64 Perimeter Center East

Atlanta, GA 30346

ph. (404) 668-4667

fx. (404) 668-4673

Peter Caffrey

U.S. EPA

2505 Plymouth Rd.

Ann Arbor, MI 48118

ph. (313) 741-7829

fx. (313) 668-4368
Charlotte Cheng

Resource Planning

Tennessee Valley Authority

1101 Market St., MB-3K

Chattanooga, TN 37402

ph. (615) 751-8767

fx. (615) 751-6128

Matt Chwalowski

Edison Electric Institute

701 Pennsylvania Ave., NW

Washington, D.C. 20004

ph. (202) 5085554

fx. (202) 508-5335

Jeff Cook

Texas General Land Office

1700 N. Congress Ave., Ste. 832

Austin, TX 78701

ph. (512) 463-9989

fx. (512) $475-1558$

Cynthia Cordova

American Gas Association

1515 Wilson Blvd.

Arlington, VA 22209

ph. (703) 841-8495

fx. (703) 841-8689

Kenneth G. Darrow

Energy International, Inc.

127 Bellevue Way, S.E., Ste. 200

Bellevue, WA 98004-6229

ph. (208) 453-9595

fx. (208) 455-0981 
David DeAngelo

Pennsylvania Power and Light Co

2 North Ninth St.

Allentown, PA 18101

ph. (215) 774-4154

fx. (215) 774-5141

Armando deLeon

City of Austin, ECSD

206 E. 9th St., Ste. 17.102

Austin, TX 78701

ph. (512) 499-3598

fx. (512) 499-2859

Jason Duncan

City of Austin, ECSD

206 E. 9th St., Ste. 17.102

Austin, TX 78701

ph. (512) 499-2513

fx. (512) 499-2859

Chairman Robert Earley

Committee on Energy Resources

Texas State Legislature

P.O. Box 2910

Austin, TX 78768

ph. (512) 463-0512

fx. (512) 463-6270

Marc Goldsmith

Energy Research Group, Inc.

400 Fifth Ave.

Waltham, MA 02154

ph. (617) 890-1730

fx. (617) 890-5094

Wilson Gonzalez

Columbia Gas Distribution Co.

200 Civic Center Dr.

P.O. Box 117

Columbus, OH 43216-0117

ph. (614) 460-6220

fx. (614) 460-6442
Kenneth M. Green

BCS, Incorporated 5550 Sterrett PI., Ste. 216

Columbia, MD 21044

ph. (410) $997-7778$

fx. (410) 997-7669

William F. Hederman

R.J. Rudden Associates

1110 North Glebe Rd., Ste. 725

Arlington, VA 22201

ph. (703) 812-8500

fx. (703) $812-8502$

Henry Herbert

Entergy Services, Inc.

P.O. Box 3689

Beaumont, TX 77704

ph. (409) 827-5132

fx. (409) 827-5292

Steve Jaeger

Railroad Commission of Texas

Alternative Fuels Division

P.O. Box 12967

Austin, TX 78711

ph. (512) 463-9202

fx. (512) 463-7292

Charles Jenkins, III

TU Electric Co.

400 North Olive St., LB 81

Dallas, TX 75201

ph. (214) 812-8265

fx. (214) $812-8685$

Mark Krebs

Southern Union Energy International

504 Lavaca, Ste. 900

Austin, TX 78701

ph. (512) 370-8208

fx. (512) $370-8245$ 
Robert Lawhn

Houston Lighting \& Power Company

12301 Kurland

Houston, TX 77034

ph. (713) $945-8207$

fx. (713) $945-7598$

Ned Leonard

Western Fuels Assn., Inc.

1625 M St., NW

Washington, D.C. 20036-3264

ph. (202) 463-6580

fx. (202) 223-8790

\section{Maggie Mann}

National Renewable Energy Laboratory

1617 Cole Blvd.

Golden, CO 80401

ph. (303) 275-2921

fx. (303) 275-2905

Jennifer Martin

DynCorp-Meridian

4300 King St., Ste. 400

Alexandria, VA 22314

ph. (703) 998-3712

fx. (703) 998-0887

Adan Martinez

Environmental Defense Fund

1800 Guadalupe

Austin, TX 78701

ph. (512) 478-5161

fx. (512) $478-8140$

Thomas W. McBroom

Atlanta Gas Light Co.

P.O. Box 4569

Atlanta, GA 30302

ph. (404) 584-3842

fx. (404) 584-3717
John McElroy, Jr.

Office of Public Utility Counsel

7800 Shoal Creek Blvd., Ste. 290E

Austin, TX 78757

ph. (512) 475-3700

fx. (512) 475-3707

Gary Miller

Edison Electric Institute

701 Pennsylvania Ave., NW

Washington, D.C. 20004

ph. (202) 508-5173

fx. (202) 508-5225

Dr. Steve Nichols

Univ. of Texas at Austin

10100 Burnet Rd., EME-133

Austin, TX 78758-4497

ph. (512) $471-4496$

Victor Niemeyer

EPRI

3412 Hillview Ave.

Palo Alto, CA 94304

ph. (415) $855-2744$

fx. (415) $855-2950$

Jim Ohi

National Renewable Energy Laboratory

1617 Cole Blvd.

Golden, CO 80401

ph. (303) 275-4681

fx. (303) $275-4695$

Karl Rabago, Commissioner

Public Utility Commission of TX

7800 Shoal Creek Blvd.

Austin, TX 78757

ph. (512) 458-0293

fx. (512) 458-0391

Tom Rose

TU Electric

1601 Bryan, Ste. 3200

Dallas, TX 75201-3411

ph. (214) 812-3247

fx. (214) 812-3201 
Terry G. Ross

Center for Energy and Economic

Development

7853 E. Arapahoe Ct., Ste. 2600

Englewood, CO 80112

ph. (303) 694-4244

fx. (303) 694-4299

Robert Rowe

RCG/Hagler Bailly

P.O. Drawer O

Boulder, CO 80806-1906

ph. (303) 449-5515

fx. (303) 443-5684

Michael Samsa

Gas Research Institute

8600 West Bryn Mawr Ave.

Chicago, IL 60631

ph. (312) 399-8156

fx. (312) $399-8170$

Dr. Ajay K. Sanghai, Ph.D.

Chief Impact Analysis Unit

2 Empire State Plaza, Ste. 1801

Albany, NY 12223-1252

ph. (518) 474-7690

fX. (518) 473-8687

John A. Shugars

DOE/FEMP, Program Office

LBL

1250 Maryland Ave., SW

Suite 150

Washington, D.C. 20024

ph. (202) 484-0880

fX. (202) 484-0888

Ronald L. Smith

Allegheny Power Systems

800 Cabin Hill Dr.

Greensburg, PA 15601

ph. (412) 838-3067

fx. (412) 838-6888
Richard Sweetser

American Gas Cooling Center

1515 Wilson Blvd.

Arlington, VA 22209

ph. (703) 841-8411

fx. (703) 841-8606

Thomas Tanton

California Energy Commission 1516 Ninth St.

Sacramento, CA 95814-5512

ph. (916) 654-3787

fx. (916) 654-4420

Alan R. Tarrant

Lone Star Gas Co.

301 S. Harwood

Dallas, Texas 75201

ph. (214) 573-3866

fx. (214) $573-5172$

Allan Teplitzky

Consolidated Edison

4 Irving Pl.

New York, NY 10003

ph. (212) 460-4859

fx. (212) $982-8194$

Nicholas Tibberts

American Electric Power Service Corp.

1 Riverside Plaza

Columbus, OH 43215

ph. (614) 223-2303

fx. (614) 223-2352

Andrew Varley

American Electric Power Service Corp.

1 Riverside Plaza

Columbus, OH 43215

ph. (614) 223-2800

fx. (614) 223-2807 
Robin Walther

Southern California Edison 2244 Walnut Grove

Rosewood, CA 91105

ph. (818) 302-9653

fx. (818) 302-4841

Michael J. Witte

ElectroCom GARD

7449 North Natchez Ave.

Niles, IL 60714-3892

ph. (708) 647-3254

fx. (708) 647-8678

Jay Zarnikau

Planergy, Inc.

1008 West Ave.

Austin, TX 78701

ph. (512) 477-8012

fx. (512) 477-2149 



\section{PRESENTATIONS AND PAPERS}





\title{
A FUEL CYCLE ASSESSMENT GUIDE FOR UTILITY AND STATE ENERGY PLANNERS
}

October 6, 1994

\author{
Prepared for: \\ Total Fuel Cycle Assessment (TFCA) Workshop \\ Austin, Texas
}

\author{
Presented by: \\ Kenneth M. Green \\ BCS, Incorporated \\ 5550 Sterrett Place, Suite 216 \\ Columbia, Maryland 21044 \\ ph. (410) $997-7778$ \\ $\operatorname{fax}(410)$ 997-7669
}

Energy, Environment, and Economic Analysis 


\section{OBJECTIVES OF THE GUIDE}

- THE FUEL CYCLE ASSESSMENT GUIDE IS A SYSTEMATIC TOOL FOR FRAMING LOCALLY APPROPRIATE TFCA

- THE GUIDE CAN BE USED FOR IRP, ENERGY STRATEGY DEVELOPMENT AND A RANGE OF OTHER APPLICATIONS 


\section{PROJECT BACKGROUND}

- THE GUIDE WAS DEVELOPED BY BCS FOR THE U.S. DEPARTMENT OF ENERGY, IRP PROGRAM UNDER THE DIRECTION OF THE NATIONAL RENEWABLE ENERGY LABORATORY

- THE GUIDE WAS TESTED AS A TFCA FRAMING TOOL IN THE STATE OF MARYLAND DURING 1993

- KEY OBJECTIVES:

- To Improve the Framing Function of the Guide, and

- To Identify Data Resources Available by Stage and by Category of Cost/Benefit 


\section{STRAIGHTFORWARD APPROACH}

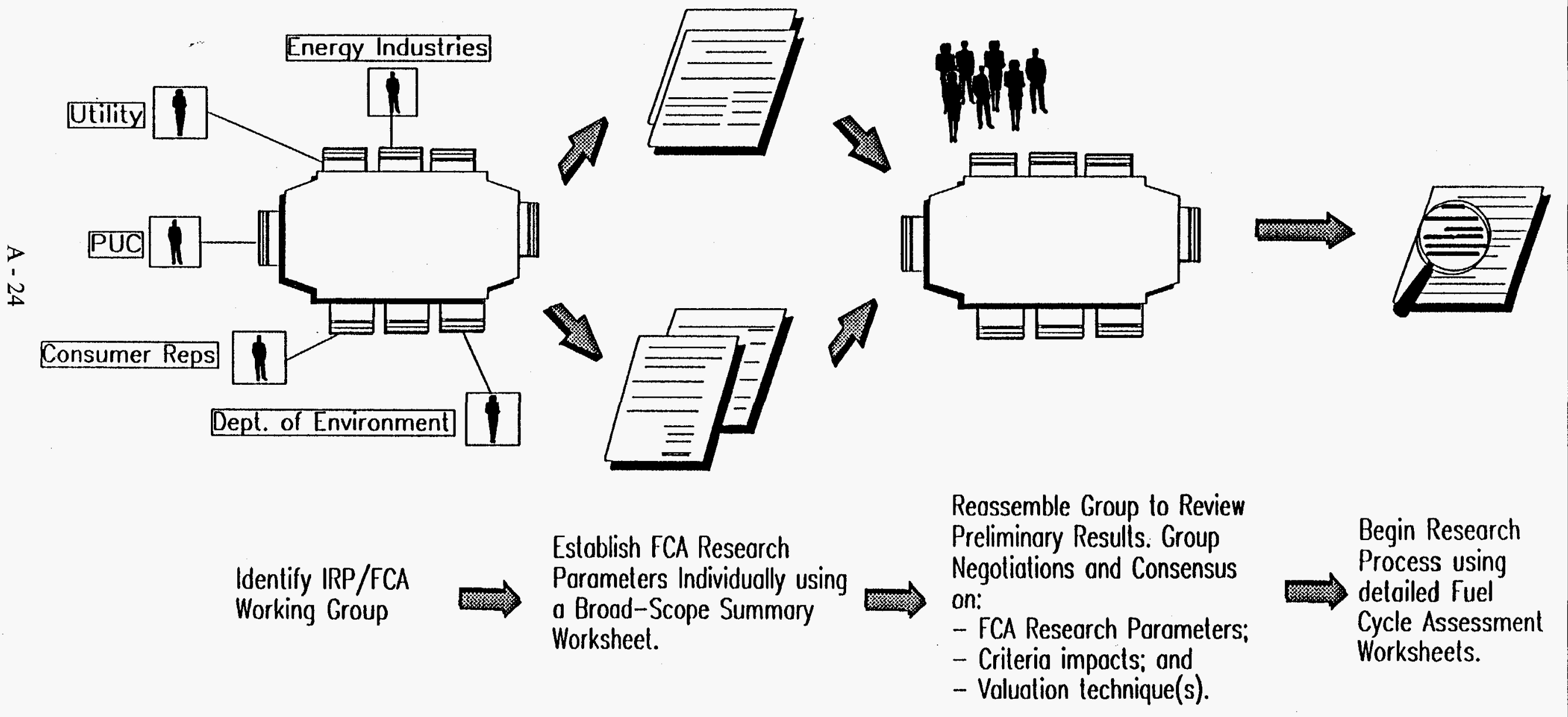




\section{MARYLAND WORKING GROUP}

- THE GUIDE WAS TESTED USING A WORKING GROUP APPROACH.

PARTICIPANTS INCLUDED:

$\Rightarrow$ Maryland State Energy Office

$\Rightarrow$ Maryland Public Service Commission

$\rightarrow$ Maryland Department of Natural Resources

$\Rightarrow$ Potomac Electric Power Company

$\Rightarrow$ Baltimore Gas \& Electric

$\rightarrow$ Maryland Coal Association

$\Rightarrow$ Buffalo Coal

$\Rightarrow$ Columbia Gas

$\Rightarrow$ Osram Sylvania

$\Rightarrow$ Solarex

$\Rightarrow \quad$ National Renewable Energy Laboratory 


\section{SELECTED CASE EXAMPLES}

- FOUR CASE EXAMPLES SELECTED BY THE WORKING GROUP:

- $\quad$ Combined Cycle Gas Turbine System

- Pulverized Coal Cogeneration Applications

- Energy Efficient Lighting Retrofit

- $\quad$ Photovoltaics for use in Augmenting Power Supply at an Aquaculture Facility 
Supply Technology Application

\begin{tabular}{|l||}
\hline Overview: \\
\hline Location/Acreage - \\
\hline System type/Supplier - \\
\hline Prime Mover/Back-up - \\
\hline \\
System size - \\
\hline Fuel supply/source - \\
\hline Anticipated date on-line - \\
\hline - Other \\
\hline Operational Data: \\
\hline Anticipated annual generation \\
\hline Ieat rate (Btu/kWh) \\
\hline Net efficiency \\
\hline Emission rates \\
\hline Duty Cycle \\
\hline Emission control technologies \\
\hline Water consumption \\
\hline Steam production \\
\hline - Other \\
\hline Cost Data: \\
\hline Capital costs (estimated) \\
\hline O\&M costs (estimated) \\
\hline - Fixed \\
\hline - Variable \\
\hline - Other \\
\hline
\end{tabular}

Demand-Side Technology Application

\begin{tabular}{|l|l|}
\hline Overview: & Description \\
\hline Project/Site - \\
\hline Technology/Supplier - \\
\hline Size/Quantity - \\
\hline Anticipated installation date - \\
\hline Anticipated life-cycle \\
\hline - Other \\
\hline Operational Data: \\
\hline Anticipated annual energy \\
\hline consumption \\
\hline kW/therms avoided \\
\hline Net efficiency \\
\hline Emissions avoided \\
\hline Duty cycle \\
\hline - Other \\
\hline Cost Data: \\
\hline Capital costs (estimated) \\
\hline O\&M costs (estimated) \\
\hline - Fixed \\
\hline
\end{tabular}




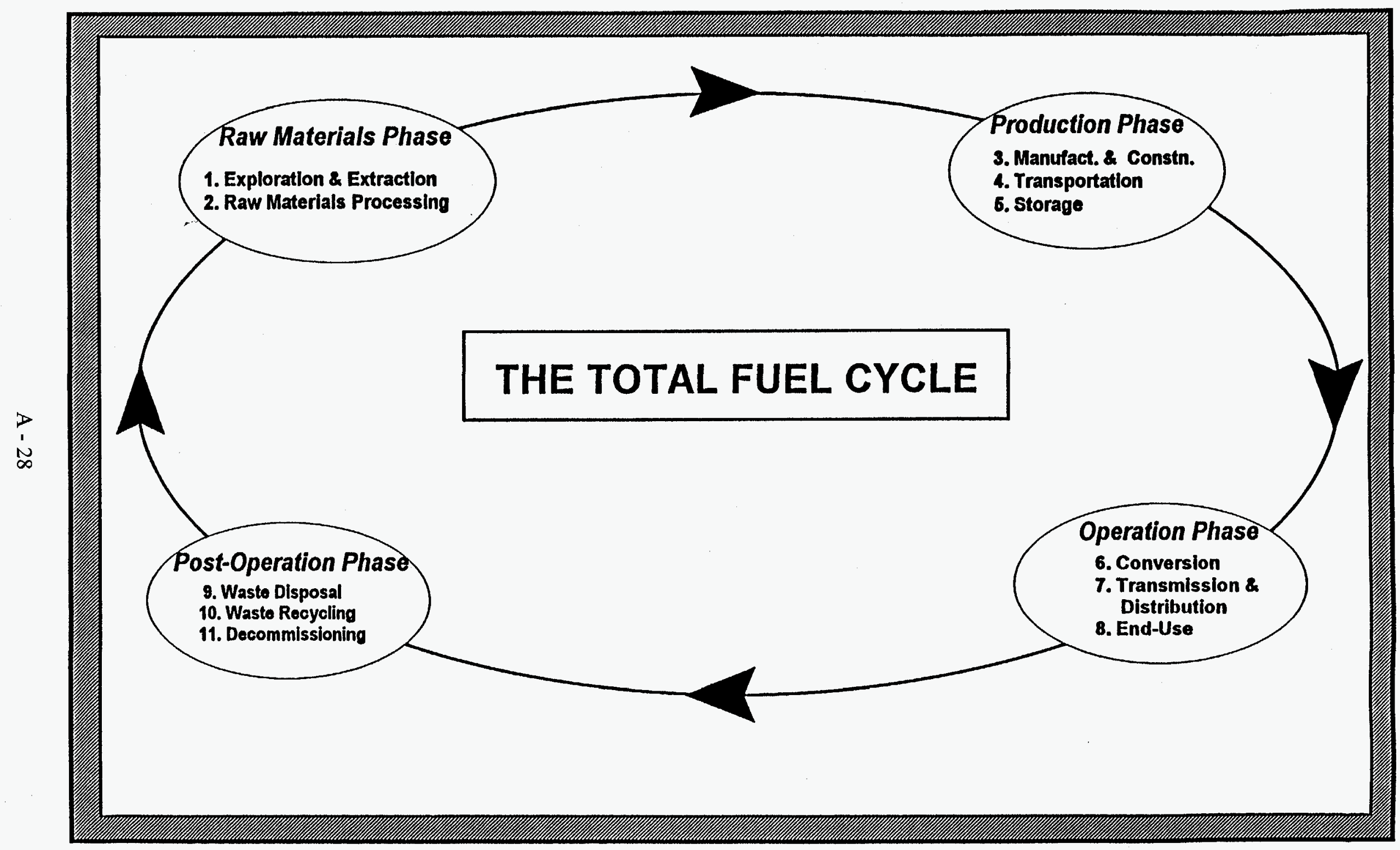




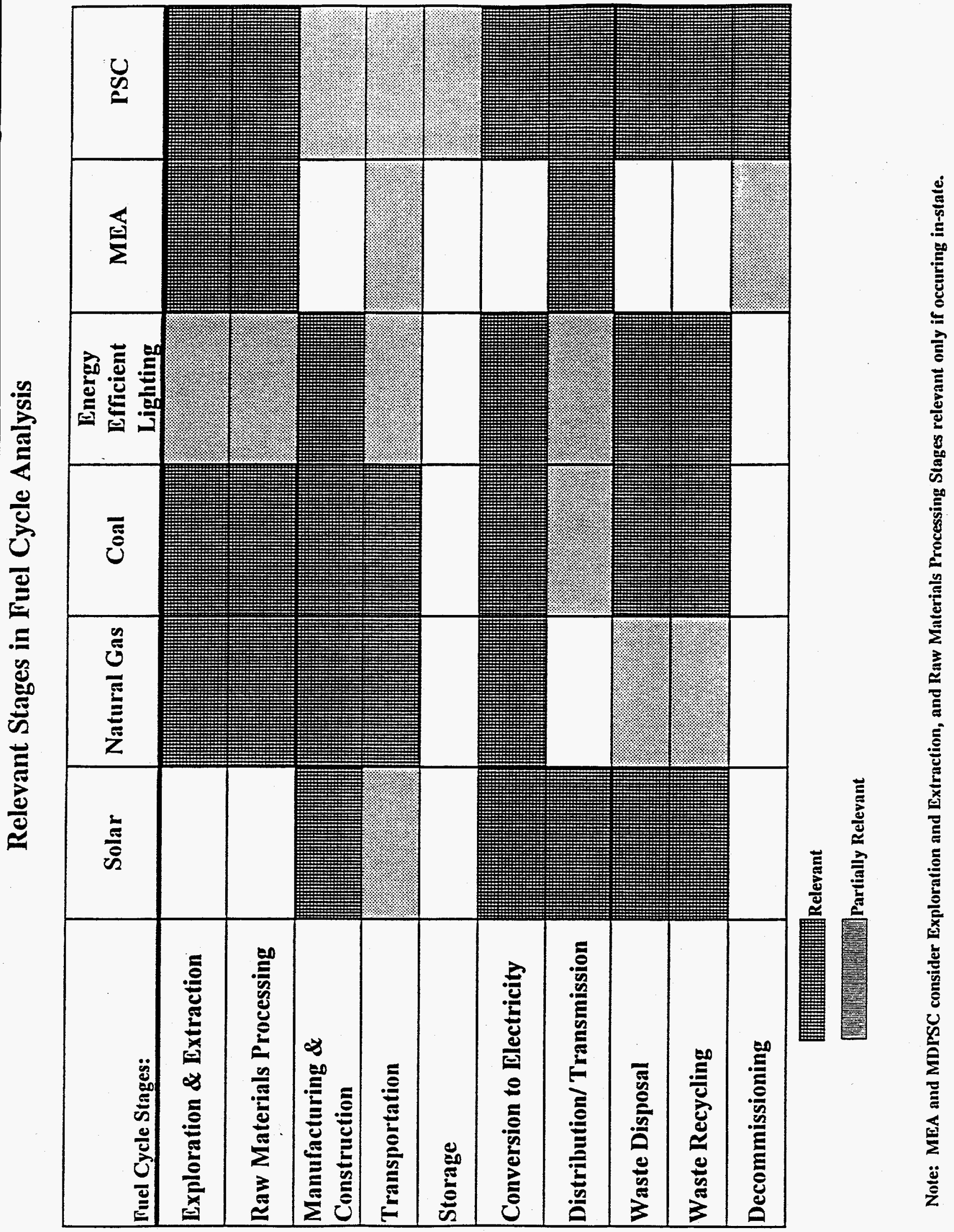




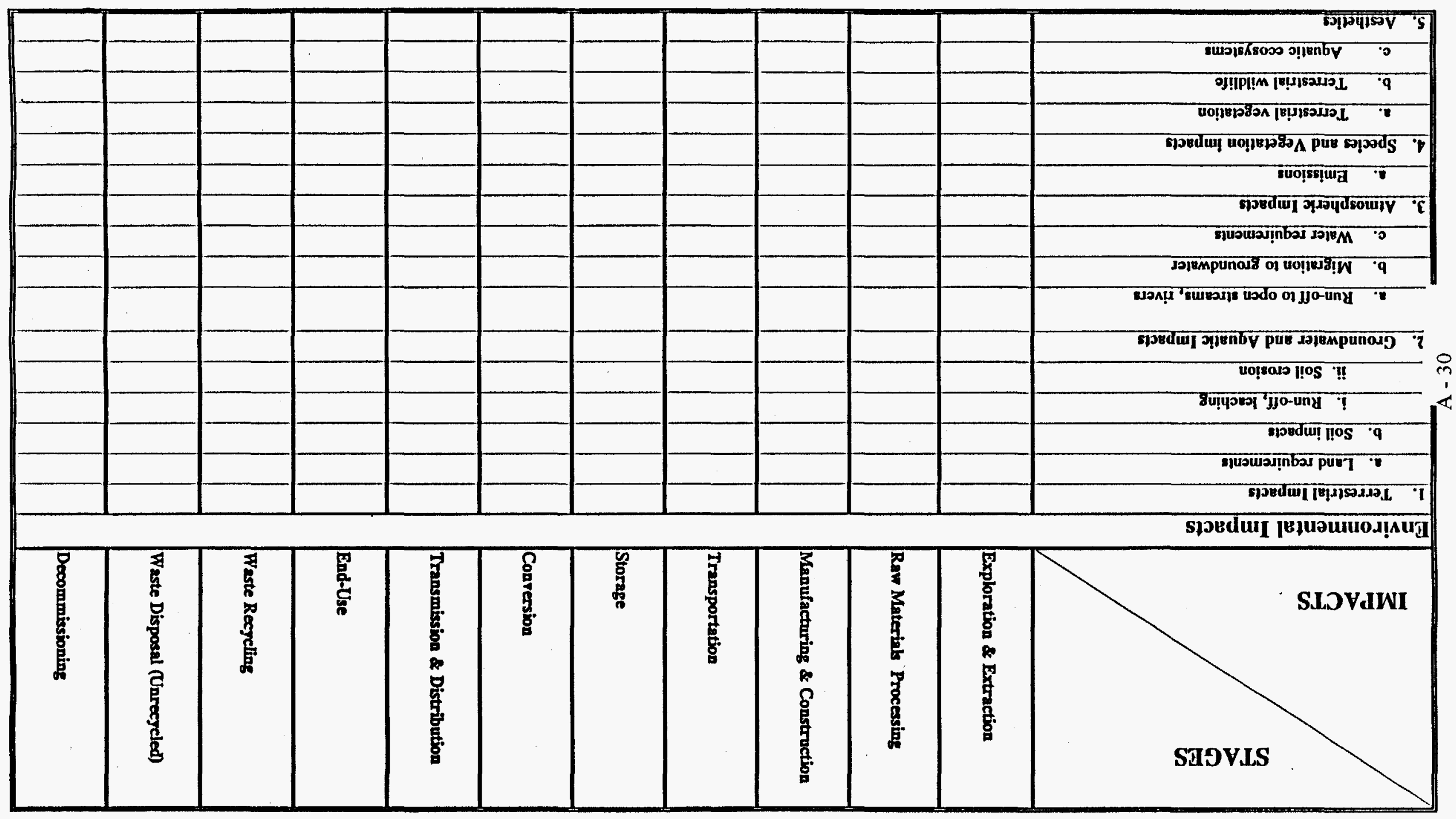

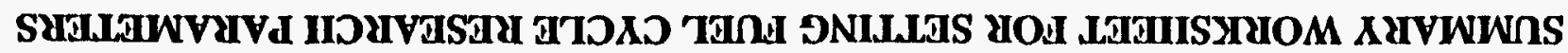




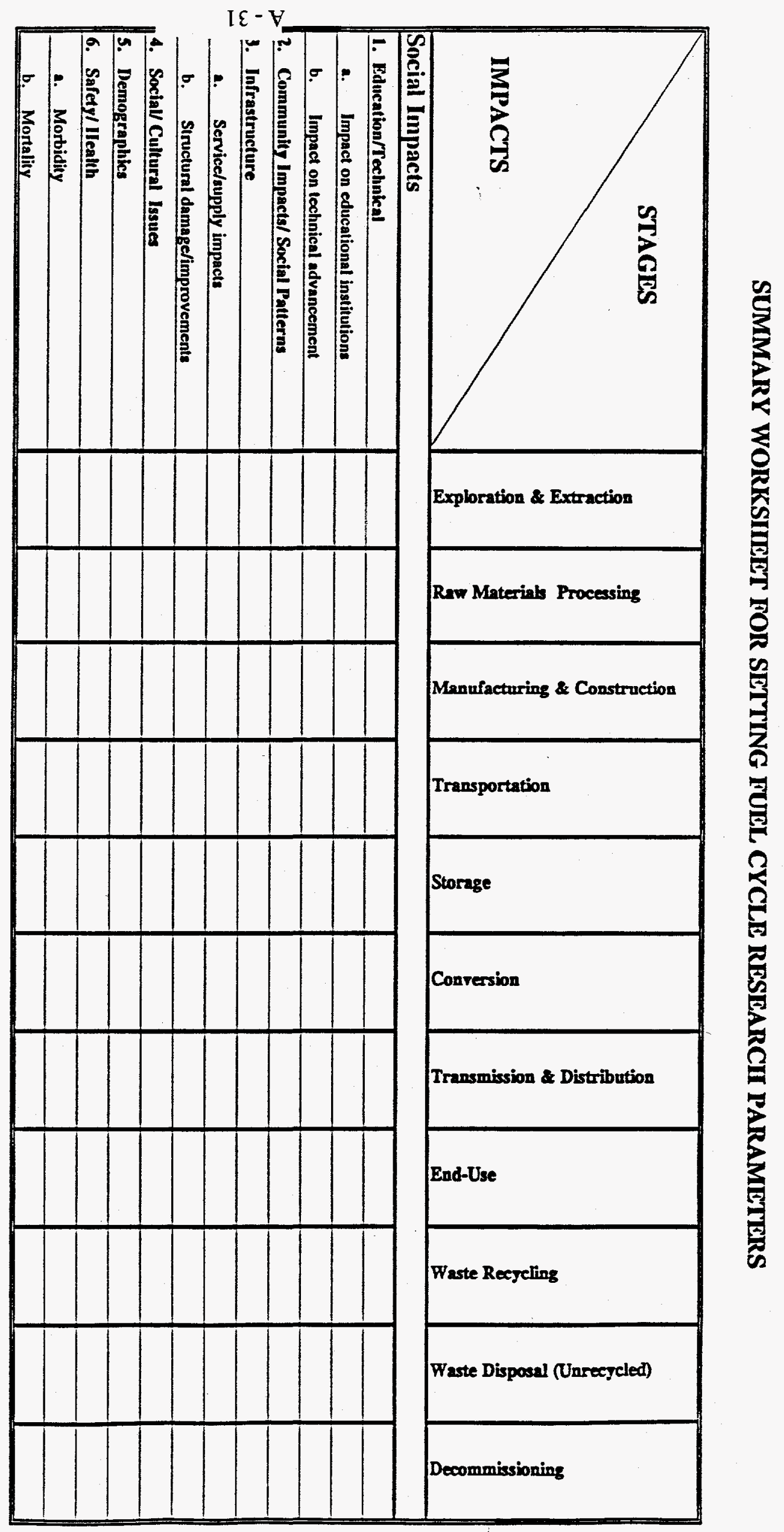


SUMMARY WORKSIIEET FOR SETTING FUEL CYCLE RESEARCII PARAMETERS

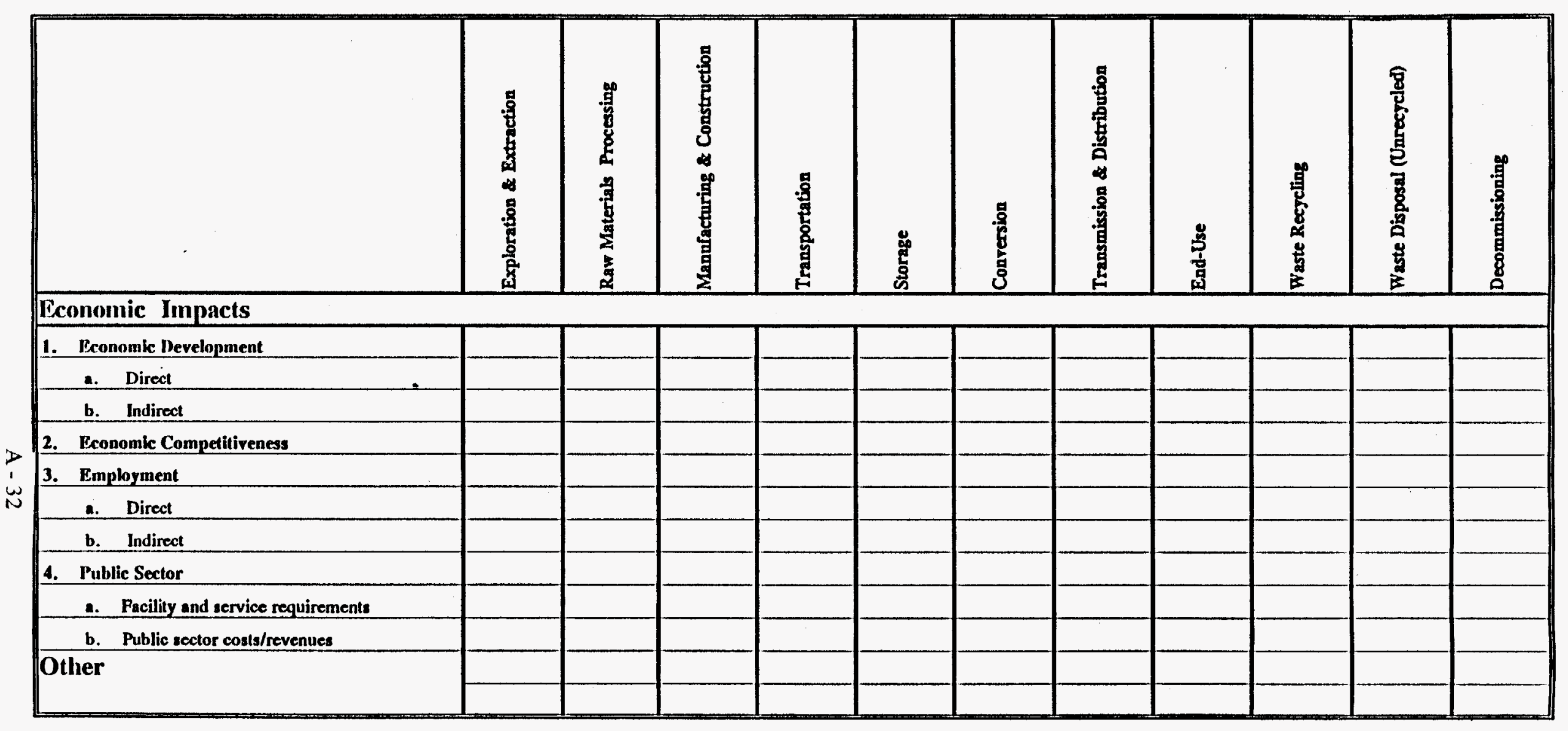




\section{RELEVANT IMPACTS IN FUEL CYCLE ANALYSIS}

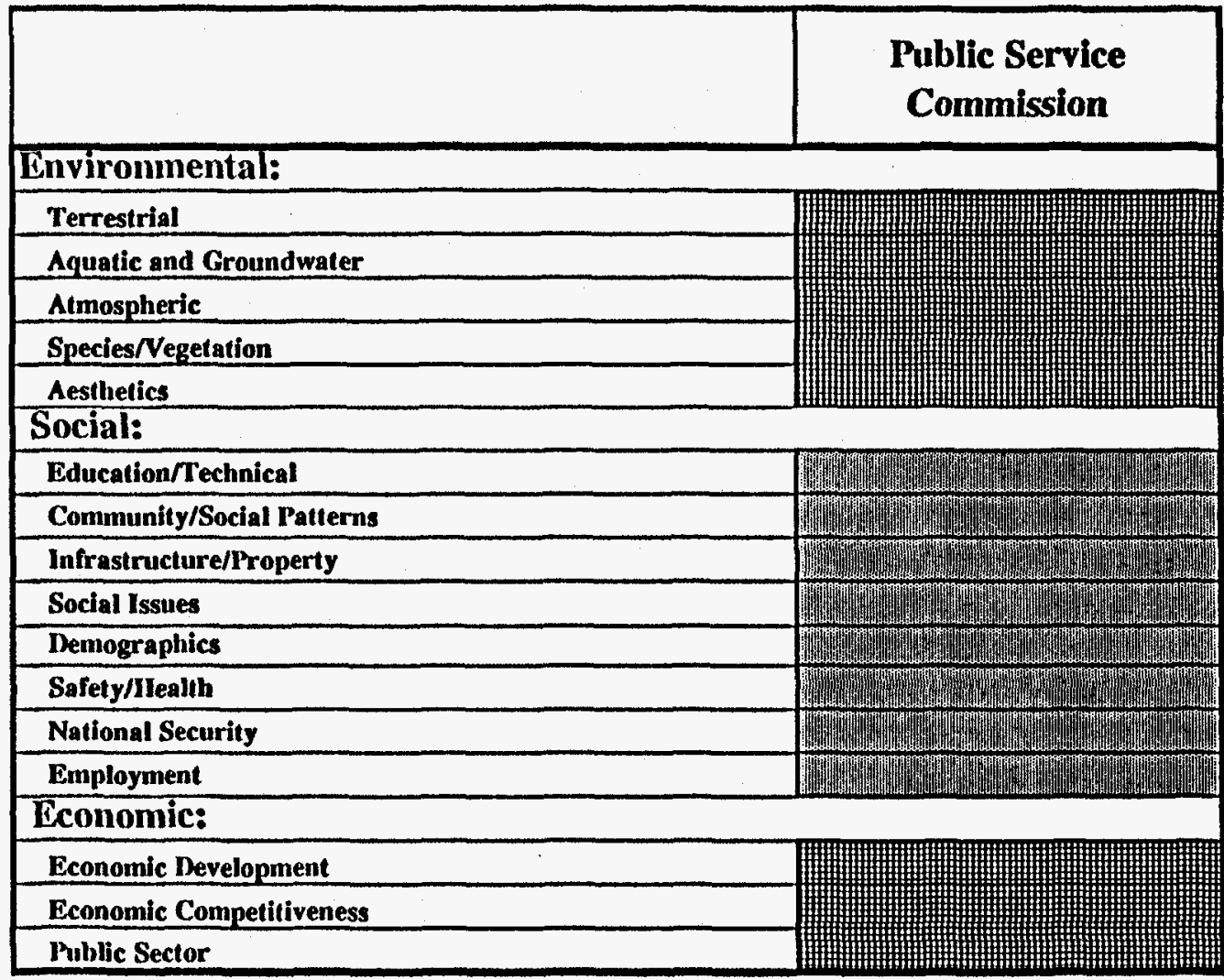

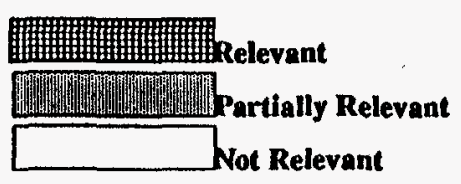

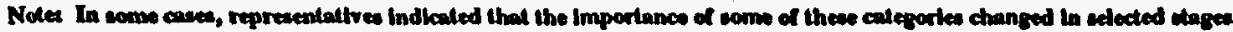


STAGE 1: EXPLORATION \& EXTRACTION - The identification and development of primary energy resources. This includes impacts associated with extracting raw materials such as coal, natural gas, mercury, or harvesting timber or bio-crops. Impacts directly associated with extraction of primary resources are considered relevant. The analysis does not include impacts associated with co-product derelopment.

\begin{tabular}{|c|c|c|c|}
\hline A. ENVIRONMENTAL IMPACTS & $\begin{array}{l}\text { Include } \\
\text { (YN } \mathbf{N} \text { ) }\end{array}$ & $\begin{array}{l}\text { Qualitative } \\
\text { Measure }\end{array}$ & $\begin{array}{c}\text { Quantitative } \\
\text { Measure }\end{array}$ \\
\hline \multicolumn{4}{|c|}{ 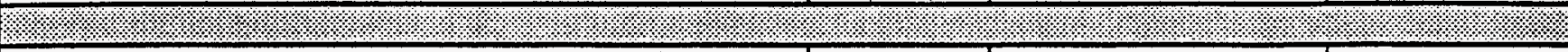 } \\
\hline \multicolumn{4}{|l|}{ 1. Terrestrial Impacts } \\
\hline \multicolumn{4}{|l|}{ a. Land requirements } \\
\hline i. Mining & & & \\
\hline- coal & & & \\
\hline - mercury & & & \\
\hline \multicolumn{4}{|l|}{-} \\
\hline \multicolumn{4}{|l|}{ - } \\
\hline \multicolumn{4}{|l|}{ ii. Drilling: } \\
\hline \multicolumn{4}{|l|}{ - oil } \\
\hline \multicolumn{4}{|l|}{$-\quad$ gas } \\
\hline \multicolumn{4}{|l|}{-} \\
\hline \multicolumn{4}{|l|}{ iii. $\quad$ Rehabilitation of existing sites } \\
\hline \multicolumn{4}{|l|}{ iii. Rehabilitation of existing sites } \\
\hline \multicolumn{4}{|l|}{$\begin{array}{ll}- & \text { reforestation } \\
- & \text { land reclamation }\end{array}$} \\
\hline - land reclamation & & & \\
\hline \multicolumn{4}{|l|}{-} \\
\hline \multicolumn{4}{|l|}{ - } \\
\hline \multicolumn{4}{|l|}{ b. Soil impacts } \\
\hline \multicolumn{4}{|l|}{ i. Runoff, solute migration } \\
\hline \multicolumn{4}{|l|}{$\begin{array}{ll}- & \text { metals } \\
- & \text { tailings }\end{array}$} \\
\hline - tailings & & & \\
\hline \multicolumn{4}{|l|}{ tailings } \\
\hline \multicolumn{4}{|l|}{-} \\
\hline \multicolumn{4}{|l|}{ ii. Soil erosion (i.e. logging, mining) } \\
\hline \multicolumn{4}{|l|}{ dissolved solids } \\
\hline \multicolumn{4}{|l|}{ - turbidity } \\
\hline \multicolumn{4}{|l|}{-} \\
\hline \multicolumn{4}{|l|}{$\cdot$} \\
\hline \multicolumn{4}{|l|}{ c. Other } \\
\hline \multicolumn{4}{|l|}{ 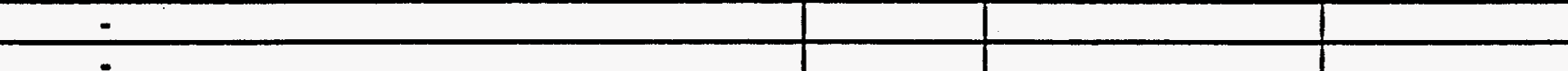 } \\
\hline- & & & \\
\hline \multicolumn{4}{|l|}{ 2. Groundwater impacts } \\
\hline \multicolumn{4}{|l|}{$\begin{array}{l}\text { a. Leaching, rumoff, migration of } \\
\text { constituents to the aquifer }\end{array}$} \\
\hline- tailings & & & \\
\hline - brine production & & & \\
\hline- & & & \\
\hline- & & & \\
\hline
\end{tabular}




\section{FUEL CYCLE DATA RESEARCH}

- BCS RESEARCHED FUEL CYCLE DATA FOR EACH OF THE FOUR CASE APPLICATIONS USING THE BOUNDARIES ESTABLISHED BY THE WORKING GROUP

- DATA RESOURCES VARIED SIGNIFICANTLY BY STAGE, TYPE OF IMPACT, AND TYPE OF APPLICATION OR RESOURCE BEING EVALUATED 


\section{Comparison of Resources and Gaps in Fuel Cycle Data}

\begin{tabular}{|c|c|c|c|c|}
\hline Fuel Cycle Stages & Natural Gas & Coal & Lighting & Photovoltaics \\
\hline \multicolumn{5}{|c|}{ Exploration \& Extraction } \\
\hline \multirow{3}{*}{\begin{tabular}{|l|} 
Environment \\
Social \\
Economic \\
\end{tabular}} & & Nin & & \\
\hline & & SPEOA & & \\
\hline & & 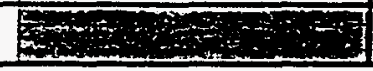 & & \\
\hline \multicolumn{5}{|c|}{ Raw Materials Processing } \\
\hline \multirow{3}{*}{\begin{tabular}{|l|} 
Environment \\
Social \\
Economic \\
\end{tabular}} & & & & \\
\hline & $\because$ & $5=2,23$ & & \\
\hline & & 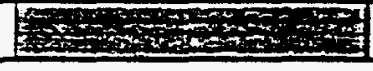 & & \\
\hline \multicolumn{5}{|c|}{ Manufacturing \& Construction } \\
\hline \multirow{3}{*}{\begin{tabular}{|l|} 
Environment \\
Social \\
Economic \\
\end{tabular}} & \multicolumn{2}{|c|}{ 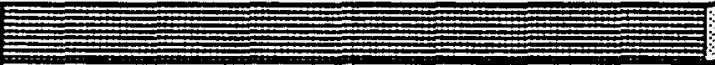 } & 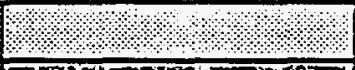 & 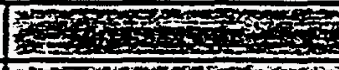 \\
\hline & \multicolumn{2}{|c|}{ 帒 } & 75y & 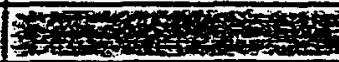 \\
\hline & \multicolumn{2}{|c|}{ X3 } & & 5 \\
\hline \multicolumn{5}{|l|}{ Transportation } \\
\hline Environment & \multicolumn{2}{|c|}{35 3 } & $9.1 \%$ & \\
\hline Social & \multicolumn{2}{|c|}{ 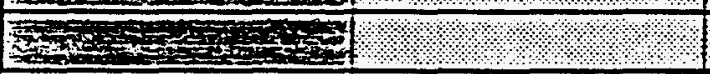 } & 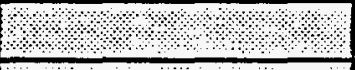 & \\
\hline Economic & \multicolumn{2}{|c|}{ 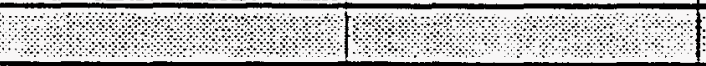 } & 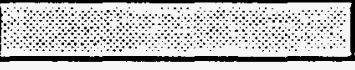 & \\
\hline \multicolumn{5}{|l|}{ Storage } \\
\hline \multicolumn{5}{|l|}{ Environment } \\
\hline \multicolumn{5}{|l|}{ Social } \\
\hline Economic & & & & \\
\hline
\end{tabular}

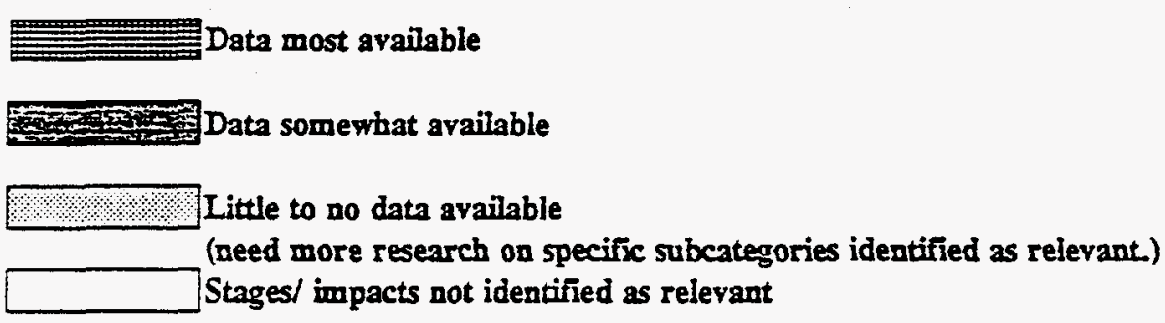

1. Stage nod in analysis because conl in the case exmple is not produced in MD, howerer, MRA \& PSC said more information on conl impects would be beipfal.

2. For Gas \& Coal technologies, impects are primanily construction related. With ropect to Lighting \& PV, impacts are reiated to the manufacturing phase. 
Comparison of Resources and Gaps in Fuel Cycle Data

\begin{tabular}{|c|c|c|c|c|}
\hline Fuel Cycle Stages & Natural Gas & Coal & Lighting & Photoroltaics \\
\hline \multicolumn{5}{|c|}{ Conversion to Electricity } \\
\hline \multicolumn{5}{|l|}{ Environment } \\
\hline Social & \multicolumn{3}{|c|}{ 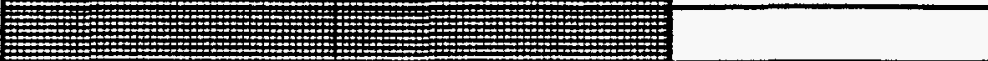 } & BWSOS \\
\hline Economic & \multicolumn{3}{|c|}{ - } & 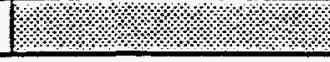 \\
\hline \multicolumn{5}{|c|}{ Distribution/ Transmission } \\
\hline \multicolumn{5}{|l|}{ Environment } \\
\hline \multicolumn{5}{|l|}{ Social } \\
\hline \\
\hline \multicolumn{5}{|l|}{ Waste Disposal } \\
\hline \multicolumn{5}{|c|}{ 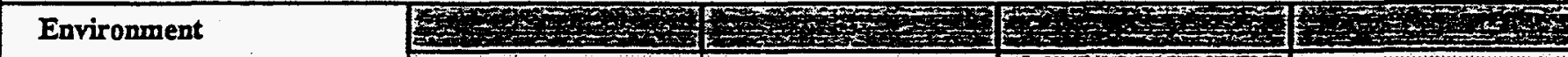 } \\
\hline \multicolumn{5}{|c|}{ 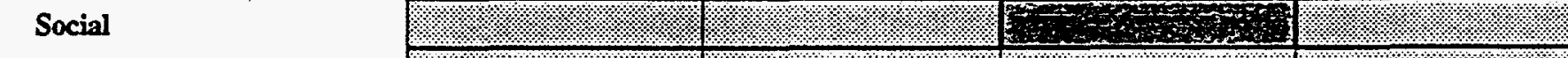 } \\
\hline Economic & 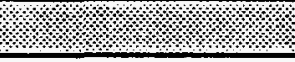 & 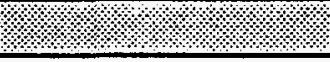 & $\sqrt[3]{2}$ & 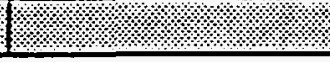 \\
\hline \multicolumn{5}{|l|}{ Waste Recycling } \\
\hline \multicolumn{5}{|c|}{ 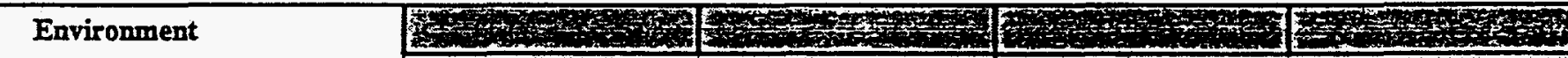 } \\
\hline \multicolumn{5}{|c|}{ 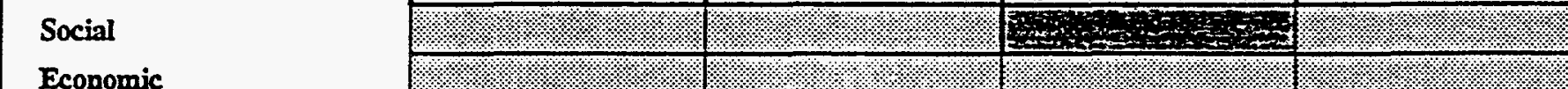 } \\
\hline Economic & ? & 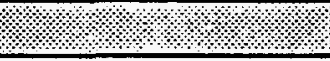 & IIn: & 1 \\
\hline \multicolumn{5}{|l|}{ Decommissioning } \\
\hline \multicolumn{5}{|l|}{ Environment } \\
\hline \multicolumn{5}{|l|}{ Social } \\
\hline Economic & & & & \\
\hline
\end{tabular}

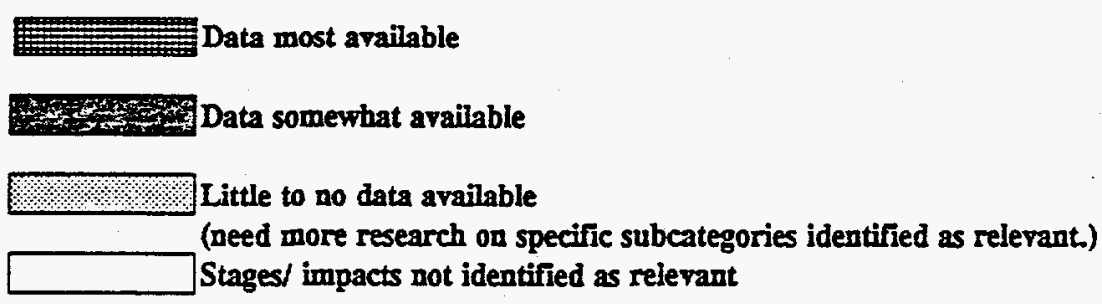




\section{REVISIONS TO THE GUIDE}

- ADDED SUMMARY APPLICATION WORKSHEETS

- STREAMLINED THE DETAILED FUEL CYCLE WORKSHEETS

- ADDED AN APPENDIX OF POTENTIAL DATA AND ANALYTICAL TOOLS

- PUT WORKSHEETS INTO AN EXCEL V4.0 SPREADSHEET FORMAT 


\section{OBSERVATIONS}

- OPPORTUNITIES TO IMPROVE THE INFORMATION AVAILABLE FOR PLANNING PROCESSES SHOULD BE PURSUED WHEN COST-EFFECTIVE

- THE GUIDE IS AN EFFECTIVE AND STREAMLINED TOOL FOR FRAMING FCA

- $\quad$ Covers a Broad Range of Potential Costs/Benefits Allowing the Working Group to Identify Costs/Benefits of Key Concern

- Can Account for Temporal and Geographic Boundaries

- Does not Restrict Analysis to Simply Environmental Externalities

- $\quad$ THE GUIDE COULD BE A USEFUL TOOL TO AUGMENT:

- Analyzing Selected Resources/Applications in IRP

- $\quad$ Site Evaluations

- State Energy Strategy Development

- Utility Strategic Planning

- R\&D Program Screening and Prioritization

- THE GUIDE IS EASILY ADAPTED TO IRP PROCESSES 


\section{OBSERVATIONS (Cont'd.)}

- TFCA AND OTHER FORMS OF LIFE CYCLE ANALYSIS CAN BE EFFECTIVE METHODS FOR IDENTIFYING OPPORTUNITIES TO INCREASE EFFICIENCY AND REDUCE ADVERSE SOCIETAL, ECONOMIC AND ENVIRONMENTAL IMPACTS

- IT IS IMPORTANT TO EVALUATE IMPACTS BOTH QUANTITATIVELY AND QUALITATIVELY

- DATA AVAILABILITY AND QUALITY REMAINS A CRITICAL BARRIER 


\title{
ASPEN as a Total Fuel Cycle Modeling Tool
}

\author{
Stephen Adelman \\ National Renewable Energy Laboratory \\ Austin, Texas \\ October 6, 1994 \\ Total Fuel Cycle Assessment Workshop
}

\section{NREL TFC Modeling Requirements}

- NREL needed the ability to efficiently perform TFC analysis

- Take advantage of existing in-house databases

- Use existing software

- Have the capability to rapidly describe large utility systems and component processes

- Solution: ASPEN + ORACLE + customization

- ASPEN (Advanced Simulator for Process Engineering (ASPEN Tech., Inc.): system and component modeling

- ORACLE: data engine

- Customization: connectivity, dispatch modeling 


\section{NREL TFC Modeling Requirements (Continued)}

Utility dispatch modeling

- Probabilistic algorithm - method of moments and cumulents (annual or seasonal)

- Hourly algorithms - linear and non-linear constrained minimization

- Economic and emission-constrained dispatch

- Dispatch algorithm data requirements

- Hourly loads, sales, purchases, DSM

- Unit characteristics: capacities, heat rates, FOR's, production costs, emission rates

- Maintenance schedules

\section{Software Components}

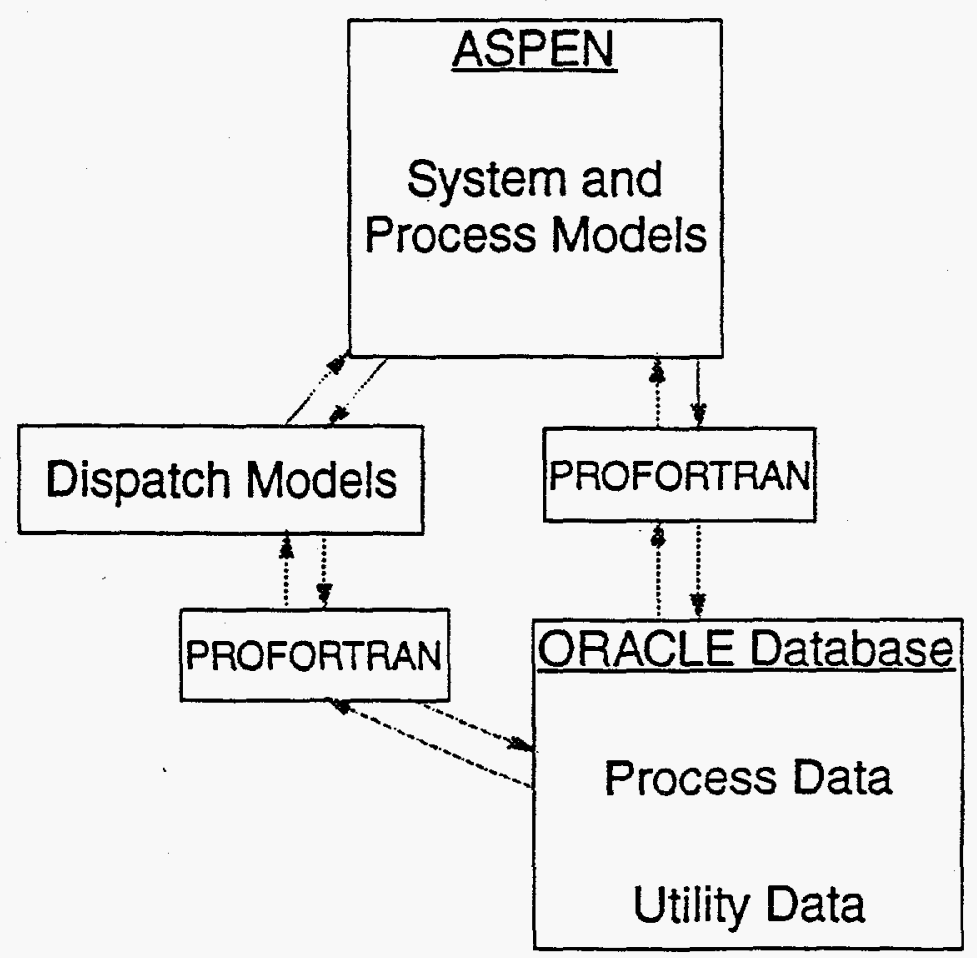




\section{Application to EVTECA}

" EVTECA: Electric Vehicle Total Energy Cycle Analysis

- DOE ( $E E, N E$ ) comparitive emissions study ( $E V$ vs RFG in 2000 and 2010)

- Los Angeles (SCE, LADWP)

- Chicago (Commonwealth Edison)

- Houston (HL\&P)

- Washington, D.C. NEPCO, PEPCO, BG\&E)

- In addition to other tasks, NREL is performing the utility analysis

Utility Simulation

Data aquisition, analysis, and preparation

- Hourly loads and load growth projections, sales, purchases, DSM

- Generation characteristics: unit capacities, heat rates,

FOR's, production costs, emission rates

- Capacity expansion plans, maintenance schedules

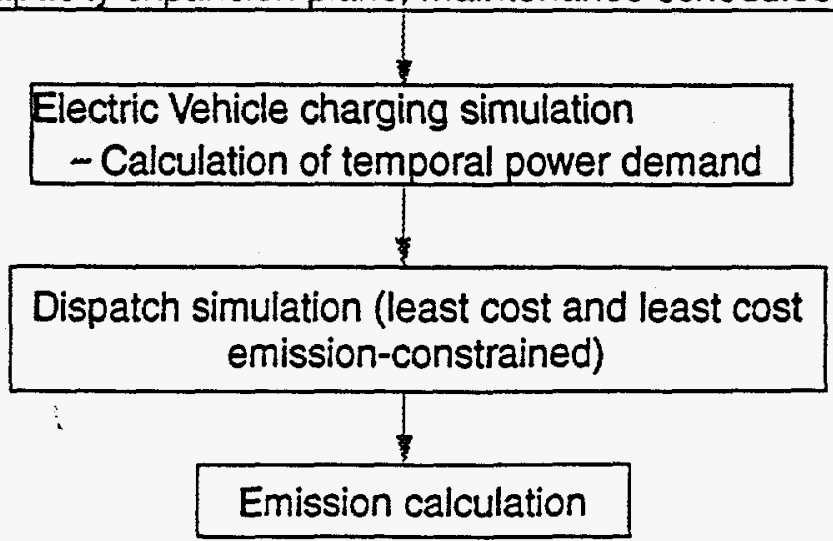

al Fuel Cycle Assessment Workshop - October 6, 1994 A - 43 


\section{Summary}

ASPEN: powerful, flexible

- Readily adaptable for TFC modeling with addition of other software components

- Future Applications

- H2 storage in an electric utility

- Effect on cost and emissions 


\title{
Think Globally, Calculate Locally
}

\section{The American Gas Cooling Center Resource Energy Efficiency and Emissions Database and Modeling Program}

\author{
Michael J. Witte, Ph.D. \\ ElectroCom GARD, Niles, Illinois \\ Kenneth M. Green \\ BCS, Incorporated, Columbia, Maryland \\ Richard S. Sweetser \\ American Gas Cooling Center, Arlington, Virginia \\ DOE Total Fuel Cycle Assessment Workshop \\ October 6-7, 1994, Austin, Texas
}




\section{Objective}

Develop a database and model to estimate the seasonal resource energy efficiency and related emissions in delivering natural gas and electricity to end-use equipment. 


\section{Motivation}

- Commonly used national annual average resource energy efficiencies

$$
\begin{array}{ll}
\text { Electricity: } & 33 \% \\
\text { Gas: } & 91 \%
\end{array}
$$

- Are these right for space conditioning electricity?

- Is there seasonal variation?

- Is there variation among utilities?

- What about emissions? 

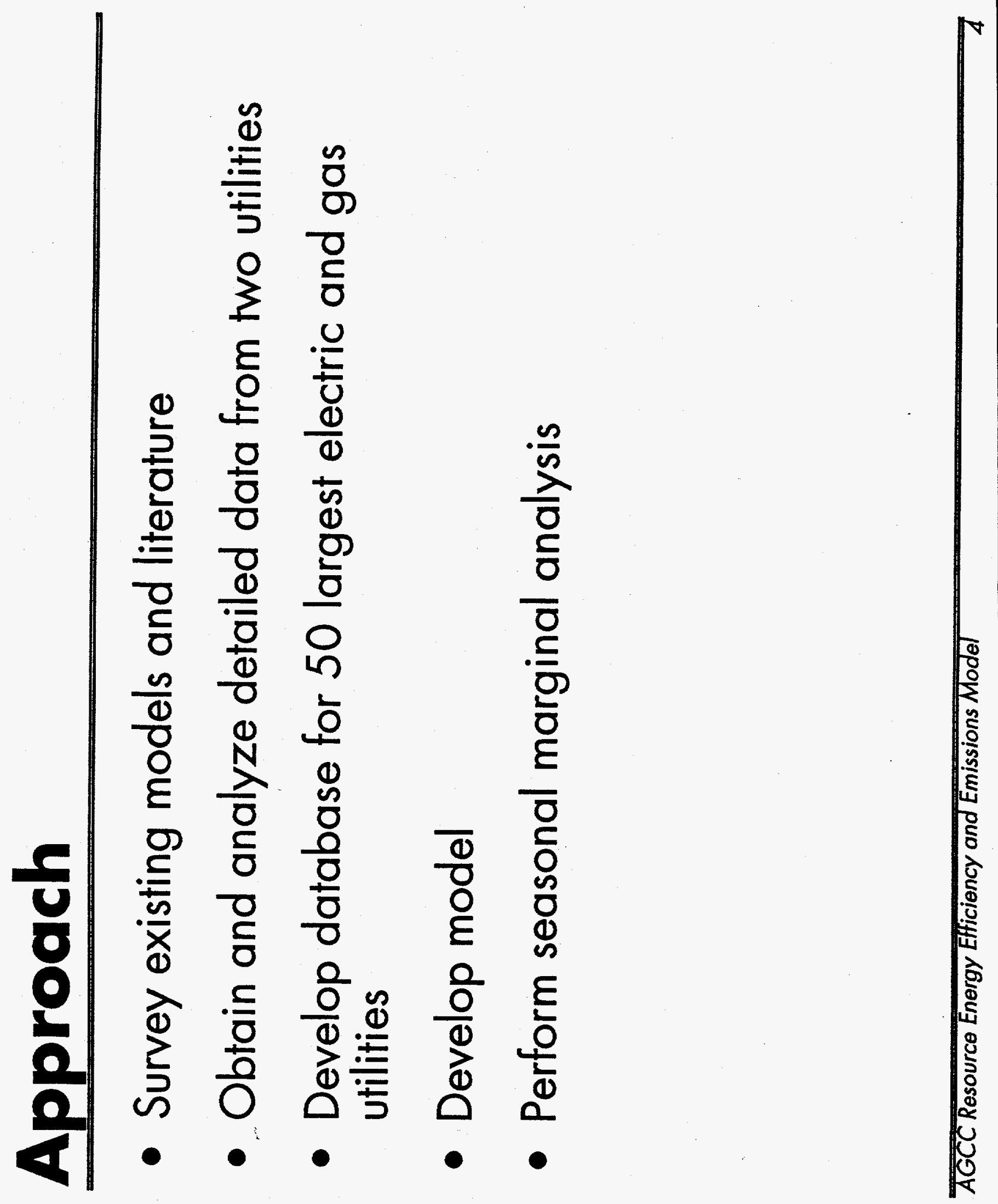


\section{Desired Model Characteristics}

- Open, non-proprietary methodology

- Based on publicly available data

- Sensitive to seasonal variations

Electric generation mix

Power plant efficiencies

T\&D losses

Gas pipeline compressor efficiencies

- Include upstream fuel cycle energy and emissions for fuel extraction, processing, and transportation

- Easy to use, spreadsheet environment 


\section{Database Development}

- Readily available monthly and annual data in electronic form

FERC-1, FERC-11

EIA-759, EIA-176, EIA-130, EIA-826

- Power generation emissions factors

- Upstream fuel cycle energy and emissions factors 


\section{Energy Supply Model Overview}
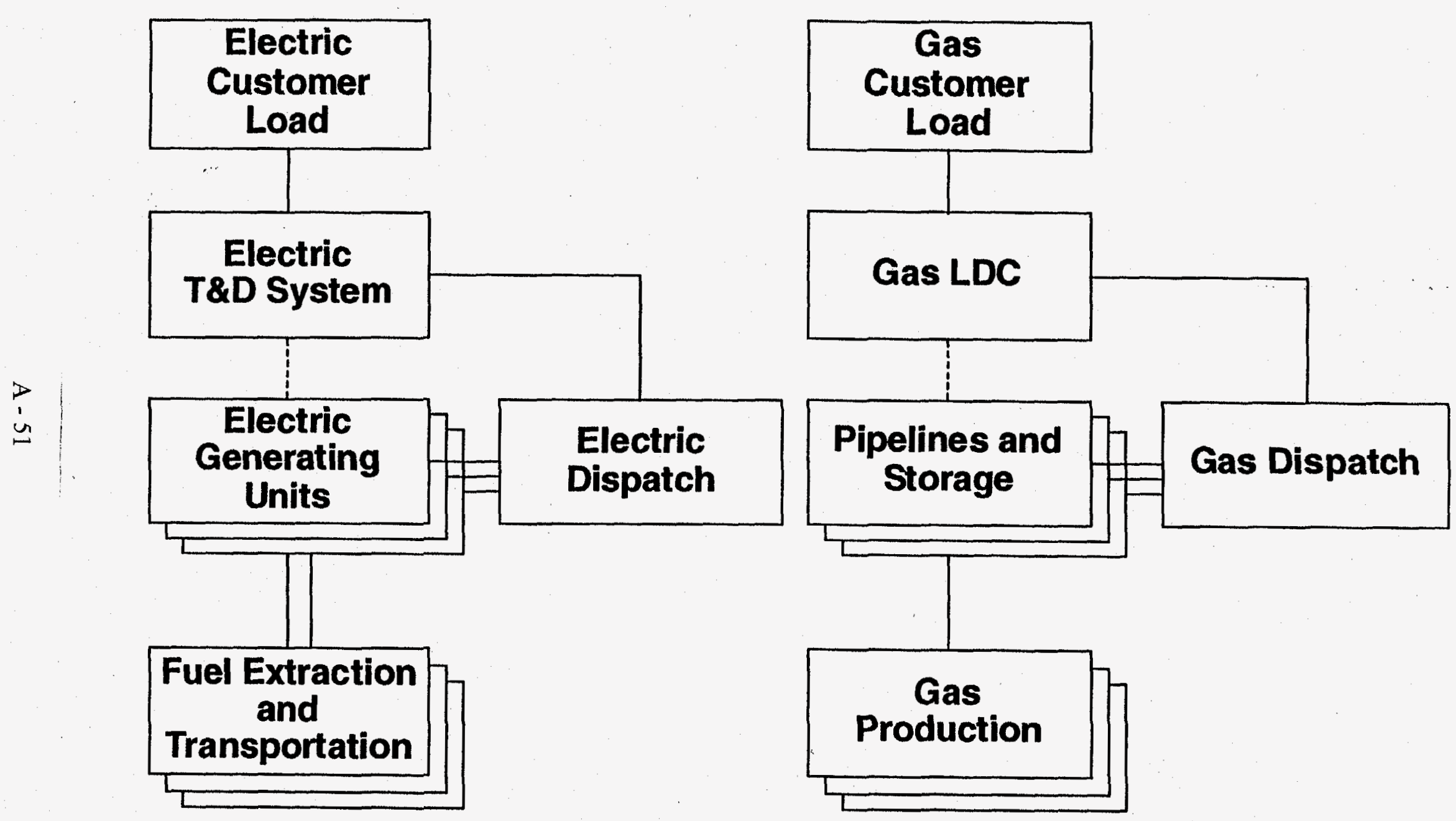

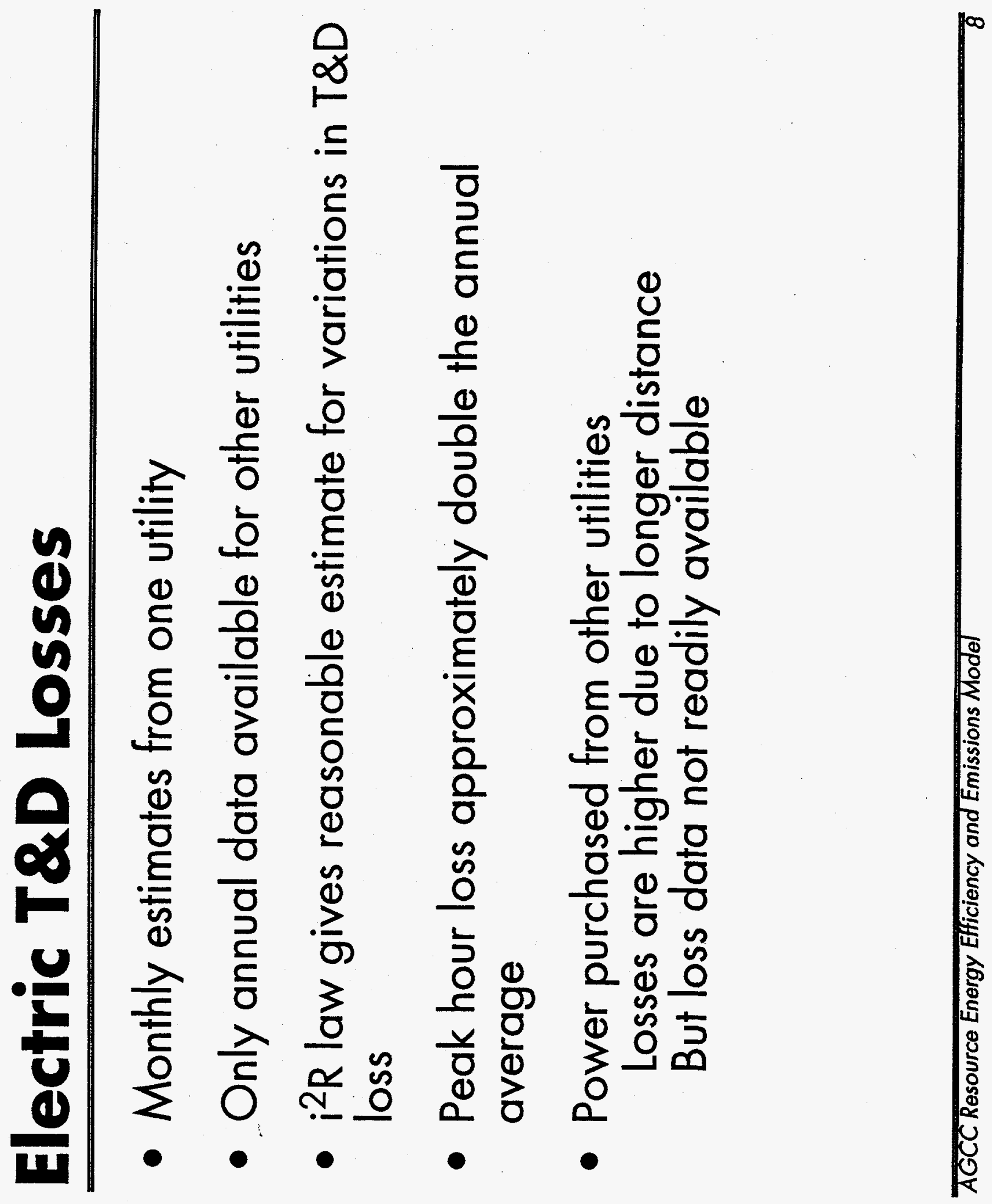


\section{Seasonal Marginal Analysis}

- Monthly data for each generating unit

- Assign generating units to classes: baseload, intermediate, or peak

- Determine seasonal efficiency and emissions factors per delivered $k W h$ for each class

- Space cooling is met by intermediate and peak generation

- Include all intermediate and peak generation for conservative estimate 


\section{Results for Detailed Analysis}

\begin{tabular}{|c|c|c|c|c|}
\hline Utility & $\begin{array}{c}\text { Resource } \\
\text { Energy } \\
\text { Efficiency }\end{array}$ & $\begin{array}{c}\mathrm{CO}_{2} \\
(\mathrm{lb} / \mathrm{kWh})\end{array}$ & $\begin{array}{c}\mathrm{NO}_{\mathrm{x}} \\
(\mathrm{lb} / \mathrm{kWh})\end{array}$ & $\begin{array}{c}\mathrm{SO}_{2} \\
(\mathrm{lb} / \mathrm{kWh})\end{array}$ \\
\hline $\begin{array}{l}\text { Cooling Electricity } \\
\text { Northeast } \\
\text { Mid-Atlantic } \\
\end{array}$ & $\begin{array}{l}23 \% \\
18 \% \\
\end{array}$ & $\begin{array}{l}2.1 \\
2.6 \\
\end{array}$ & $\begin{array}{l}0.004 \\
0.010 \\
\end{array}$ & \\
\hline $\begin{array}{l}\text { Electricity } \\
\text { ortheast } \\
\text { id-Atlantic }\end{array}$ & $\begin{array}{l}27 \% \\
30 \% \\
\end{array}$ & $\begin{array}{l}1.2 \\
1.2 \\
\end{array}$ & $\begin{array}{l}0.003 \\
0.004 \\
\end{array}$ & $\begin{array}{l}0.001 \\
0.008\end{array}$ \\
\hline
\end{tabular}




\section{Results for 52 Utilities}

- 52 largest utilities representing approximately $50 \%$ of U.S. utility generation

- Six years of data to average out unusual weather

\begin{tabular}{|c|c|c|c|c|}
\hline & $\begin{array}{l}\text { Resource } \\
\text { Energy } \\
\text { Efficiency }\end{array}$ & $\begin{array}{c}\mathrm{CO}_{2} \\
(\mathrm{lb} / \mathrm{kWh})\end{array}$ & $\begin{array}{c}N O_{x} \\
(\mathrm{lb} / \mathrm{kWh})\end{array}$ & $\begin{array}{c}\mathrm{SO}_{2} \\
(\mathrm{lb} / \mathrm{kWh})\end{array}$ \\
\hline $\begin{array}{l}\text { Cooling Electricity } \\
\text { Best } \\
\text { Average } \\
\text { Worst }\end{array}$ & $\begin{array}{l}32 \% \\
28 \% \\
22 \% \\
\end{array}$ & $\begin{array}{l}1.5 \\
2.1 \\
3.1\end{array}$ & $\begin{array}{l}0.005 \\
0.007 \\
0.011\end{array}$ & $\begin{array}{l}0.000 \\
0.013 \\
0.029\end{array}$ \\
\hline
\end{tabular}




\section{Resource Energy Efficiency}

\section{Space Cooling Electricity 1987-1992}

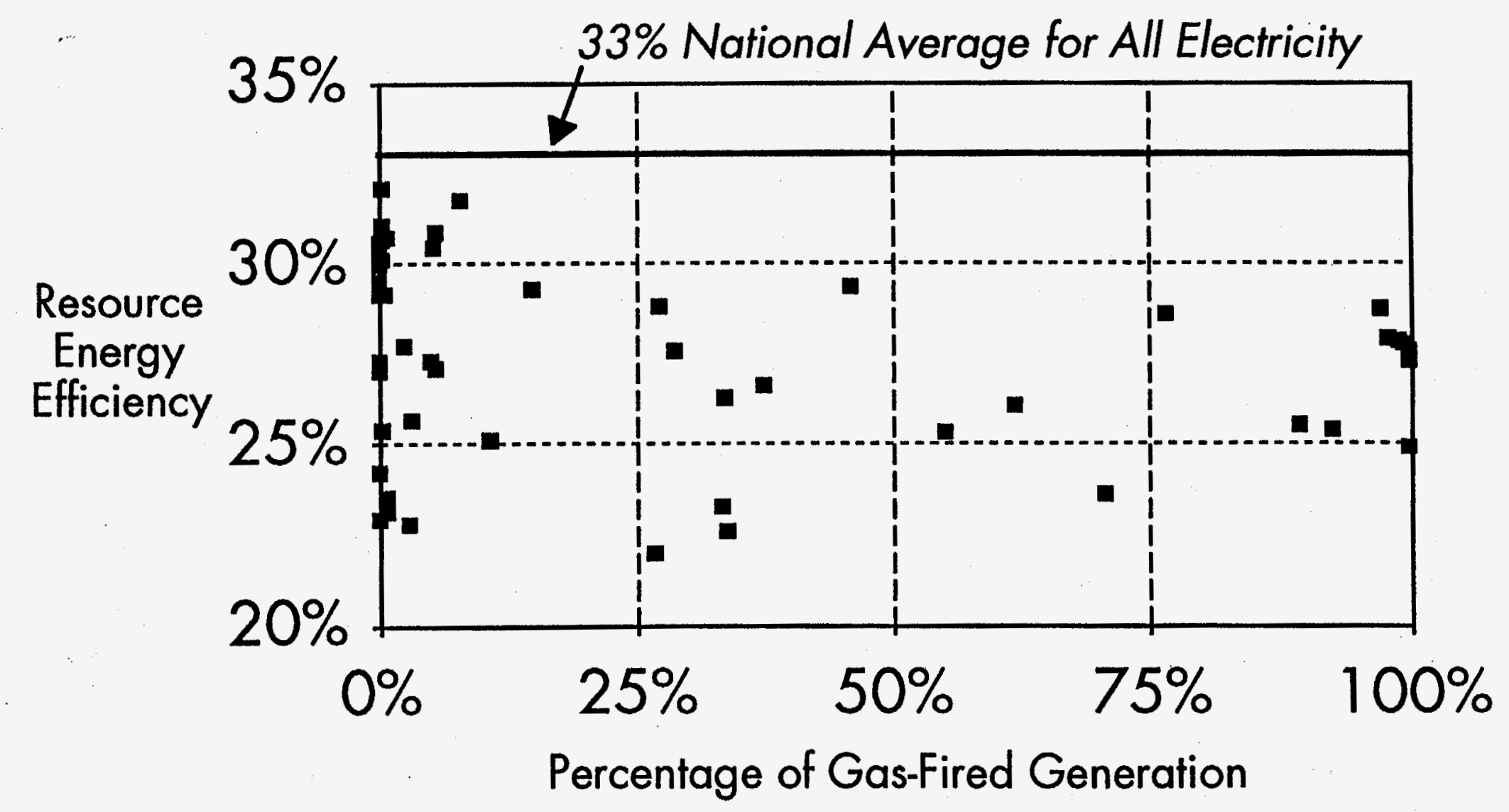




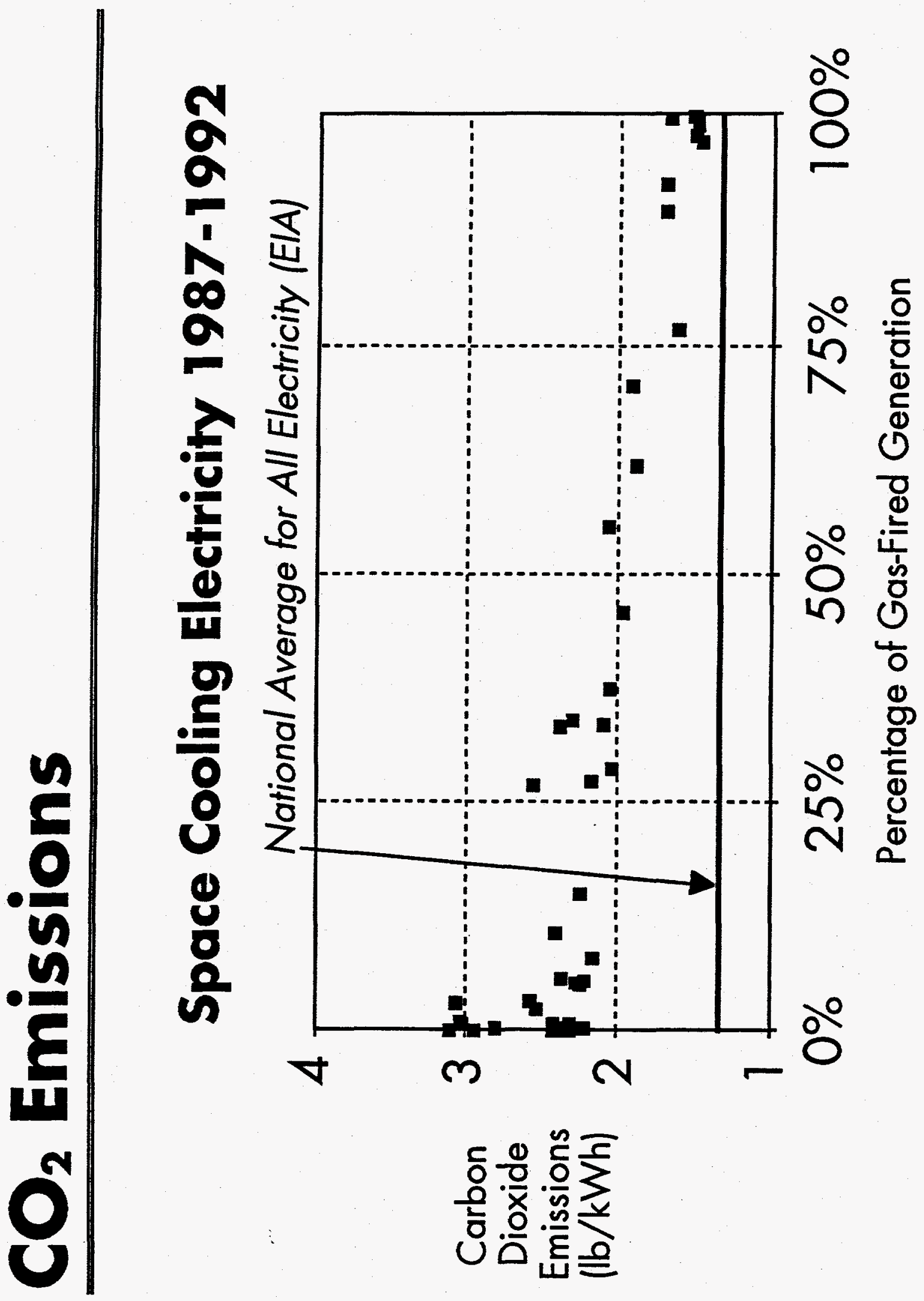




\section{Conclusions}

- Seasonal variations

- Generating efficiencies vary $2 \%$ or less between winter and summer

- Emissions vary for some utilities due to different fuel mixes

- Space cooling electricity resource energy efficiencies

- Range from $22 \%$ to $32 \%$

- Average $28 \%$

- All utilities below 33\% national average

- Space cooling electricity $\mathrm{CO}_{2}$ emissions

- Range from 1.5 to 3.1 (lb/kWh)

- Average 2.1

- All utilities well above 1.3 national average 


\section{Conclusions (cont'd)}

- National/regional annual average analysis is misleading for policy analysis for specific end uses

- To properly evaluate efficiency and emissions savings, analysis must be:

- Local

- Seasonal

- Marginal for a specific end use 


\section{Future Work}

- Gas system analysis

- Expanded electric utility database

- Purchased power and transmission loss database

- End-Use equipment comparisons 


\section{The New York \\ Environmental Externalities \\ Cost Study}

\section{Presentation at the \\ TOTAL FUEL CYCLE ASSESSMENT WORKSHOP \\ October 6-7, 1994 \\ Austin, Texas}

Robert D. Rowe

RCG/Hagler Bailly

1881 9th Street, Suite 201

Boulder, CO 80302

(303) 449-5515 


\section{- Clients/Management Board}

Empire State Electric Energy Research Corporation New York State Energy Research and Development Authority State of New York, Department of Public Service Electric Power Research Institute Resources for the Future (non-funding)

- Objectives:

- Develop a methodology and a user-friendly computer model to quantify physical and economic values for environmental impacts from electricity generation in New York

- Address all significant air, water, and land use impacts

- Scientifically defensible with uncertainty analysis

- Available for use in all regulatory decisions to license and relicense facilities 


\begin{tabular}{|c|c|}
\hline \multicolumn{2}{|c|}{ Electric Resource Options in EXMOD } \\
\hline Resource Type & Options \\
\hline Coal & $\begin{array}{l}\text { > Boiler using pulverized coal } \\
>\text { Atmospheric fluidized bed combustion (AFBC) } \\
>\text { Pressurized fluidized bed combustion (PFBC) } \\
\text { > Integrated gasified combined cycle (IGCC) }\end{array}$ \\
\hline Oil & $\begin{array}{l}>\text { Boiler using residual oil } \\
>\text { Combustion turbine (CT) using distillate oil } \\
>\text { Combined cycle (CC) using distillate oil }\end{array}$ \\
\hline Natural Gas & $\begin{array}{l}>\text { Combustion turbine }(\mathrm{CT}) \\
>\text { Combined cycle }(\mathrm{CC}) \\
>\text { Boiler }\end{array}$ \\
\hline Nuclear & $\begin{array}{l}>\text { Pressurized water reactor (PWR) } \\
>\text { Boiling water reactor (BWR) }\end{array}$ \\
\hline $\begin{array}{l}\text { Municipal Solid } \\
\text { Waste (MSW) }\end{array}$ & $>$ Boiler using refuse-derived fuel (RDF) \\
\hline Hydroelectric & $\begin{array}{l}>\text { Run-of-river (ROR) } \\
>\text { Pumped storage (PS) }\end{array}$ \\
\hline Biomass & $>$ Boiler using wood \\
\hline Wind & $>$ Central wind farm \\
\hline Solar & $\begin{array}{l}>\text { Central photovoltaic } \\
>\text { Central thermal }\end{array}$ \\
\hline $\begin{array}{l}\text { Demand Side } \\
\text { Management } \\
(\text { DSM)* }\end{array}$ & $\begin{array}{l}>\text { Efficient lighting } \\
>\text { Weatherization } \\
>\text { Efficient motors } \\
\text { > Appliance replacement }\end{array}$ \\
\hline $\begin{array}{r}\text { The } \\
\text { exte } \\
\text { are } \\
\text { RCG/Hagl }\end{array}$ & $\begin{array}{l}\text { irrent version of the externality model includes no } \\
\text { nality quantification for DSM programs. Zero damages } \\
\text { ssumed. } \\
\text { I Bailly, } 1994 \text {. }\end{array}$ \\
\hline
\end{tabular}


GEOGRAPHIC COVERAGE IN EXMOD

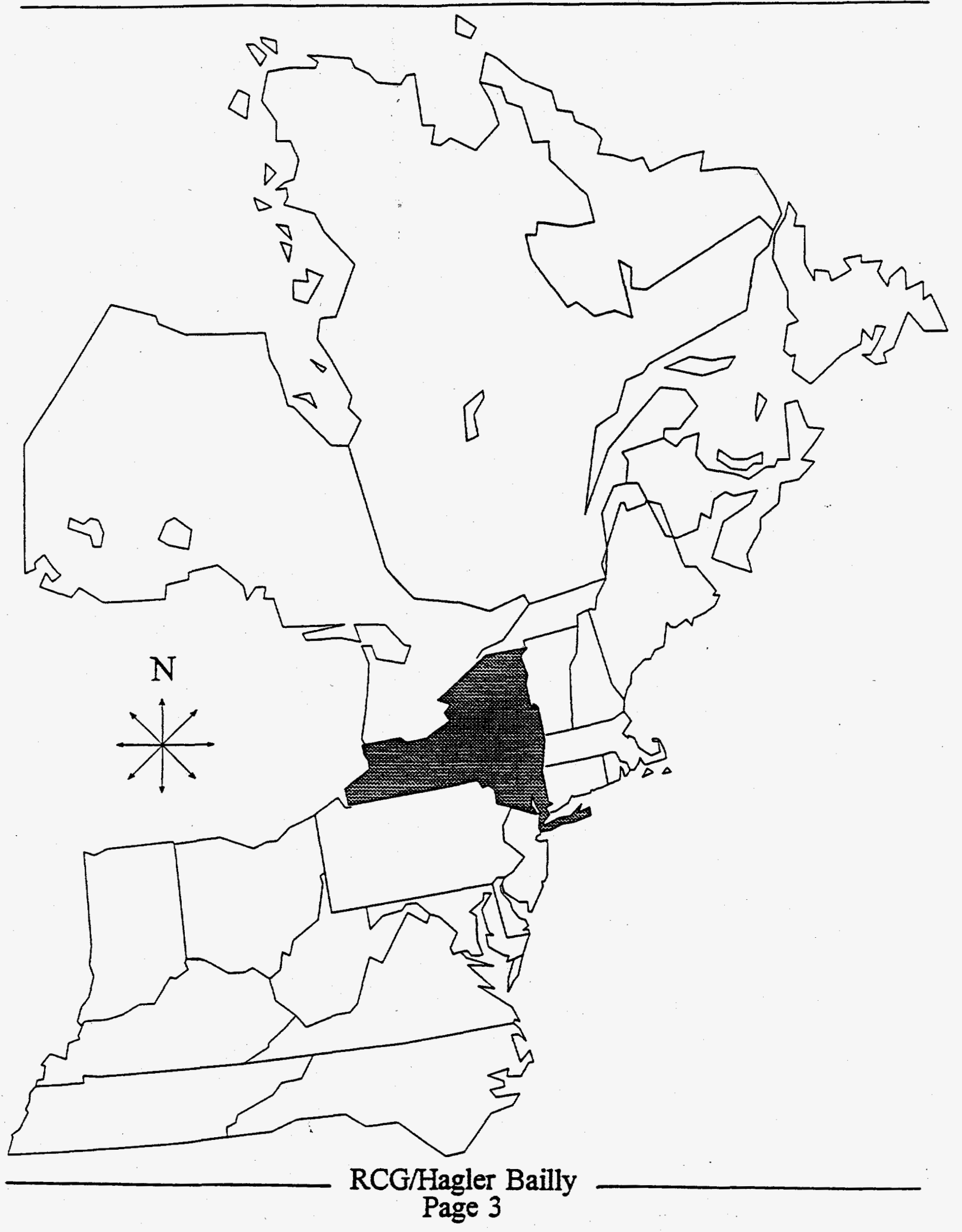




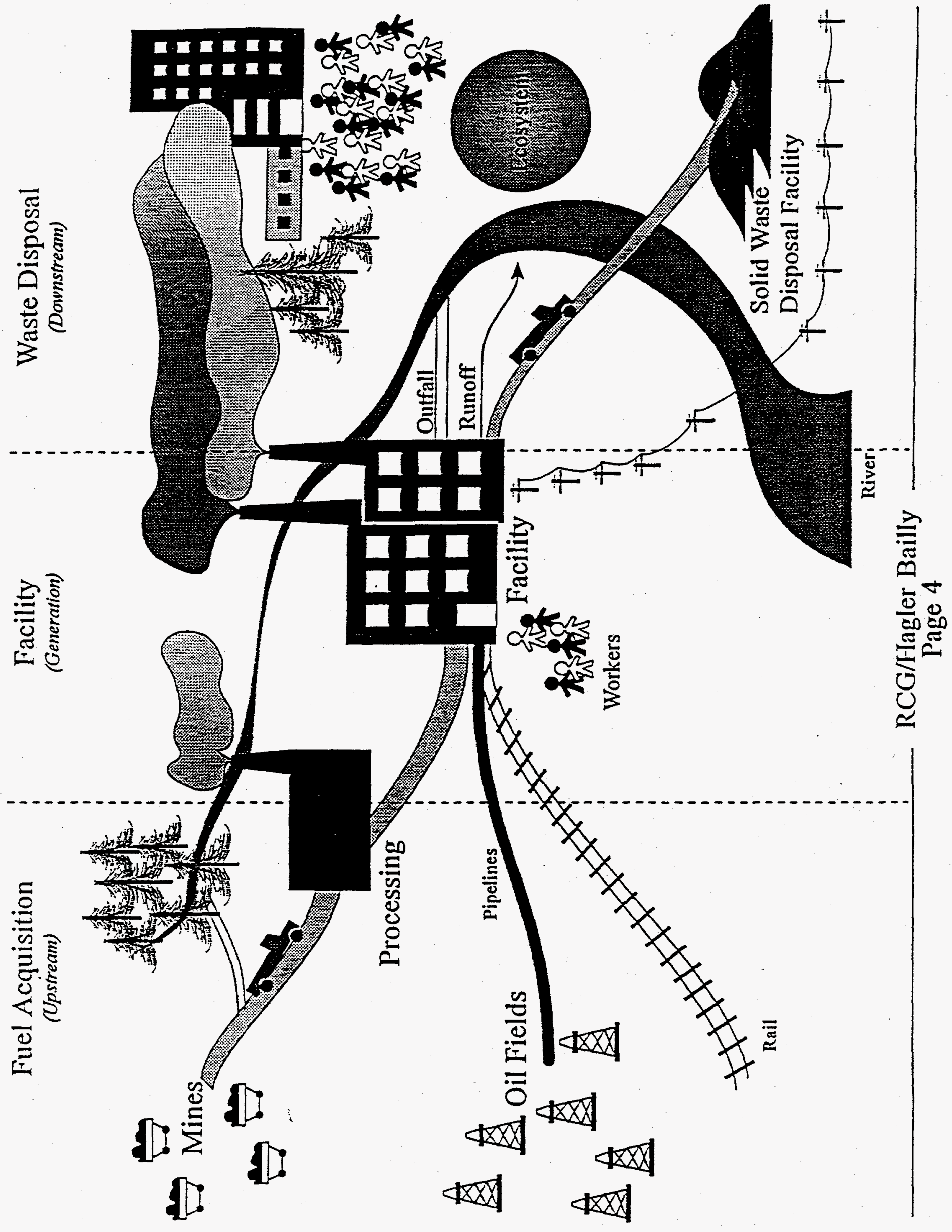




\section{FOUR MAJOR TASKS}

1. Externality Taxonomy and Screening

Report: December, 1993

2. Methodology

Report: November, 1994

3. EXMOD Model and Manuals

Report: December, 1994

4. Case Studies

Report: December, 1994

Page 5 


\section{EXTERNALITY TAXONOMY AND SCREENING}

- Six Major Groups

- Air, water, land, waste, radioactive, other

D Many Endpoint Models Within Each Group

Example:
Air
$\mathrm{NO}_{\mathrm{x}}$, Ozone, Lead, $\mathrm{PM}_{10}, \mathrm{SO}_{2}, \mathrm{SO}_{4}$, Mercury, Air Toxics, ...
$\mathrm{PM}_{10}$
Mortality, RHA, Asthma...
Visibility, Soiling...

- 160 Externality Groups and Over 300 Externality

Endpoints Screened into 4 Categories:

- Category 1: Develop damage function analysis for endpoint

- Category 2: Assigned $\$ 0$

- Category 3: Deferred (small values or limited literature plus high costs)

- Category 4: Insufficient literature or data 


\section{THE EXTERNALITY LIBRARY}

- Over 1,800 Documents

- Computerized Referencing Using "Endnote"

- Keyworded

- Search on author, keywords, journal

- Compatible with WordPerfect, Microsoft Word

RCG/Hagler Bailly

Page 7

A -68 


\section{Externality Screening Categories}

Source of Impacts

Externality Group

\section{Category I: Additional Quantification}

\section{Air}

Particulate matter (including secondary sulfate formed from $\mathrm{SO}_{2}$ emissions)

$\mathrm{NO}_{\mathrm{x}}$, Nitrates

Lead emissions

Mercury emissions

Mortality, Morbidity, Visibility,

Ambient ozone

Acid aerosols

Acid deposition

$\mathrm{SO}_{2} /$ Acid deposition

Air toxics emissions

Water

Entrainment/Impingement

Water consumption

Fisheries

Aquatic ecosystems

Groundwater impacts

Surface water -- chemical and metals discharges

Surface water -- thermal plume

Mortality/morbidity

Visibility

Mortality/morbidity

Morbidity

Mortality/morbidity

Commercial crops

Mortality/morbidity

Visibility

Aquatic impacts

Galvanized steel

Cancer mortality and morbidity

Nuclear

Normal operational radioactive

Cancer risks releases

Radioactive waste -- low level

\section{Cancer risks}




\section{Externality Screening Categories}

(Continued)

Source of Impacts

Externality Group

\section{Category I: Additional Quantification}

\begin{tabular}{|l|l}
\hline Radioactive waste -- high level (on- & Cancer risks \\
\hline
\end{tabular}

site storage)

Nuclear accidents

Loss of open space

services/terrestrial

Land

Facility land use -- all facilities including access roads and transmission lines

Solid waste disposal -- toxics in groundwater

Solid waste disposal -- land use and transportation on access roads

Aesthetics/noise/congestion

Other

Fuel extraction -- nuclear

Fuel processing -- nuclear

Transportation infrastructure

Energy security -- Oil

Electric transmission

Diminished resource value and health effects

Aesthetics, loss of open space services, terrestrial

Cancer risks

Mortality/morbidity

Road use -- coal plant

Energy security

Aesthetics

RCG/Hagler Bailly

Page 9 

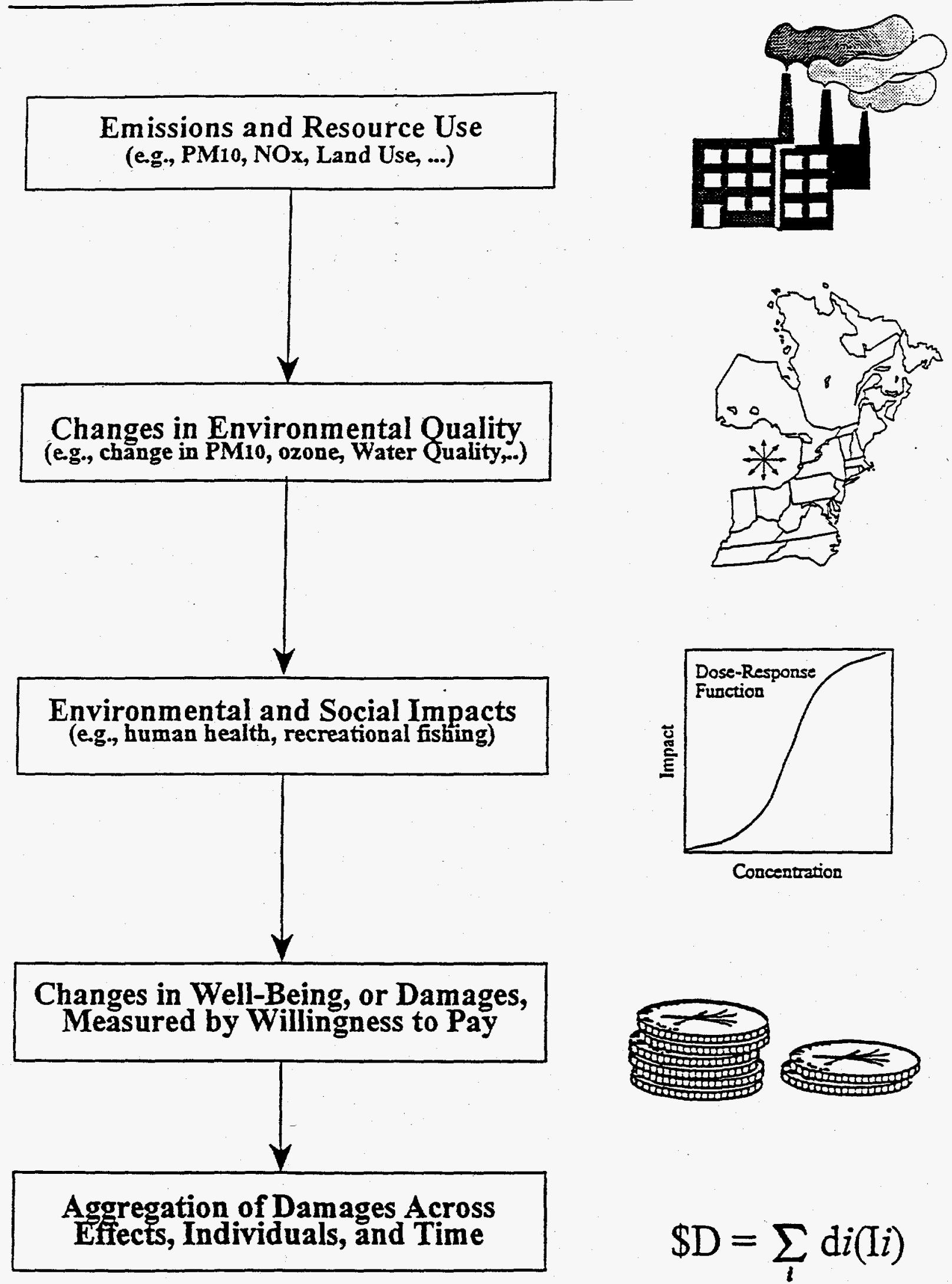

$\$ \mathrm{D}=\sum_{i} \mathrm{~d} i(\mathrm{I} i)$ 


\section{AIR DISPERSION MODELS}

- ISC2LT

Primary Particulates $<\mathbf{5 0 ~} \mathbf{~ k m}$

- SCREEN2

Peak Concentration

- SLIM3

Primary \& Secondary

Particulates $>\mathbf{5 0}$ km

- Ozone Limiting Method Short Range

- EKMA

Long Range 
Summary of Mortality and Morbidity Endpoints for $\mathbf{P M}_{10}$

\begin{tabular}{|l|l|}
\hline $\begin{array}{l}\text { Endpoint } \\
\text { under 65 }\end{array}$ & \multicolumn{1}{|c|}{ Primary Quantitative } \\
Sources
\end{tabular}

RCG/Hagler Bailly

Page 12 


\section{RECENT TIME-SERIES MORTALITY STUDIES}

\begin{tabular}{|c|c|c|c|}
\hline \multicolumn{4}{|c|}{ Recent Time-Series Mortality Studies } \\
\hline Study & $\begin{array}{l}\text { (1) } \\
\text { : }\end{array}$ & $\begin{array}{l}\text { Percent Increase } \\
\text { In Mortality for } \\
=\text { a } 10 \text { gg/m } \\
\text { Increase in } \\
\text { Daily } \text { PM }_{\mathbf{1}}^{2}\end{array}$ & S Study Location \\
\hline $\begin{array}{l}\text { Schwartz and } \\
\text { Dockery (ㅁ) }\end{array}$ & $\begin{array}{l}\text { Total Suspended } \\
\text { Particulates }\end{array}$ & $1.20 \% \pm 0.24$ & Philadelphia \\
\hline Schwartz (ㅇ) & $\begin{array}{l}\text { Total Suspended } \\
\text { Particulates }\end{array}$ & $0.99 \% \pm 0.26$ & Detroit \\
\hline $\begin{array}{l}\text { Schwartz and } \\
\text { Dockery }(\underline{7})\end{array}$ & $\begin{array}{l}\text { Total Suspended } \\
\text { Particulates }\end{array}$ & $0.64 \% \pm 0.20$ & Steubenville, $\mathrm{OH}$ \\
\hline Schwartz (9) & $\mathrm{PM}_{10}$ & $1.10 \% \pm 0.09$ & Birmingham, AL \\
\hline $\begin{array}{l}\text { Dockery et al. } \\
(\underline{10})\end{array}$ & $\mathrm{PM}_{10}$ & $1.51 \%=0.70$ & St. Louis \\
\hline Pope et al. (11) & $\mathrm{PM}_{10}$ & $1.54 \% \pm 0.37$ & Utah Valley, UT \\
\hline $\begin{array}{l}\text { Plagiannakos } \\
\text { and Parker }(12)\end{array}$ & Sulfate Aerosols & $0.98 \% \pm 0.49$ & $\begin{array}{l}\text { Southern } \\
\text { Ontario, Canada }\end{array}$ \\
\hline Fairley (13) & $\begin{array}{l}\text { Coefficient of } \\
\text { Haze }\end{array}$ & $1.02 \%=0.37$ & $\begin{array}{l}\text { Santa Clara } \\
\text { County, CA }\end{array}$ \\
\hline $\begin{array}{l}\text { Schwartz and } \\
\text { Marcus (14) }\end{array}$ & British Smoke & $0.31 \% \pm 0.02$ & London, England \\
\hline \multicolumn{4}{|c|}{$\begin{array}{ll}\text { Notes: } & 1.15) \text { Conversions used are as follows: } .55^{*} \mathrm{TSP}= \\
\mathrm{PM}_{10} & (\underline{1,15})\end{array}$} \\
\hline
\end{tabular}


Health:

Cost of illness $=$ medical cost + work loss CVM WTP $=\mathrm{COI}+$ inconvenience, pain, and suffering

Crops/Forests: Economic surplus based on mathematical programming models

Materials: Market prices, behavioral analysis, CVM WTP

Visibility:

CVM WTP, Hedonic property pricing

Recreation:

Travel cost models, CVM WTP

Land Use: Hedonic property pricing, land values 
- Each Endpoint Model Has:

- Effects (dose-response) computations

- Economic computations

- Uncertainty analyses

- Facility Characteristics and Endpoint Model Inputs

- Fixed assumptions

- User required inputs

- Default inputs (may be overridden) 
- Electricity Resource Characteristics

- Lifetime

- Average capacity factor

- Heat rate

- Miles of transmission line to grid

- Miles of access road

- Fuel transportation method

- Land use (acres $/ \mathrm{kWh})$

- Equipment Characteristics

- Control efficiencies

- Removal efficiency

- Cooling system

- Stack height, diameter

- Exhaust gas velocity, temperature 
- Fuel

- Source location

- Heat content

- Air Emissions (lbs/MMBtu)

- $\mathrm{SO}_{\mathrm{X}}, \mathrm{NO}_{\mathrm{X}}, \mathrm{CO}, \mathrm{TSP}, \mathrm{PM}_{10}$,

VOC, $\mathrm{CO}_{2}$, air toxics, lead, mercury

- Water Emissions and Water Use

- Discharge rate

- Discharge mixing zone

- Water body type (use/discharge)

- Consumption rate 


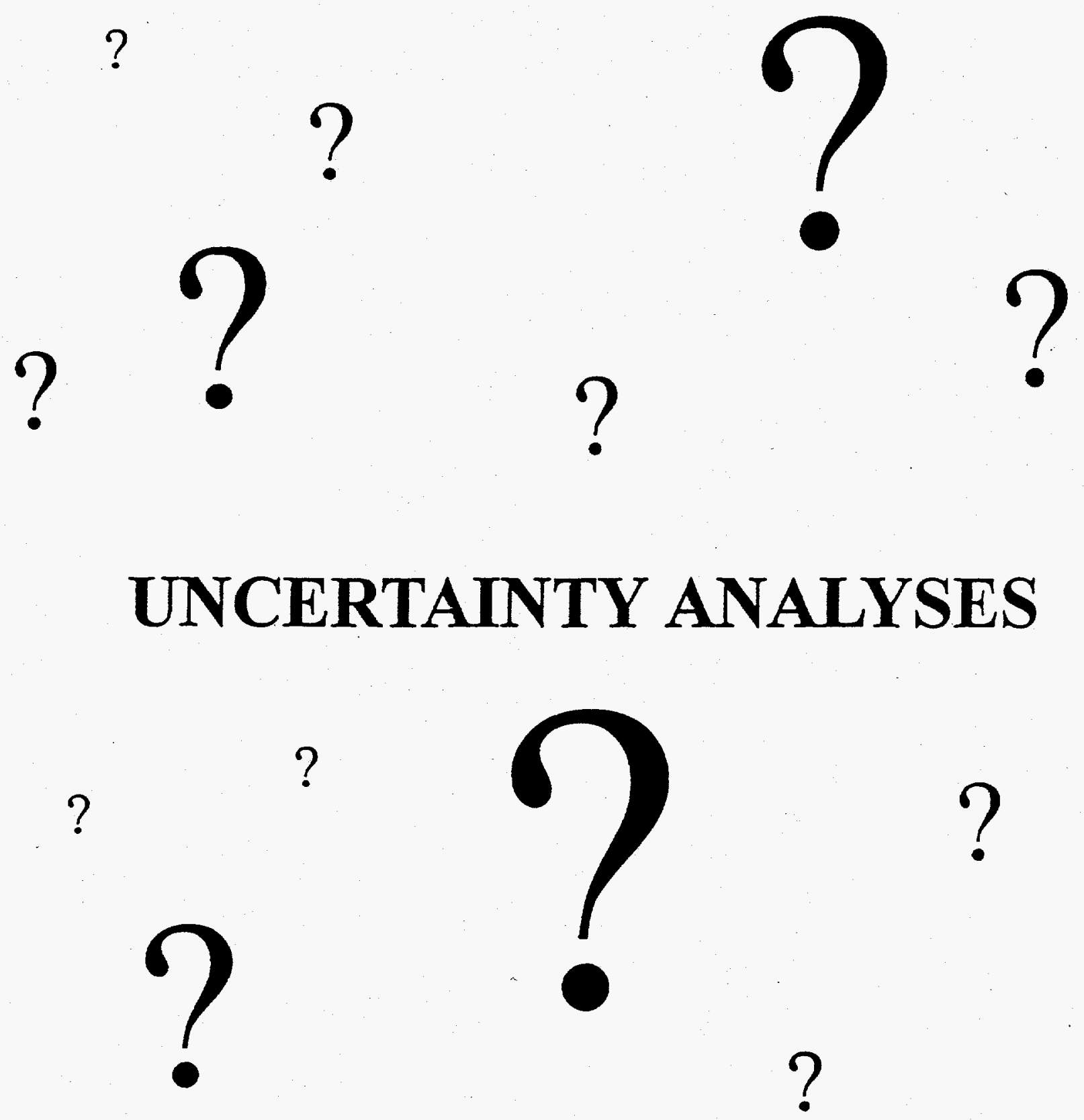

? 
- Summary Table of Omissions, Biases, and Uncertainties

- Key sources

- Known direction and magnitude of bias

- Overall impact

- Uncertainty Analysis

- Low, central, high values for selected variables and probabilities

- Error propagation algorithms

- Sensitivity Analysis

- User override of required inputs and many default assumptions

- User override of selected uncertainty parameters 


\section{EXTERNALITIES MODEL (EXMOD)}

\begin{tabular}{|c|c|c|c|}
\hline System & Data & Execution & Results \\
\hline $\begin{array}{l}\text { Help F1 } \\
\text { About EXMOD } \\
\text { Model Setup } \\
\text { System Info } \\
\text { File Management }\end{array}$ & $\begin{array}{l}\text { Sites } \\
\text { Receptor Cells } \\
\text { Facilities } \\
\text { Endpoint Group } \\
\text { Impact Valuation } \\
\text { Non-Quantified }\end{array}$ & $\begin{array}{l}\text { Run Model } \\
\text { Data Input Report } \\
\text { Intermediate Results }\end{array}$ & $\begin{array}{l}\text { Geographic Summary } \\
\text { Source Summary } \\
\text { Source Detail } \\
\text { Impact Detail } \\
\text { Input Data Summary } \\
\text { Dollar per Ton }\end{array}$ \\
\hline & $\begin{array}{l}\text { Bibliography } \\
\text { Taxonomies }\end{array}$ & & \\
\hline
\end{tabular}

Requires 386 PC with 4K RAM and $40 \mathrm{MB}$ hard drive 


\section{TYPICAL INPUT SCREEN}

\section{Endpoint Model Data Window}

EndPoint Model Group

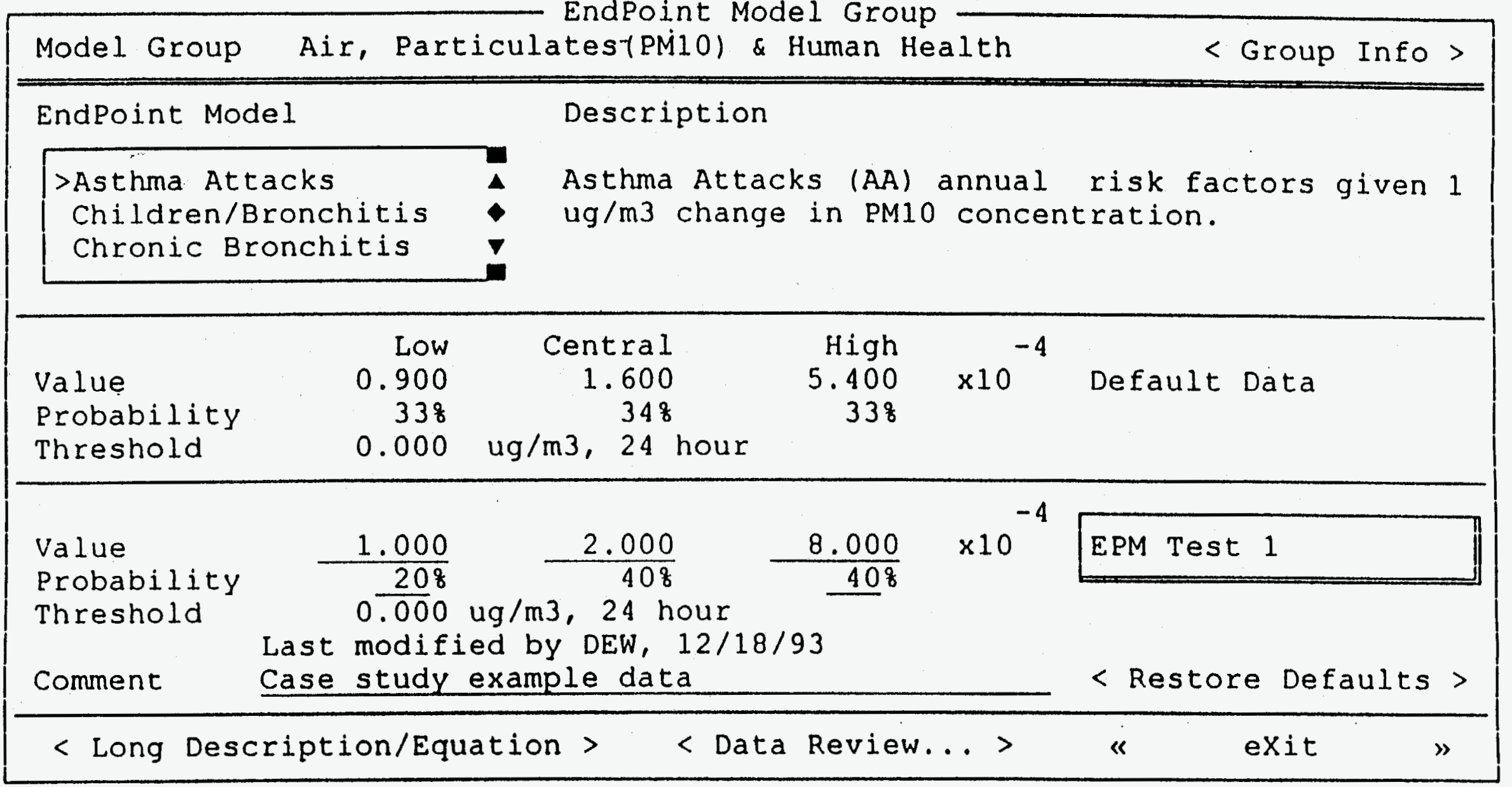


- Physical Impacts

- Selected impacts, annual average

- Dollar Damages

- Mills $/ \mathrm{kWh}$

- Total damage

- $\$$ Ton

- Time Periods

- Present value

- Annual average

- Geographic Areas

- Local

- Rest of region

- Rest of state

- Out of state
(In-state $\leq 30 \mathrm{~km}$ )

(In-state $30-80 \mathrm{~km}$ )

- Aggregation of Damages

- Individual externalities

- Major groups (air, water, land/waste, other)

- Total 


\section{EXMOD OUTPUT}

\section{New York Environmental Externalities Model DAMAGES SOURCE SURMARY \\ (Annual Average Mills/kWh)}

Site: JFK Airport

Facility: Oil Distillate Combined Cycle (OCC) Scenario: User Baseline Run Date: 09/19/94 Time: 10:21:18 User: CML

Capacity: $\quad 210 \mathrm{MW}$ Lifetime: $\quad 30$ yrs Annual Prod: $551 \mathrm{GWh}$

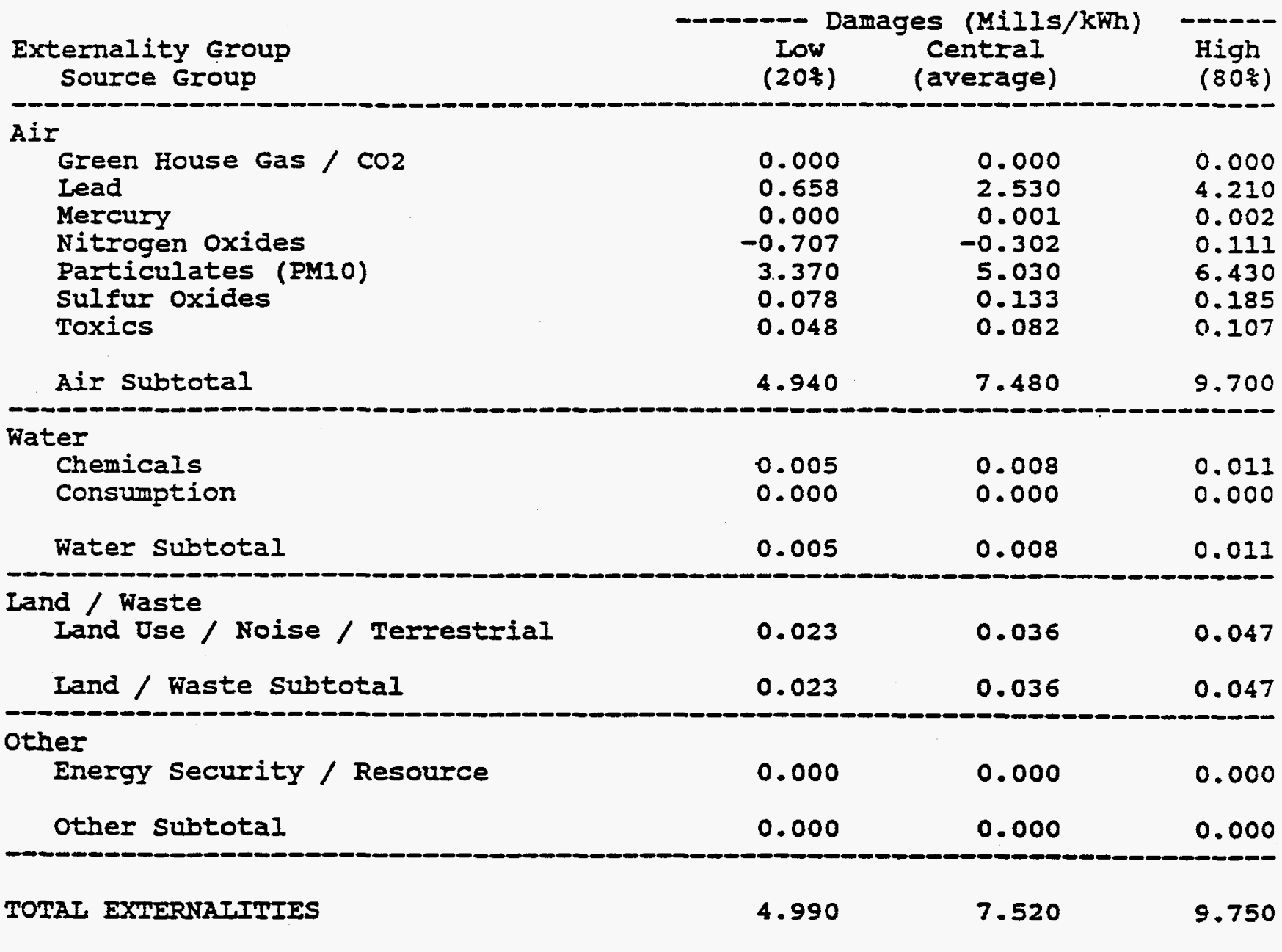

Notes: Iow and high totals may not sum because of Central Iimit Theorem. $1 \mathrm{mill}=\$ 0.001$

Source: EXHOD V0.9e by Tellus Institute and RCG/Hagler Bailly 
- Air Models

- Hourly particulate and ozone models

- More ozone models

- Future baseline air quality

- Impacts and Valuation

- Effects thresholds

- Evaluation of large air quality changes

- $\mathrm{NO}_{\mathrm{x}}$ and other pollutant offsets, refinement of $\mathrm{SO}_{2}$ trading

- Add more endpoints, especially upstream impacts

- Greenhouse gas valuation

- Modeling

- Detailed grid of all locations in the model

- Expand geographic coverage

- Refined emissions characteristics; esp. air toxics

- Add more internal documentation 
A -86 


\section{ESTABLISHING APPROPRIATE BOUNDARIES AND PARAMETERS FOR TOTAL FUEL CYCLE ASSESSMENT}

Opening Presentation on Electric Vehicle Total Energy Cycle Analysis Presented by Jim Ohi, National Renewable Energy Laboratory

Followed by a Boundaries and Parameters Working Group Facilitated by Kenneth W. Boras, BCS, Incorporated; Michael Samsa, Gas Research Institute 
A -88 


\title{
Electric Vehicle Total Energy Cycle Assessment
}

\author{
Jim Ohi \\ Total Fuel Cycle Analysis Workshop \\ Austin, Texas
}

October 6, 1994

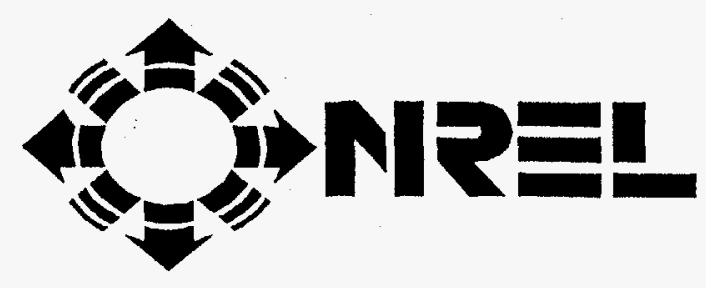




\section{Outline of Presentation}

\section{Background}

TECA

EVTECA

Issues

Methodology

Analysis

Policy 


\section{Total Energy Cycle Analysis (TECA)}

- Objectives

- To develop and apply TECA to identify environmental tradeoffs associated with conventional and renewable energy technologies

- To provide honest evaluation of EE/RE technologies and competing energy technologies 


\section{TECA Examines Environmental Effects From a "Complete System" Perspective}

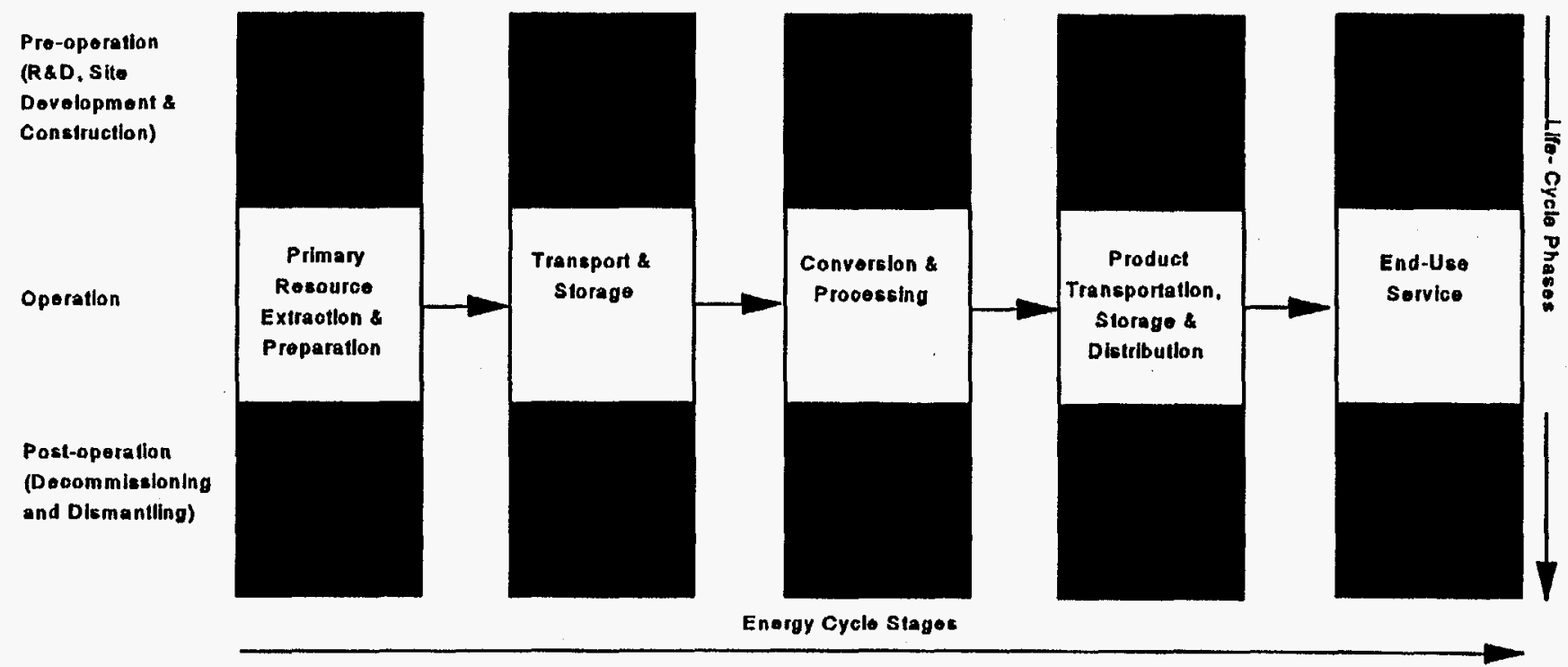




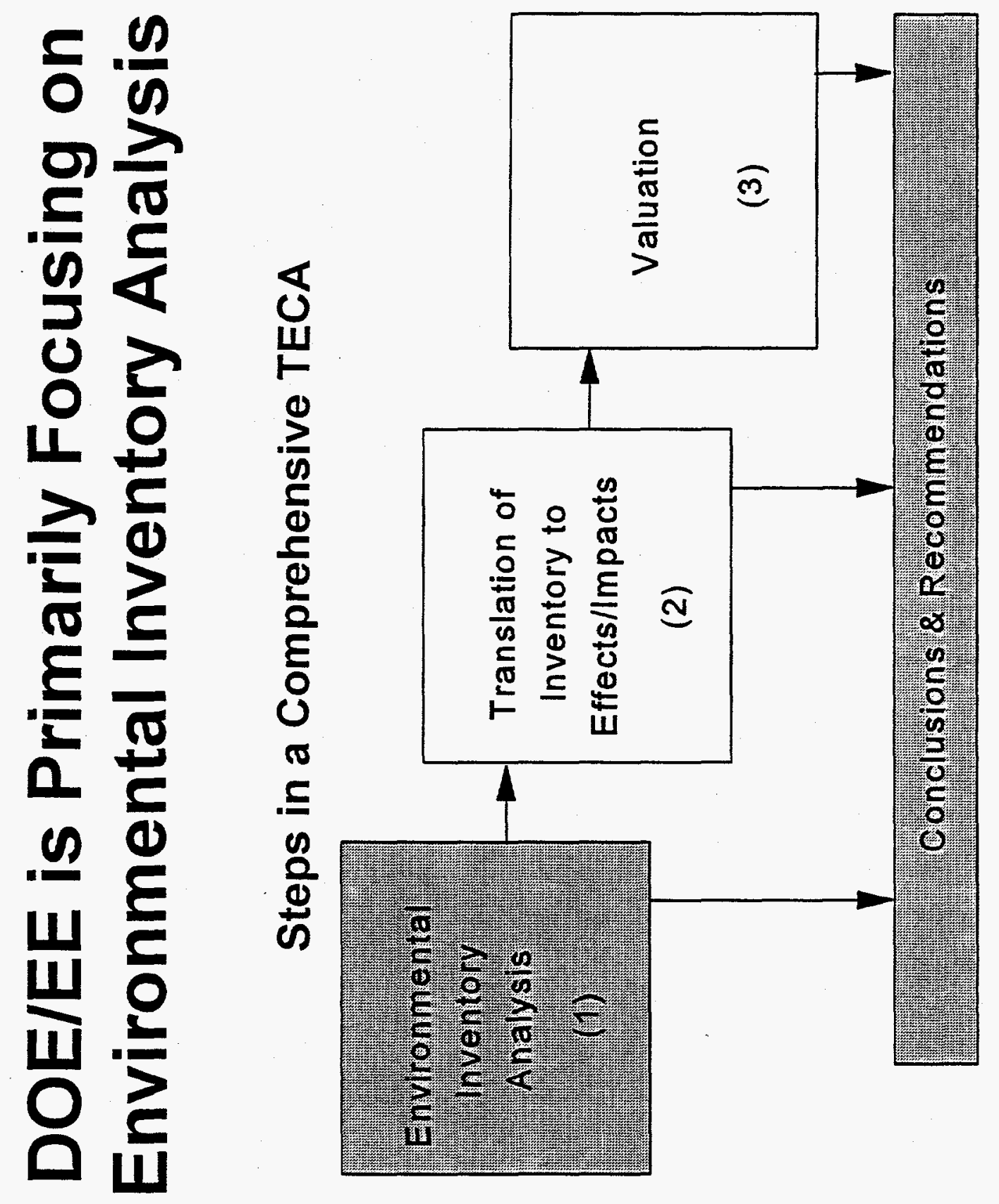




\section{Electric Vehicle Total Energy Cycle Analysis (EVTECA)}

\section{Objectives}

Quantify emissions and energy-use associated with EVTEC for several scenarios of EV use and compare to those of conventional vehicle benchmark,

Refine methodology and benchmark assessments developed for biomass-to- ethanol TECA, and

Test applicability and relevance of TECA by integrating the EVTECA directly to actual air quality management process 


\section{Management}

Funded and Managed by DOE

Assistant Secretary for Energy Efficiency and Renewable Energy

Office of Planning and Assessment

Office of Transportation Technologies

Office of Utility Technologies

Assistant Secretary for Nuclear Energy

Multi-laboratory Effort involving NREL, ANL, PNL

One-year (+) Project 


\section{Tasks}

Battery and Vehicle Characterization-ANL Benchmark Refinement-ANL

Data Analysis and Modeling-PNL

Scenario Development-NREL

Utility Analysis-NREL

Peer Review Administration-NREL

Air Quality Modeling/Management Coordination-NREL

Presentation of Results-PNL 


\section{Peer Review Administration}

Integrate Peer Review into Project Management

Use EV Battery Readiness Working Grouas Basis for National Peer

Review Board

battery developers, automobile manufacturers, chemical processing companies, DOE, DOT, EPA, EPRI

Technical peer review by specialists (utility analysis, EV characterization, RFG characterization, etc.)

Local and regional entities (e.g., CEC, CARB, MVMA, SCE, etc., for LA region) for regional peer review 


\section{Air Quality Modeling/Management Coordination}

Coordinate all EVTECA tasks as needed with the SCAQMD

Exchange information and data

Develop scenarios with the SCAQMD

SCAQMD performs air quality modeling

SCAQMD uses EVTEC data for EIR required by CEQA 


\section{Scenario Development}

Selection criteria

utility fuel-mix and dispatch characteristics

transportation patterns and utility characteristics that influence EV charging patterns

potential of extrapolating regional scenarios to estimate nationwide impacts

data availability and interest of regional agencies/organizations in cooperation with the EVTECA team. 


\section{Scenario Development}

Select Four Regions

Los Angeles [South Coast Air Quality Management District (SCAQMD)]

Other Candidate Areas

Washington, DC/Baltimore (on-going TECA project, utility/local govt. interest)

Detroit (collaboration with Detroit Edison in the nation's automobile capital)

Seattle (hydroelectric power)

New York City (NYSERDA 3-year study of the externalities of power generation)

Phoenix (warm area, active utility EV programs )

Chicago (mid-west, nuclear generation component)

Denver (high-altitude, winter $\mathrm{CO}$ problem with little public transit )

Houston (south(west), warm area, natural gas/oil) 
EVTECA

Scenario Development

City

Pop.

$\mathrm{Oz}$

$\mathrm{CO}$

ZEV

$\Sigma$

Atlanta

3

Baltimore

$\begin{array}{ll}2 & 1 \\ 3 & 2 \\ 2 & 1 \\ 2 & 1 \\ 2 & 2 \\ 4 & 1 \\ 2 & 1 \\ 2 & 2 \\ 1 & 4 \\ 1 & -1 \\ 2 & 1 \\ 4 & 1 \\ 5 & 5 \\ 4 & 1 \\ 4 & 3 \\ 3 & 2 \\ 2 & 1 \\ 2 & 2 \\ 2 & 1 \\ 3 & 2 \\ 3 & 1\end{array}$

1

2

Baton-Rouge

3

Beaumont-Port Art.

Boston

Chicago

"Greater CN"

D. C.

Denver

Detroit

El Paso

Houston

L. A

Milwaukee

N. Y.

Philadelphia

Providence

Sacramento

San Joaquin Valley

San Diego

Ventura

\begin{tabular}{|c|c|c|}
\hline Rating & Ozone & ZEV \\
\hline 5 & Extreme & Enacted \\
\hline 4 & Severe (1) .19 to $.28 \mathrm{ppm}$ & Enacted / Chal. \\
\hline 3 & Severe (2) .18 to $.19 \mathrm{ppm}$ & Regional Iniative \\
\hline 2 & Serious (1) & $\mathrm{red}$ \\
\hline
\end{tabular}


EVTECA

Scenario Development

\begin{tabular}{|c|c|c|c|c|c|c|c|c|c|c|c|c|}
\hline & & & Fuel $\mathrm{M}$ & Mix Di & ersity & & Clim & ate Rating & & & & \\
\hline & City & $\begin{array}{l}\text { Regional } \\
\text { Diversity }\end{array}$ & Coal & Nuc & Ren & $\begin{array}{l}\text { Nat. } \\
\text { Gas }\end{array}$ & $\begin{array}{l}\text { Heating } \\
\text { oDays }\end{array}$ & $\begin{array}{l}\text { Cooling } \\
\text { oDays }\end{array}$ & $\Sigma$ & $\begin{array}{c}\text { Energy } \\
\text { Cap.' }\end{array}$ & $\begin{array}{l}\text { Annual } \\
\text { Peak }^{2}\end{array}$ & $\begin{array}{l}\text { Daily } \\
\text { Peak }\end{array}$ \\
\hline & L. A. & $\mathrm{CA}$ & C & $\mathrm{N}-$ & $\mathrm{R}+$ & & 1,458 & 727 & 2,185 & B & $S$ & $\mathrm{AM}$ \\
\hline & N. Y. & NE & C. & N- & R- & & 4,805 & 1,096 & 5,901 & $\mathbf{S}$ & w & PM \\
\hline & Chicago & MW & $\mathrm{C}+$ & $\mathrm{N}++$ & 0 & & 6,536 & 752 & 7,288 & $\mathbf{S}$ & W & PM \\
\hline & Boston & NE & C & $\mathrm{N}$ & R- & & 5,641 & 678 & 6,409 & $S$ & W/S & PM \\
\hline . & Baltimore & MA & $\mathrm{C}+$ & N- & 0 & & 4,707 & 1,137 & 5,844 & $S$ & $S$ & $\mathrm{AM}$ \\
\hline & Philidephia & MA & $\mathrm{C}+$ & $\mathrm{N}$ & 0 & & 4,954 & 1,101 & 6,055 & $S$ & w & PM \\
\hline & D. C. & MA & $\mathrm{C}+$ & 0 & 0 & & 4,047 & 1,549 & 5,596 & $S$ & W/S & $\mathrm{AM} / \mathrm{PM}$ \\
\hline & San Diego & $\mathrm{CA}$ & C & N- & $\mathrm{R}+$ & & 1,256 & 984 & 2,240 & B & $S$ & $\mathrm{AM}$ \\
\hline & Ventura & $\mathrm{CA}$ & $C$ & $N-$ & $\mathrm{R}+$ & & 2,300 & 1,100 & 3,400 & B & $S$ & $\mathrm{AM}$ \\
\hline & Sacramento & $\mathrm{CA}$ & C & N- & $\mathrm{R}+$ & & 2,749 & 1,237 & 3,986 & B & $S$ & $\mathrm{AM}$ \\
\hline & Detroit & MW & $\mathrm{C}+$ & 0 & R- & & & & & $S$ & & \\
\hline & Houston & SW & $\mathrm{C}+$ & N- & 0 & $\mathrm{G}++$ & 1,599 & 2,700 & & $S$ & $S$ & $\mathrm{AM}$ \\
\hline & Atlanta & So & $\mathrm{C}+$ & $\mathrm{N}$ & 0 & G- & 2,991 & 1,667 & & $S$ & $S$ & $\mathrm{AM}$ \\
\hline Scenario & ${ }^{\circ}$ mean $=4,890$ & ${ }^{1} \mathrm{~B}=\mathrm{B}$ & lance & $\mathrm{S}=\mathrm{St}$ & Irplus & & ${ }^{2} S=$ & Summer & $\mathrm{W}=\mathrm{Win}$ & & & \\
\hline 1st choice: & $\begin{array}{l}\text { L. A. } \\
\text { Chicago } \\
\text { D. C. } \\
\text { Houston }\end{array}$ & $\begin{array}{l}\text { R+; Balance; } \\
\text { N+I; Surplus; } \\
\text { C+; Surplus; } \\
\text { G++; Surplus; }\end{array}$ & $\begin{array}{l}\text { extreme } \\
\text { extrem } \\
\text { noderat } \\
\text { extrem }\end{array}$ & $\begin{array}{l}\text { ely mo } \\
\text { nely co } \\
\text { te clim } \\
\text { nely ho }\end{array}$ & $\begin{array}{l}\text { derate } \\
\text { Id rel } \\
\text { ate; } N \\
t ; S W\end{array}$ & $\begin{array}{l}\text { relati } \\
\text { ative to } \\
\text { iA loc } \\
\text { Ilocati }\end{array}$ & $\begin{array}{l}\text { ve to mear } \\
\text { mean; } M \\
\text { ation } \\
\text { ion }\end{array}$ & $\begin{array}{l}\text {; CA loca } \\
\text { W locatio }\end{array}$ & & & & \\
\hline 2nd choice: & $\begin{array}{l}\text { Atlanta } \\
\text { N. Y. } \\
\text { L. A. } \\
\text { Houston }\end{array}$ & & $3 \mathrm{rd} \mathrm{ch}$ & oice: & $\begin{array}{l}\text { Bo } \\
\text { L. } \\
\text { Atl }\end{array}$ & $\begin{array}{l}\text { ston } \\
\text { A. } \\
\text { anta }\end{array}$ & & & & & & \\
\hline
\end{tabular}




\section{Low EV Market Penetration Scenario Percent Sales New Passenger Vehicles and LD Trucks}

Year Los Angeles Chicago

Houston

WDC

1998

1999

2000

2001

2002

2003

2

2

5

5

5

10

2005

2006

2007

2008

2009

2010

10

10

10

10

10

10

0
0
0
1
1
2
2
3
3
3
3
3

0

0

0

0

1

1

2

3

3

3

3 


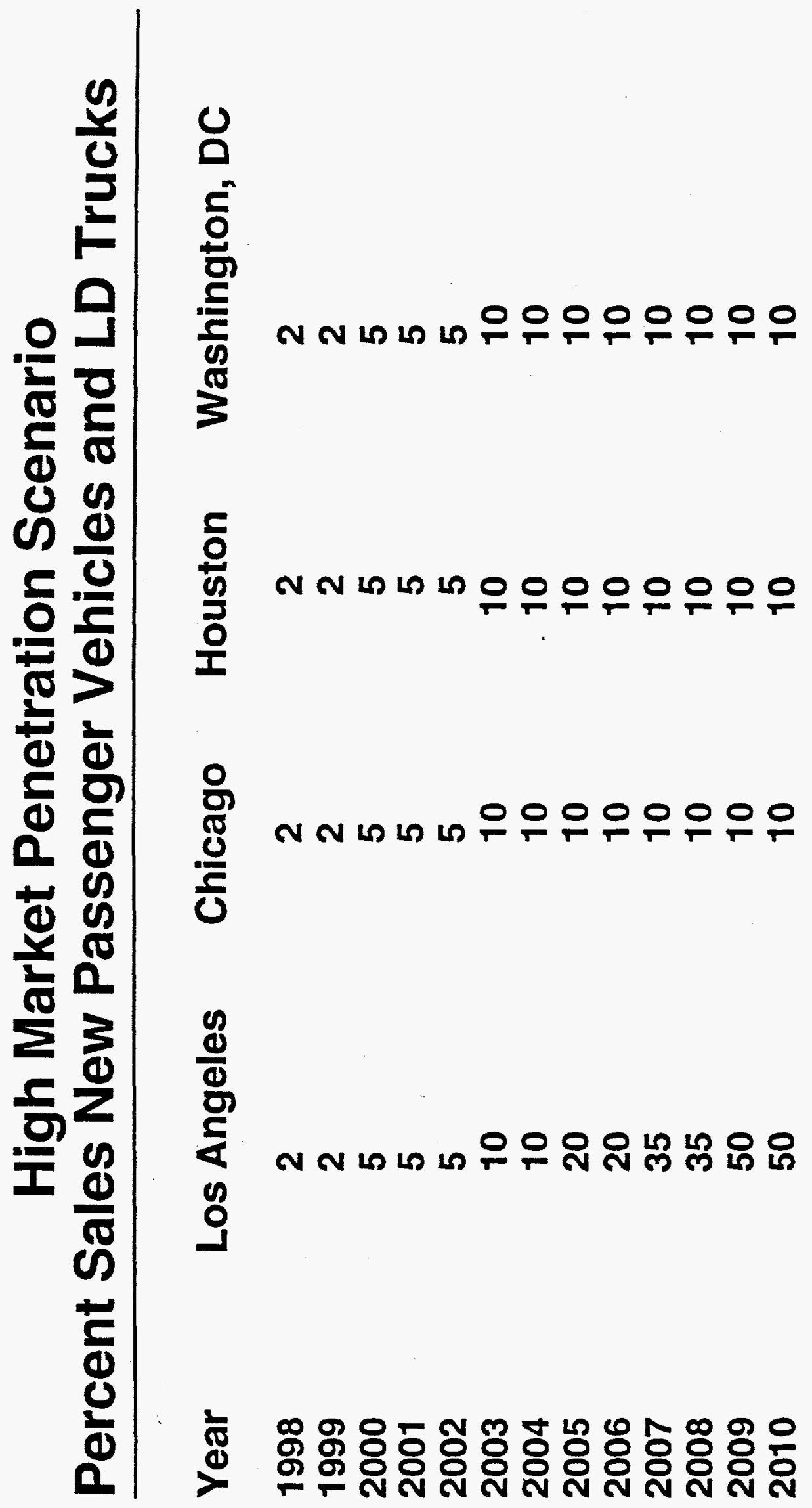




\section{EVTECA Utility Analysis}

- Four major tasks required

- Obtain utility data and validate

- Synthesize cumulative electric vehicle temporal power demand

- Perform dispatch calculations

- Developed emission-constrained dispatch to simulate the effect of 1990 CAAA

- Perform emission calculations 


\section{Project status}

- Base case scenarios (no EVs) completed

- Chicago and Houston EV scenarios completed

- Currently working on Los Angeles EV scenarios

- Los Angeles scenario forms basis for IRP deliverable

- Future agenda

- Complete the "unconstrained" EV scenarios (LA, D.C.)

- Run policy scenarios 


\section{TECAs: Where Are We Going?}

- EVTECA very complex

- multi-sectoral

- multi-regional

- multiple vehicle technologies

- utility dispatch/capacity modeling

- air quality/SIP issues 


\section{TECAs: Where Are We Going?}

- EVTECA tests analytic resources, capabilities

- methodology

- data and computational resources

- interagency/Interlaboratory coordination

- national/regional coordination 


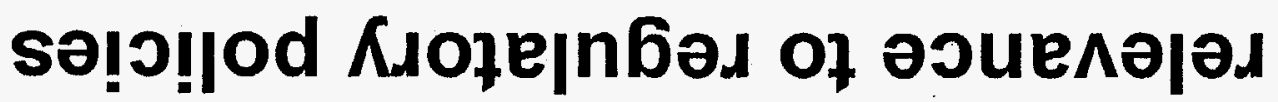

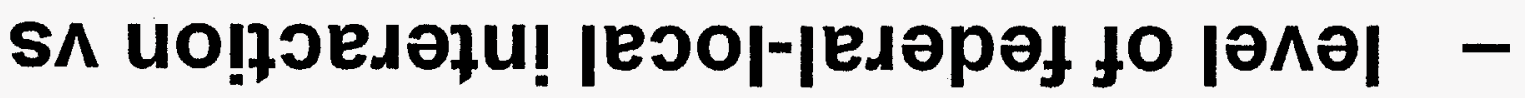

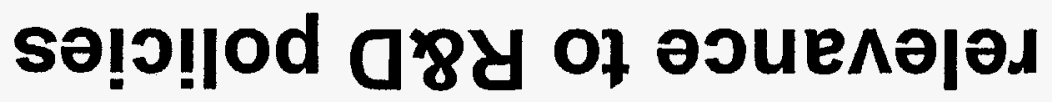
s^ Kł!suәfu! uo!feqndwos pue efep bu!puezs.sopun

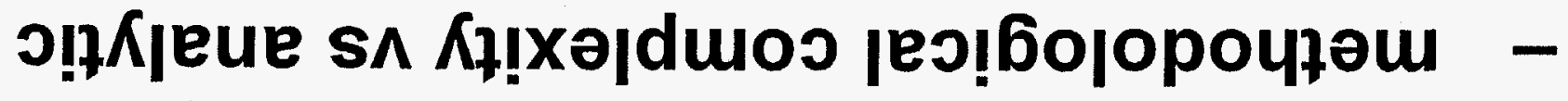
u! suanłod bu!̣s!u!u!p fo łu!̣od ssoss

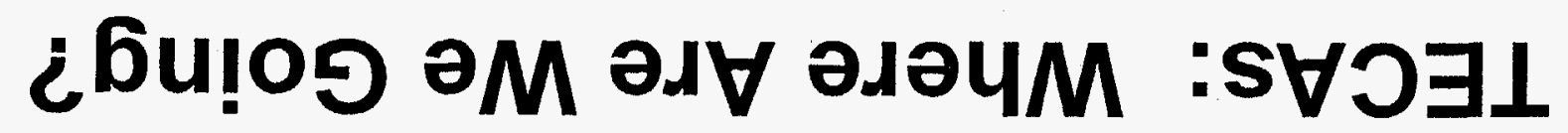




\section{TECAs: Where Are We Going?}

- Develop recommendations to DOE on whether to:

- address impact and valuation components of TECAs

- link environmental and energy policy through TECAs

- link to EH\&S risk minimization via EPA 


\section{Specify Limits of Methodological/Computation Complexity}

- analytic understanding

- relevance to R\&D policy

- relevance to environmental and energy regulatory policies 
A - 112 


\section{IDENTIFYING APPROPRIATE MODELS, METHODS AND APPROACHES FOR IMPLEMENTING TOTAL FUEL CYCLE ASSESSMENT}

Opening Presentation on Electric and Gas Cooling, Presented by Jim Block, Xenersys

Followed by a Models, Methods and Approaches Working Group Facilitated by Jay Zarnikau, Planergy and Mark Krebs, Southern Union Energy International 
A - 114 


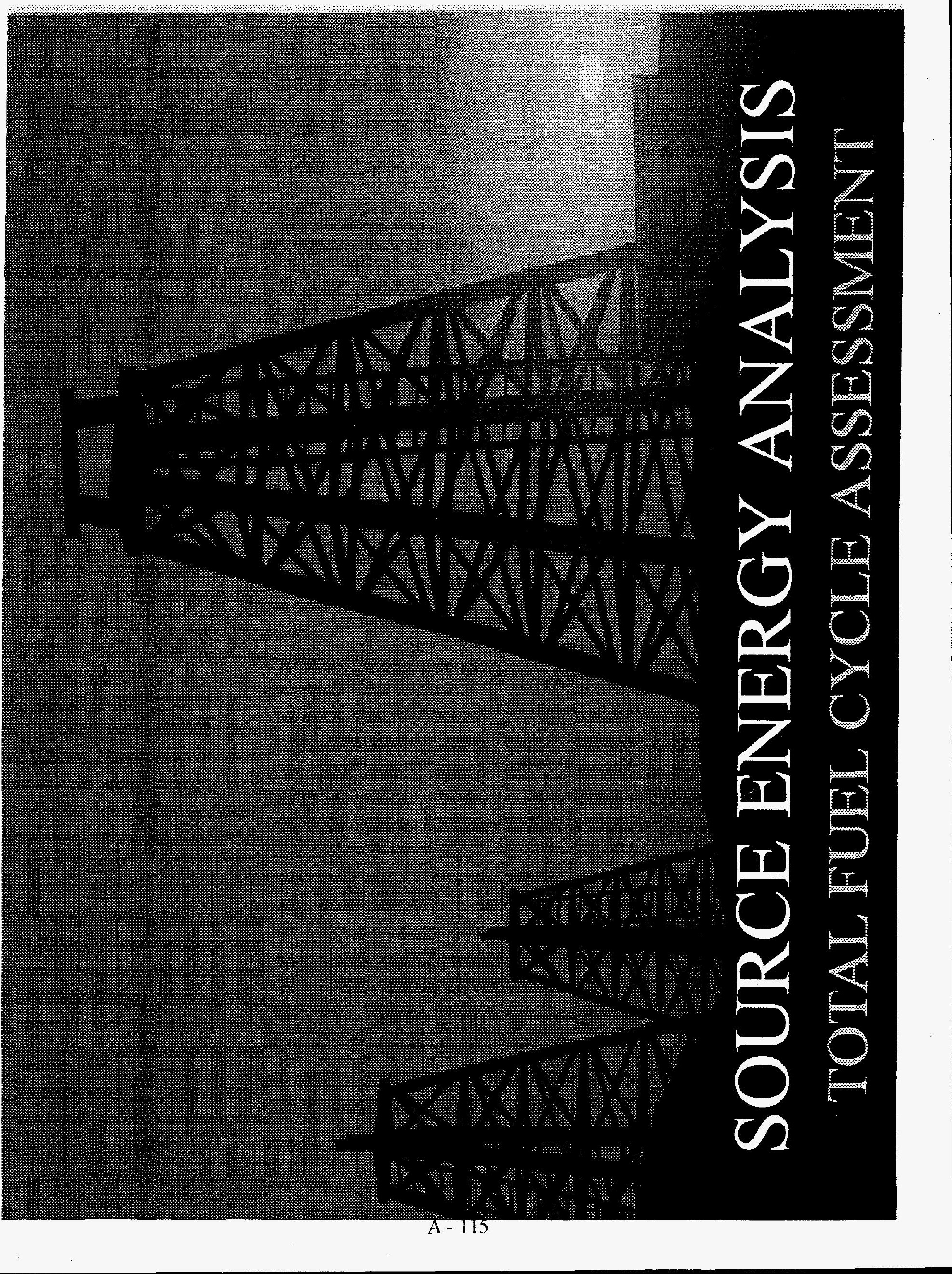




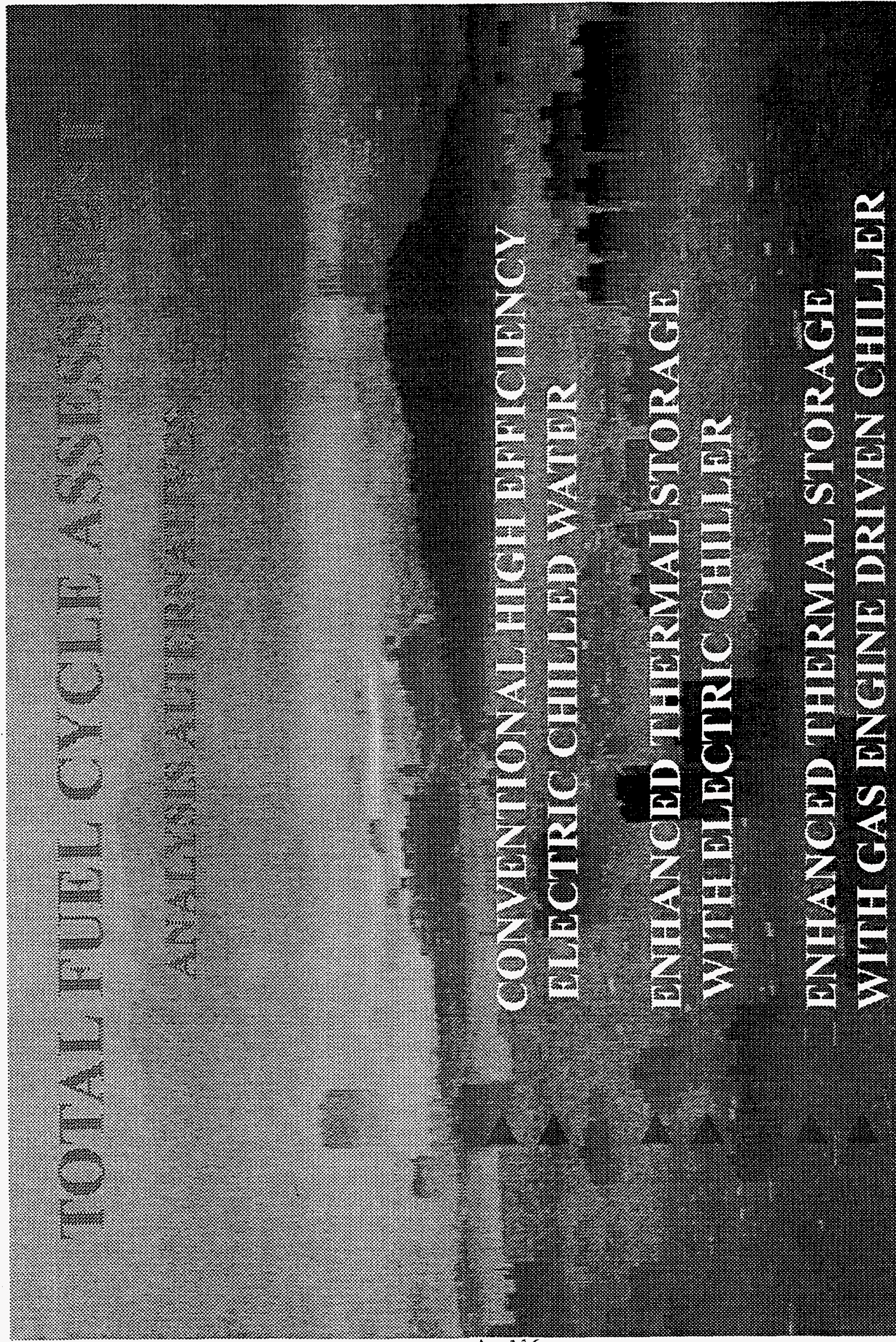




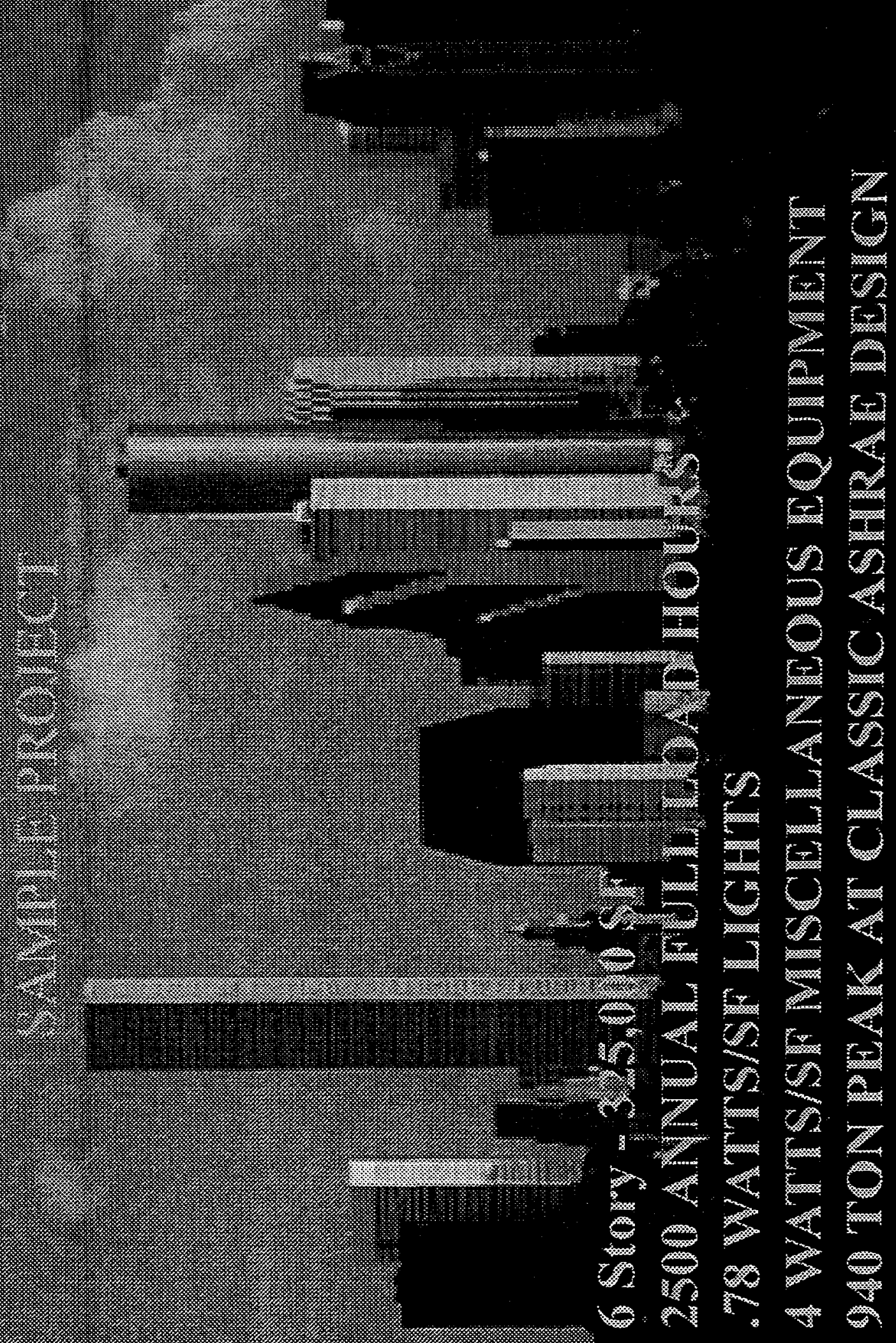




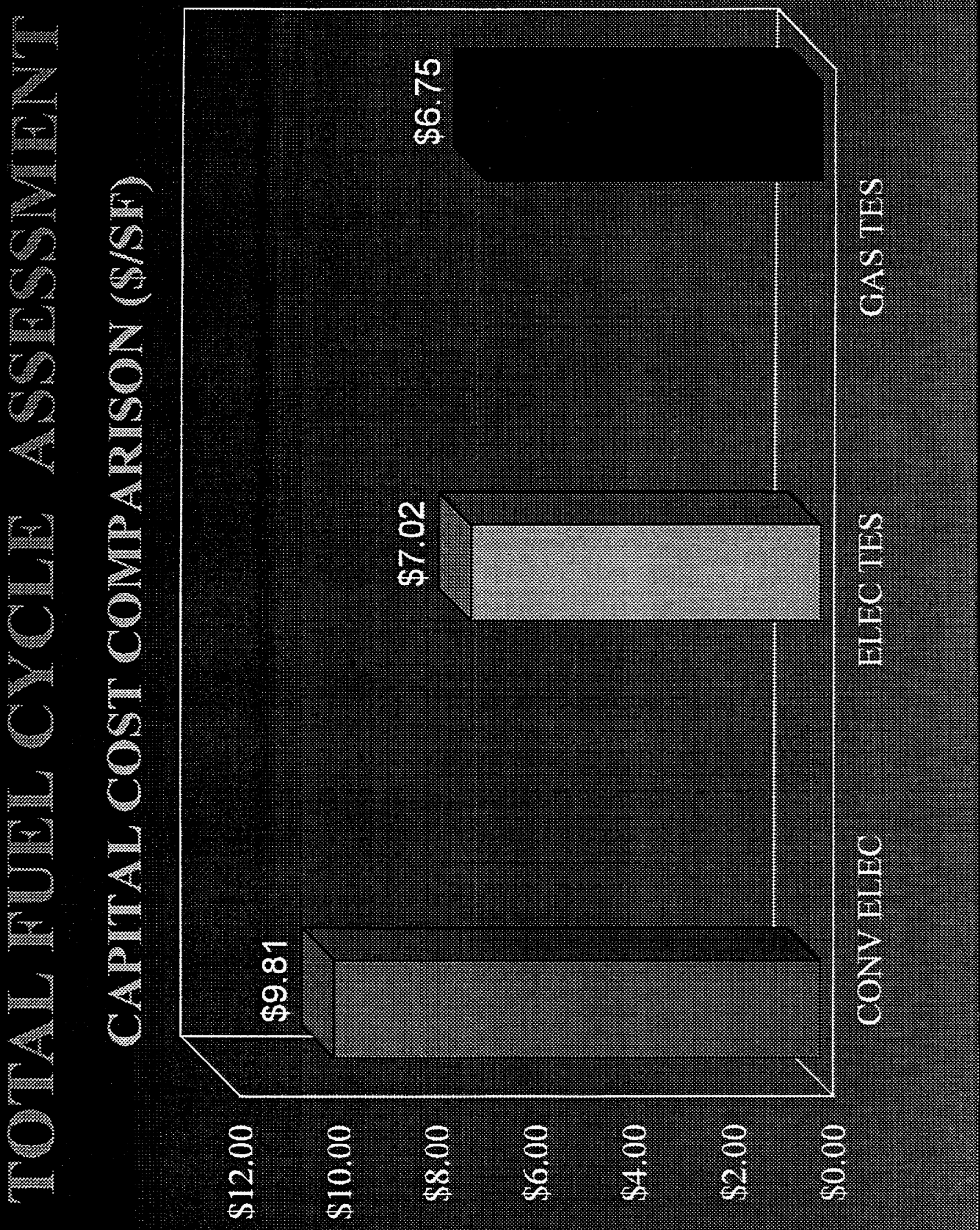




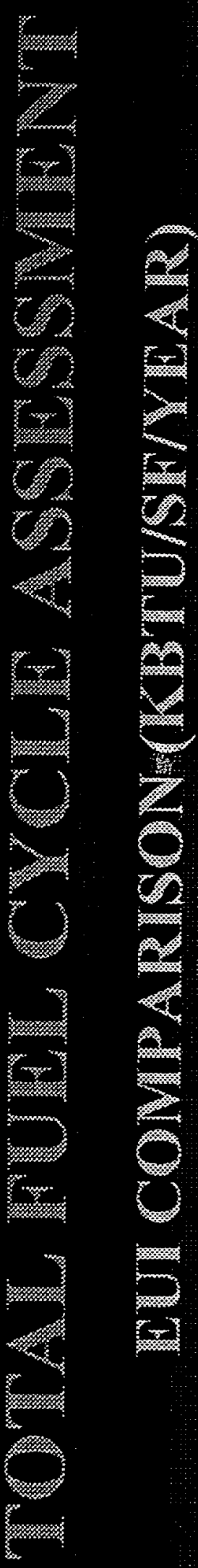

$\frac{9}{6}$

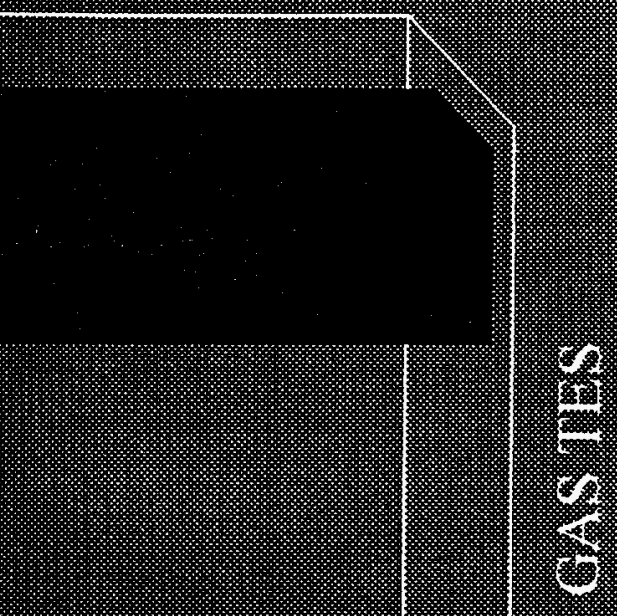




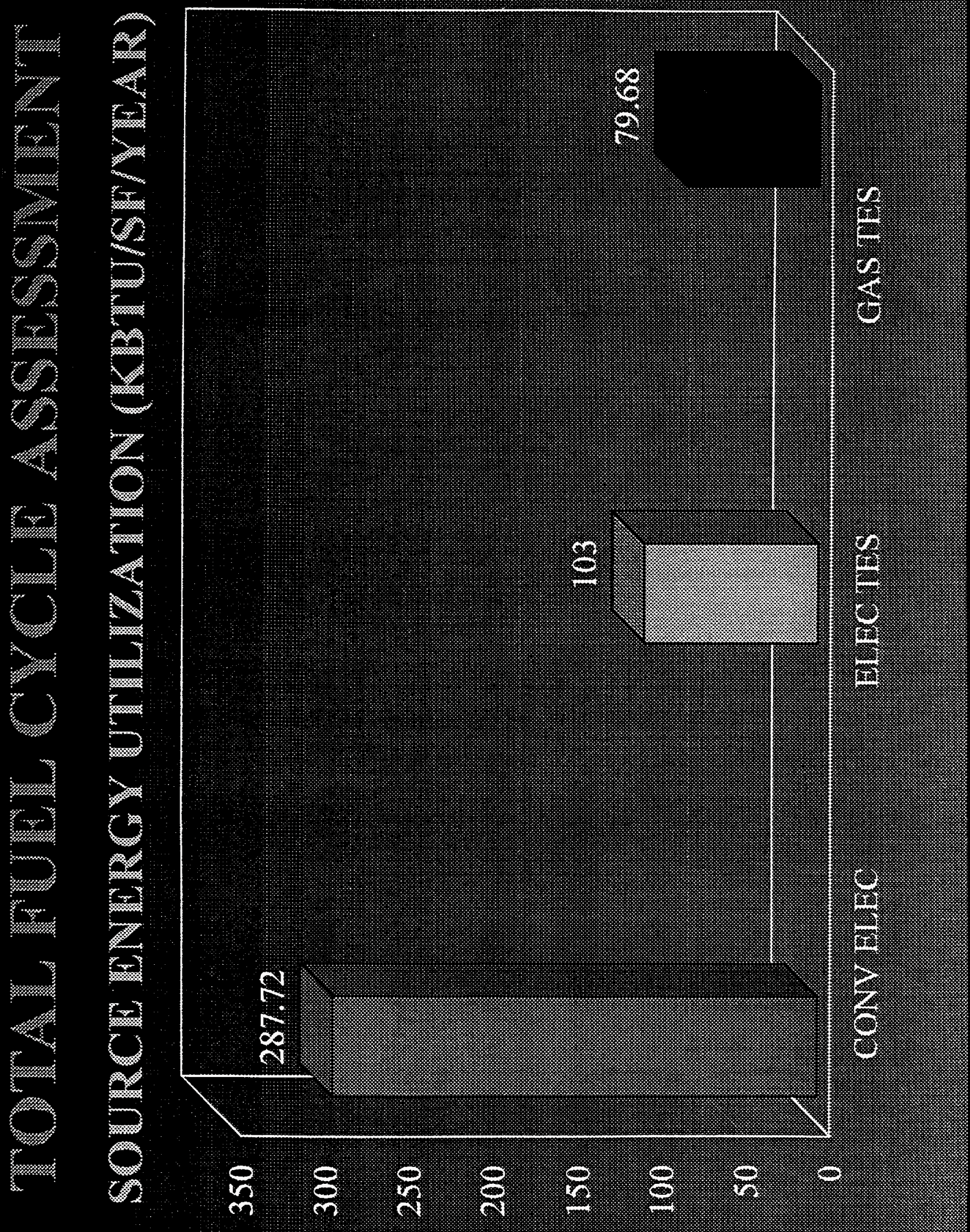



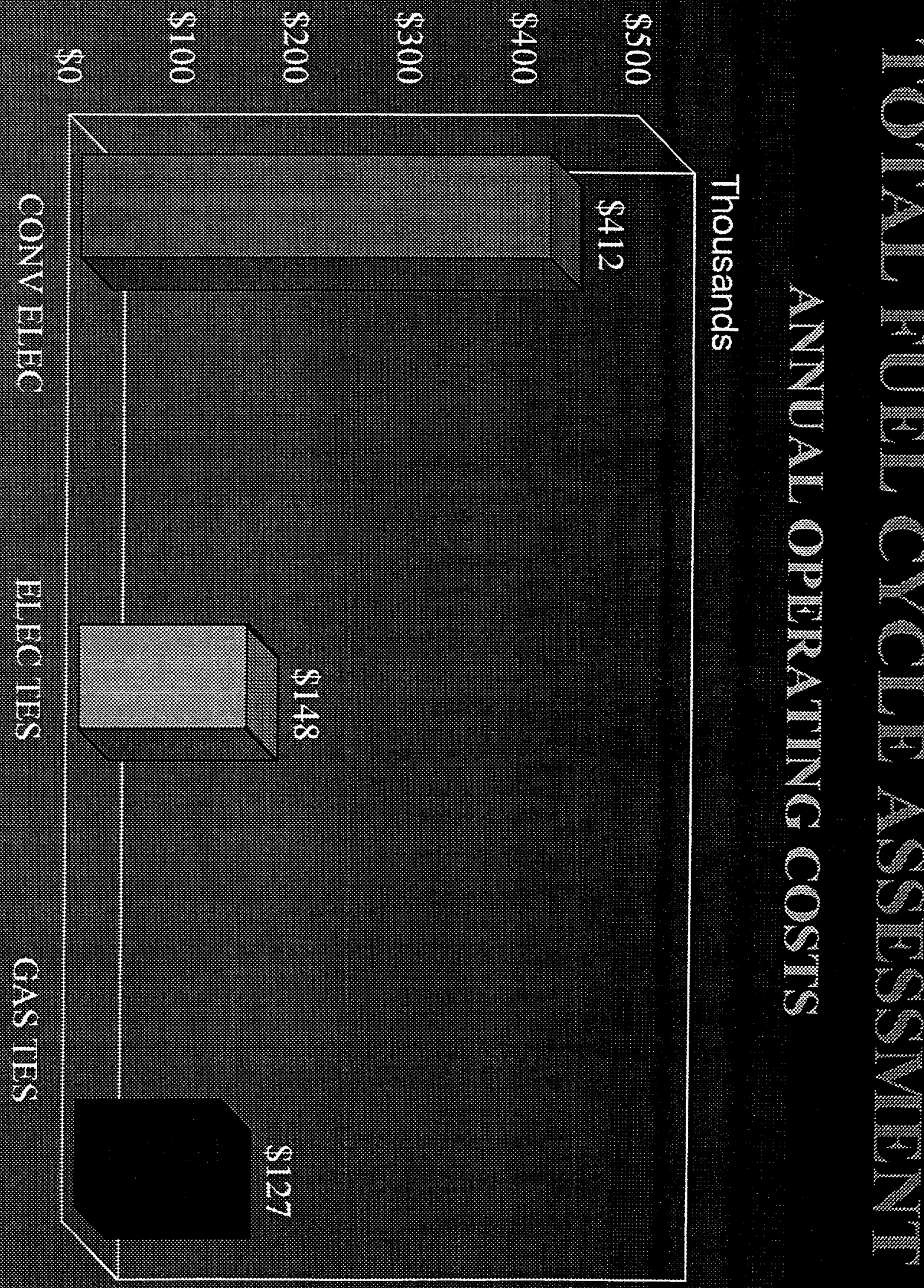

(m)

(3)

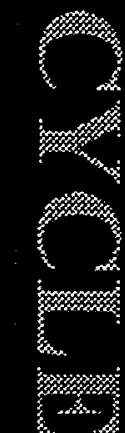

$3 \quad$

哆

移 


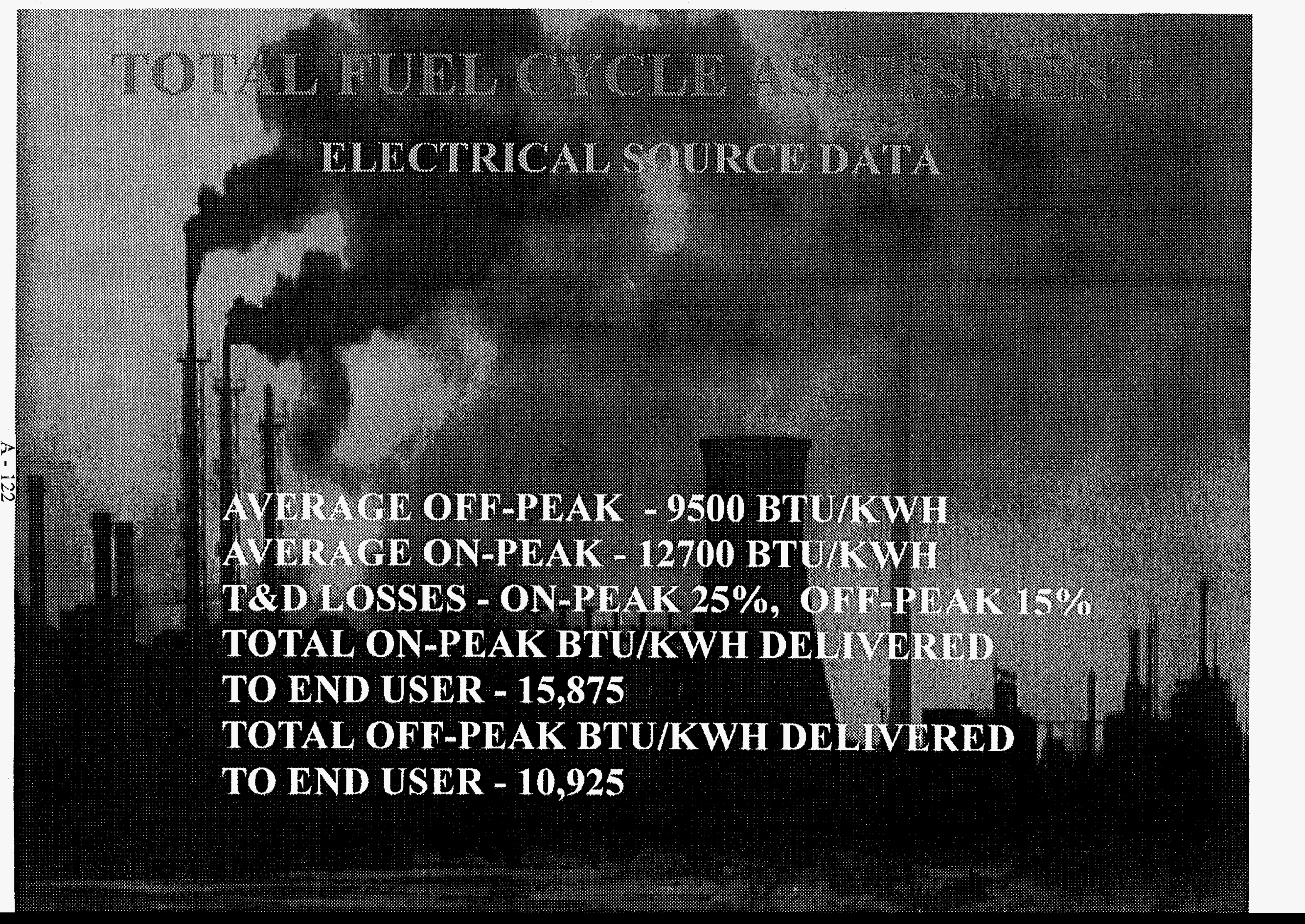


CFC countdown commences - the phase out could eliminate recent gains in energy savings

CFC compte commençe - la phase dehors a pu Eliminer gains récents dans le savings de l'énergie

Chlorofluorocarbons Countdown beginnt - die Phase, kōnnte aus neue Gewinne in Tatkraft Ersparnisse beseitigen

James L Block*, Christopher M Landry**

\begin{abstract}
This paper shall indicate the significant direct and indirect energy consumption impact potential of "CFC Abatement". In 112 weeks, CFCs--the refrigerants used to cool our air-conditioned buildings--will no longer be manufactured for use in the United States. Proven to destroy the ozone layer in the upper atmosphere, Chlorofluorocarbons (CFCs) and Hydrochlorofluorocarbons (HCFCs) also contribute to global warming and the resulting greenhouse effect. While the ban is generally welcomed, building owners and managers, as well as consumers, should anticipate that maintenance and energy costs will escalate considerably, as the ban, scheduled to go into effect in late 1995, will reduce the cooling capacity and efficiency of airconditioning systems. Solutions that range from use of the alternative refrigerants placed in existing equipment to new equipment using these same replacements will be costly. This cost is composed of very large capital expenditures, even larger operating costs, and if not carefully implemented, may eliminate the energy savings already accomplished through years of demand side management programs.
\end{abstract}

RESUME

Ce papier indiquera le significatif dirige potentiel de "CFC à et choc indirect de la consommation de l'énergie la Diminution". Dans 112 semaines, CFC--le refrigerants rafraichissait nos bâtiments climatisés-réfrigere pas de plus long est fabriqué pour l'usage aux Etas-Unis. A prouvé détruire la couche de (ozone] dans l'atmosphère supérieure, Chlorofluorocarbons (CFC) et Hydrochlorofluorocarbons (HCFC) aussi contribue à chauffage global et la serre résultante effectue. Pendant qu'on accueille le ban généralement, les propriétaires du bâtiment et les directeurs, les consommateurs de même que, doivent anticiper que l'entretien et l'énergie coûtent escalader considérablement, comme le ban, [scheduled] aller en l'effet dans tard 1995, réduira la capacité rafraîchissante et la compétence des systèmes de la climatisation. Les solutions que l'étendue de l'usage du refrigerants alternatif a mis dans exister l'équipement à nouvel équipement utiliser ce même [replacements] sera coûteux. Ce coût est composé de très grandes dépenses capital, égal plus grand opérer le coûts, et si pas soigneusement [implemented], peut éliminer déjà accompli à travers les ans des programmes de la direction de la côté de la demande au savings de l'énergie.

\title{
ZUSAMMENFASSUNG
}

Dieses Papier wird den bezeichnenden direkten und indirekten Tatkraftverbrauchzusammenprall Möglichkeit "Chlorofluorocarbonsverminderung zeigen". In 112 Wochen, Chlorofluorocarbons--hat der Refrigerants unsere klimatisiert Gebäude abgekühlt--will kein lang, wird für Gebrauch in den Vereinigte Staaten hergestellet. Hat die Ozone schicht in der oberen Atmosphäre, CFC (Chlorofluorocarbons) bewiesen zerstören, und HCFC (Hydrochlorofluorocarbons) auch trägt global bei, wärmen und die ergeben Gewächshauswirkung. Welle wird der Bann im allgemeinen bewillkommnt, Gebäudebesitzer und Leiter, wie gesünder wie Verbraucher, müssen wegnehmen, daß Aufrechterhaltung und Tatkraft Consider kosten steigern, wie hat der Bann, in Wirkung in spät 1995 angesetzt werden, wird den abkühlt Inhalt und Leistungsfähigkeit Anlagesystem verringern. Lösungen daß erstrecken Sie sich aus Gebrauch des alternativen Refrigerants, hat sich in bestehen Ausrüstung gesetzt, neuer Ausrüstung gebrauchen diesen selben Replacements, wird kostspielig sein. Dieser Preis wird sehr großer Kapitalausgabe zusammengesetzt, gleich groß operieren Preise, und haben wenn nicht vorsichtig durchgeführt, mögen der Tatkraft Ersparnisse schon vollendete fertige Jahre Forderungseiteverwaltungprogramme beseitigen.

* Director, Advanced Technologies, Burr Engineers, Inc., 3355 West Alabama, Suite 600, Houston, Texas 77098, USA

** Manager, Thermal Storage, Turbo Refrigeration Company, 1815 Shady Oaks Drive, Denton, Texas 76202-0396 USA 


\section{CFC Countdown Commences-The Phase-out Could Eliminate Recent Gains in Energy Savings.}

In 112 weeks, CFCs-the refrigerants used to cool our air-conditioned buildings-will no longer be manufactured for use in the United States. Proven to destroy the ozone layer in the upper atroosphere, Chlorofluorocarbons (CFCs) and Hydrochlorofluorocarbons (HCFCs) contribute to global warming; the result being the greenhouse effect. While the ban is generally welcomed, building owners and managers, as well as consumers, anticipate maintenance and energy costs will escalate considerably, as the ban, scheduled to go into effect in late 1995, will reduce the cooling capacity and efficiency of air-conditioning systems. Solutions are costly and range from the use of alternative refrigerants placed in existing equipment to new equipment using these same replecements. This cost is composed of very large capital expenditures, even larger operating costs, and if not carefully implemented, may eliminate the energy savings already accomplished through years of demand side management programs.

\section{Agreement Phases Out CFCs}

In the carly 1970's, a few scieatists began to be concenned about the depletion of ozone in the upper atmosphere, the gaseous layer that protects our planet from the harmful ultra-violet radiation of the sun. Their concerns about ozone depletion drew the attention of scientists world wide and in particular the United States National Aeronautics and Space Administration and the National Oceanic and Atmospheric Administration, whose follow-up studies discovered CFCs and HCFCs, both man-made chemicals that destroy ozone, present in the upper atmosphere. The studies also found these refrigerants contribute to global warming and act as greenbouse gases.

Based on these studies, an international meeting held in 1987 in Montreal, Canada, agreed to a slow but steady phase out of the use and production of CFCs (including R-11, R-12, R-113, R-114 and R-115) by the year 2000. "The Montreal Protocol " was signed by more than 70 nations. In 1990, the United States, the largest user of CFCs, pested the Clean Air Act, accelerating the phase out schedule of the original protocol. The in February of 1992, after an ozone "hole" was discovered over the northern hemisphere, and a "gouge" in the layer along the equator, President Bush accelerated the phase out to December 31, 1995. DuPont, a major supplier of CFCs, anounced it will stop manufacturing CFCs at the end of 1994. Action on the part of the United States is important because although containing only $5 \%$ of the world population, it contributes $30 \%$ of the 2.4 billion tons of CFCs released each year.

\section{Consequences of CFC Phase-Out}

Since CFCs and HCFCs were discovered to be harmful, attention has been focused on stopping the destruction of the ozone layer. Although many scientists and politicians called for an immodiate ban on the use of CFCs, scant attention has been paid to the consequences of such a ban. And there are many.

One major consequence will be a complete change in air-conditioning systems. When the ban on CFCs was first proposed, many people hoped technology would formulate new and safer alterratives. This hope has turved into 2 "wait and see" attinde towards the ban. This attitude, shared by 80 many, has brought us to a point of inevitable catastrophe. The catastrophe is this; 80,000 chillers is the United States use CFCs, current capacity to convert or replace these chillers to HCFCs can only produce 8,200 conversions and 4,800 replacements by January 1, 1996. Hence, 67,000 CFC laden chillers will remain with a very small supply of very expensive CFCs to serve them. Based upon "market" pressures the cost of chiller conversions and replacements may be "dictated" by manufacturers and service companies. Building owners and managers faced with the prospect of reduced or no cooling capacity, may be willing to pay nearly any price for CFCs, chiller conversions, or new equipment. These "market" pressures are already emerging. The days of cheap "Freon" (a trade name for refrigerants) are already over. The cost of a pound of Freon, any R-11, has recently gone from $\$ 2.00$ to $\$ 8.17$. Soon it may soar to $\$ 35.00 /$ pound and higher as the deadline approaches and with implementation of tariffs, taxes, and handling/processing costs.

In fact, it is the handling of CFCs and HCFCs-rather than the use-that has changed most dramatically. They can no longer be vented to the atmosphere; if done, the fine is $\$ 25,000$ (effective July, 1992). The United States Environmental Protection Agency (EPA) is aggressively pursuing violators and has already levied hundreds of thousands of dollars in fines. Specific criteria have been established to capture these refrigerants in an effort to reduce emissions due to maintenance and demolition procedures. Also, it is important to note that all reciprocating chillers, split system a/c systems (like in your home), and small packaged equipment use R-22, an HCFC which has been targeted for accelerated phased out near the end of this century. All automobile air conditioners use R-12 which will be phesed out 
in 1995. The U.S. Environmental Protection Agency (EPA) has placed CFCs on its list of toxic hazards. The handling of equipment parts and related lubrication oils contaminated by CFCs is similar to that of asbestos abatement.

\section{Systems To Change}

As CFCs and the HCFC R-22 are phased out, building cooling systems (chillers, rooftop-units, beat pumps, water coolers, ice makers, refrigerators, etc.) will need to be either upgraded with new, acceptable refrigerants, or be replaced with non-CFC cooling systems.

\section{Upgrading Chillers}

Upgrading a cooling system with new refrigerants such is R-123, however, can cause a loss in a cooling system's capacity and will necessitate costly chiller modifications. For example, simply replacing R-11 (a CFC used in $90 \%$ of all chiller equipment) with R-123 can cause a loss in capacity of 5-40\%, based upon the exact configuration of the individual unit, and can increase eaergy consumption by $1-40 \%$.

In upgrading to R-123, two options exist: first is chiller modification for simple compatibility; i.e. gaskets, seals, and motor windings due to the corrosive nature of R-123. Currently the cost is approximately $\$ 100$ per ton of chiller capacity. This "minimal" conversion, referred to as an "up-grade", has documented capacity losses of some 30-40\% while maintaining the original energy consumption. It is important to note that the time frame from such a modification is a few days to week.

The second option is total modification which in addition to those changes needed for compatibility may include impeller, tube, motor and/or gear changes to regain some of the lost chiller capacity and rocapture efficiency losses. Curreatly this "maximized" modification costs approximately $\$ 150-\$ 250$ per ton. This option, although it can recapture most of the losses, takes several months to complete. In either option, energy costs and consumption may increase by $5 \%$ to $40 \%$ based upon the amount of time and/or money spent on the conversion. The time needed to conserve energy may simply not be available.

These two options were recently presented to a building owner. The project consisted of two 1,000 ton chillers with an efficiency of $.75 \mathrm{Kw} / \mathrm{ton}$ maintaining an 1,100 ton cooling load in a partially oceupied building. Together the partially loaded chillers consumed a total of $824 \mathrm{Kw}$ of peak demand. The project engineer together with chiller manufacturer made detailed equipment calculations converting the chillers to R-123. Under the "up-grade" scenario, each chiller de-rated to 600 tons consuming $650 \mathrm{Kw}$ each for a new demend peak of $1,300 \mathrm{Kw}$, would cost $\$ 200,000$, and take only a few days to complete. The "maximized" option would de-sate the units to 921 tons each, improve the efficiency to $.69 \mathrm{Kw} / \mathrm{ton}$, cost $\$ 600,000$, and would need to be phased over three months. The edditional $\$ 400,000$ capital investment was estimated to be recaptured or "paid back" in just under 2 years. The building owner when presented with these options stated "electrical costs are passed through to the tenants by the lease agreement, capital costs for the building are not" and proceeded with the least costly "up-grade" option. Please note that it is unlikely all building owners will take such a stance, but many may be forced into such a decision by the lack of either time or funds.

Recently, a chiller manufacturer, in an effort to place some perspective on the magnitude of the CFC abatement problem, estimated that these 80,000 chillers in the United States average 1,000 tons each in capacity. Using this data let us make a simplified calculation. First, assume each chiller has an average efficiency of $.75 \mathrm{Kw}$ /ton. Applying - 10\% loss in efficiency would add some $6,000,000 \mathrm{Kw}$ in electrical demand. Annual energy consumption could increase by some $1,950,000,000,000 \mathrm{Kwh}$. This could add 84,000 tons of $\mathrm{CO}_{3}$ to the atmosphere.

\section{Replacing Chillers}

An alternative to upgrading is total system replacement. It requires a significant capital expenditure of approximately $\$ 500$ per ton for the chiller alone. However, the new chiller equipment curreatly available is in many cases far less efficient than the equipment now in use due to the lower thermodynamic efficiency of the replacement refrigerants. Again a loss in energy efficieacy and an increase in operating costs. 


\section{Thermal Stonage}

Perhaps the most promising solution to all these problems is Thermal Storage Technology, which employs stored energy for cooling. Cooling energy can be stored in large water tanks, or in the form of ice, or in containers filled with a water/salt solution. Thermal Storage takes advantage of the difference between peak daytime and off-peak nighttime cooling hours. This differeoce results into smaller cooling plants, which mean lower refrigerant use-in some applications, up to $80 \%$ less. Thermal Storage can abift the entire chiller operation to night hours when ambient temperatures are lower and some of the efficiency losses can be rocovered. The addition of other system "enhancements" can save tremendous amount of exergy.

The cost of chiller modification (including those required for CFC abatemeat), coupled with the cost of the thermal storage modium, can result in a total capital investment peyback of three years or less. The use of thermal storage designed with energy conservation "enhancements" offers additional opportunities to save energy and operating costs in other air-conditioning related equipment, both inside and outside the central plant. A roduction in operating cost of 30-50\% and a 25\% reduction in actual energy consumption, compared to the old air-conditioning energy costs and energy usage, is possible.

Of all possible solutions, Thermal Storage can be the least expensive capital expenditure, achieve a two to three year payback on that expenditure, and consume far less energy than conventional solutions.

\section{System Efficiencies}

Conventional verses Thermal Storage system efficiencies, is perhaps the most hotly debated issue within the engineering community, even without the potential losses associated with CFC abatement. Thermal Storage has traditionally been portrayed as an energy inefficient system. In fact, many applications to date appear to use more energy because, historically. Thermal Storage has always been seen as a demand shifting measure. Due to the lower evaporator temperatures required by many Thermal Storage chillers, early laboratory tests and refrigeration theory indicated more energy was required. Designers assuming en inherent energy loss spent little design time optimizing energy conservation. However, these laboratory tests only consider a single variable in a complex and dynamic system. One must examine the issue from a much broader "systemic" viewpoint. For example, few designers or researchers have considered the fact that generation and distribution of electricity has system losses in the 50 to $70 \%$ range. Simply stated the conservation of one kilowatt of electricity at the building is equal to four kilowatts of energy savings back at the generator. The time of day "when" electricity is used it must also be factored into the equation. Energy losses vary from day to night usage. Research indicates a kilowalt-hour (Kwh) consumed during daylight hours consumes 12,700 btu's. This same Kwh consumed at night only requires 9,500 btu's, a $25 \%$ reduction. In addition, transmission and distribution losses, typically 8-15\% on heavily loaded wires and transformers during the heat of the day, are 2-5\% lower at night when ambient temperatures are lower and these same wires and transformers are lightly loaded. Overall, when analyzing the energy consumption "systemically" from source to ead use, "conventional high efficiency systems" operating under actual conditions and applications consume far more exergy when compared to Thermal Storage systems "enhanced" with conservation messures.

Simply installing "high" efficiency pieces of HVAC equipment in \& building does not guarantee energy efficiency. For example, the vast majority of HVAC systems operate in a part load condition. That part load is usually in the 50 to 70\% range as a seasonal average. A "high efficiency" chiller $60 \%$ loaded may in theory be operating at $.65 \mathrm{kw} / \mathrm{ton}$, however, the parasitic equipment (condenser pumps and tower) can not modulate effectively enough to procisely track the load variations. The actual part load efficiency of the "cooling plant" can reach well over $1.5 \mathrm{kw} / \mathrm{ton}$. Graph-1 indicates the actual annusal energy consumption of the entire cooling system in a building that won an ASHRAE energy design award. As the data indicates, at lower cooling loads, the part load efficiency for the entire HVAC aystem can reach 3-4 kw/ton. Thermal Storage in a full shift strategy disassociates the load profile from the chiller plant. The electric "ice" chiller runs at $.9 \mathrm{kw} / \mathrm{ton}$ and then shuts off when the storage tank is full. A direct comparison of the seasonal part load value of the two plants would show the conventional system averaging 1.3-1.6 kw/ton, whereas, the Thermal Storage plant is in the $.7-9 \mathrm{kw} / \mathrm{ton}$ range.

From the standpoint of resource energy conservation, the Thermal Storage chiller does not incur the distribution losses of the daytime electrical grid and is, in fact, more efficient than any other cooling system. Additionally, if all energy saving attributes associated and possible with each system are analyzed, enhanced Thermal Storage is again, clearly more energy efficient. 


\section{Methodology "A Sample Proiect"}

To demonstrate the source of these potential windfall eaergy losses or savings, we will examine a specific project that has been constructed and has known energy consumptions. We shall substitute various alternatives including an "Alternative Fuels" Thermal Storage plant for the electrically driven plant that was constructed and model the impacts with the aid of a computer simulation program. The analysis shall also include a classic ASFRAE "High Efficiency" design using various refrigerants that meets all the parameters set by ASHRAE Standard 90.1 (1992), a United States efficiency standard, which shall be referred to as conventional and the actual Enhanced Thermal Storage system using electrically driven chillers.

Our sample project is a 325,000 square foot office building locatod in Houston, Texas. The owner is a speculative developer so capital cost is most important to him. The tenent, however, is a large engineering firm whose main interests are reliability and long term energy cost savings. Often these two points of view are envisioned as mutually exclusive, but in this case a compromise was reached after oxtensive analysis. Based upon the tenents reeds, the project cooling load calculation parameters are as follows;

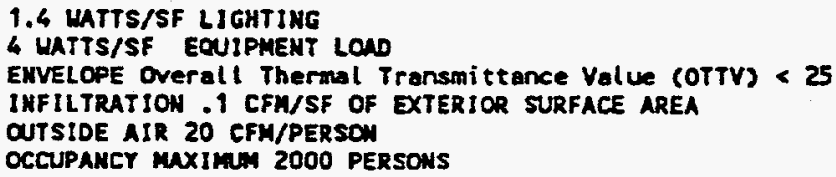

A computer simulation of project cooling loods resulted in the following:

$\begin{array}{lll}\text { System } & \text { Cooling Lood } & \text { Supply Air } \\ \text { Conventional } & 940 \text { tons } & 524,000 \text { CFM } \\ \text { Enhanced Thermal Storage } & 726 \text { tons } & 208,000 \text { CFM }\end{array}$

The application of the various design approaches is centered around the attributes or strengths and weaknesses of each system and the institutionalized desiga practices associated with each system. The key wording here is design attributes. Referring to our sample project, consider this comparison of desiga strategies that produced the variance in cooling load.

\section{Air Distribution}

Conventional variable air volume (VAV) air systems are designed for a cooling coil discharge air temperature of $55^{\circ} \mathrm{F}$ at a room temperature setpoint of $75^{\circ} \mathrm{F}$. Fresh air, even under the new ASHRAE standards, is $8.5 \%$ of the total supply air. Because of the amount of air required to cool the space by this design (a $20^{\circ}$ differential temperature or delta "T"), the limited ceiling space imposed by the shortest possible floor-to-floor height of the project (an architectural capital cost savings technique), coordination of fire sprinkler piping and clearance for lighting fixtures, large and heavy rectangular duct with a poor aspect ratio is required. This translates into the single most costly item in the HVAC system for both energy consumption and capital cost. Duct friction loses are bigh because of the high velocities and poor aspect ratios needed to fit the duct above the ceiling. To limit mechanical room space (a architectural requirement to maximize net rentable space) and equipmeat capital cost, the air bandling units (AHUs) are selected at 550 FPM velocities across the coils and filters. Overall this equates to some 4.0" of total static pressure on the fin and some 600 horsepower of fan motors for the sample project. Additionally, recent concern for improved indoor air quality are pushing designers into using constant volume fan powered terminal units (Series Fan Powered VAV boxes) in conventional VAV air distribution systems to eliminate stagnant air problems associated with early VAV applications. This adds capital cost and $112 \mathrm{HP}$ of fractional, inefficient fan motors to the conventional system.

Conversely, the eahanced Thermal Storage system is designed to deliver $45^{\circ} \mathrm{F}$ air to the space, but only at peak the time of cooling load, at less than peak the air temperature is reset upwards. This approach dramatically reduces the required volume of air circulated but does not adversely affect indoor air quality, minimum air circulation is .64 CFM per square foot, three times the ASHRAE minimum standard of .2 CFM per square foot. Fresh xir is now $21 \%$ of the total supply air. The primary duct system (duct between the AHU and the fan powered box) is sized for a 35 F temperature delta at the time of peak cooling load requirements. During part load conditions the cooling coil leaving air temperature is reset upward instead of varying the system volume. Constant volume fan powered terminals are used to mix the 
primary supply air (at a temperaturo which ranges from 40F to 60\%) with recirculated plenum air to maintain the space temperature setpoint. This design approach reduces the primary and socondary (duct between the fan powered terminal unit and the space) air volume to some 50\% of the conventiond system. Designing for the maximum amount of air at the lowest temperahure at the load peak allows for much smaller round duct to be used. Round duct is much more cost effective and hes less friction loss due to the perfect aspect ratio. The difference in air volume combined with the perfect aspect ratio translates into a duct aystem savings in sheetwetal poundage, associated insulation, and other air systems devices of some $70 \%$. The enhanced apprasch is to again "give back" some of these capital cost savings in order to reduce on site energy consumption. The duct system is slightly oversized with lower velocities in order to significantly reduce total static pressure. Additionally, because the AHU's are $50 \%$ smaller, they may be economically changed to face velocities of less than 300 FPM. Overall this equates to a system with less then $1^{\circ}$ total static pressure including the series fan powered terminal units. Ductwort for the project is reduced by some 100,000 pounds of galvanized sheetmetal. Air handling unit fan motor size for the entire project is reduced to $90 \mathrm{HP}$ and fan powered boxes use 56 HP. The smaller fan motors result in a reduction of 160 tons of cooling. This greatly exceeds the additional tonnage due to the excess latent load from producing 45० $\mathrm{F}$ air on a "design" day peak hour. Remember AHU leaving the air temperature is reset upwards at non-peak loads which greatly reduces the excess latent load. Additionally, because of the lower relative humidity of the cooler air the space thermostat can be reset upuard to $78^{\circ} \mathrm{F}$ on a peak day further reducing the required cooling load.

\section{Water Distribution}

Consider the differences in design of the chilled water systems for both the conventional and enhanced Thermal Storage systems. The traditional conventional chilled water system is designed based upon $45^{\circ} \mathrm{F}$ leaving water temperature (LWT) and $55^{\circ} \mathrm{F}$ entering water temperature (EWT) at the chiller. This ten degree temperature differential causes large volumes of chilled water to be circulated. This large volume translates into very large piping sizes. To minimize capital cost designers select pipe sizes at high velocities. This volume and velocity of water dictate a system pressure loss of 100' in the sample project. The "eshanced" Thermal Storage chilled water system design takes a different approach. The chilled water system is sized based upon $36^{\circ} \mathrm{F}$ LWT and $60^{\circ} \mathrm{F}$ EWT at the chiller and very low velocities when sizing piping. The lower volume of water caused by the twenty-four degree temperature differential significantly reduces pipe size and therefore capital cost. The enhanced Thermal Storage approach is to use a lower velocity to reduce the system head pressure and "give back" some of the capital cost savings. This becomes operationslly attrective because of the vastly smaller pump sizes coupled with moderately smaller piping. The overall effect is a $25 \%$ smaller chilled water distribution system with $75 \%$ smaller pumps.

\section{Chillers, Cooline Tower, and Condenser Pumps}

A comparison of central plants between the conventional and enhanced Thermal Storage (ETS), both electric and natural gas, finds they are designed from completely different perspectives. The conventional plant is designed besed upon a single theoretical "peak" that occurs only 1 percent of the time during annual building operation. Conservative designs would dictate three 325 ton .665 Xw/ton R-123 based chillers. In fact, this sizing was requested by the owner. Cooling towers and condenser water pumps are selected at $3 \mathrm{gpm} / \mathrm{ton}$ and are quite large as is the inter-connoctive piping. Three $75 \mathrm{Hp}$ fans for the towers and three $40 \mathrm{HP}$ pumps. This equates to a peak electrical demand of 995 $\mathrm{Kw} / \mathrm{ton}$ for the plant. However, the efficiency of the plant at peak tonage ( $940 \mathrm{tons})$ is $1.04 \mathrm{Kw} / \mathrm{ton}$. The part-load efficiency reaches $1.8 \mathrm{Kw} / \mathrm{ton}$ during minimum load. The average anoual efficiency is $1.5 \mathrm{Kw} / \mathrm{ton}$.

The electrically driven ETS plant is quite different. Two 200 ton "ice" chillers were selected using a "Daily Shift" operating strategy. The efficiency of these cbillers is $.75 \mathrm{Kw} / \mathrm{ton}$ while making ice in the cooler nighttime ambient temperatures. Again selecting the towers end pumps at $3 \mathrm{gpm} /$ ton wo now have two $30 \mathrm{HP}$ tower fans and two $10 \mathrm{HP}$ pumps. The tower reduction is due to lower nighttime ambient in addition to the tonnage reduction. The pump reduction is due to the "enhanced" approsch of upsizing piping slightly to reduce system operating pressure. This results in a central plant efficiency of $.95 \mathrm{Kw} / \mathrm{ton}$. The average annul efficieacy actually increases to $.80 \mathrm{Kw} / \mathrm{ton} 2 \mathrm{~s}$ nighttime temperatures drop in fall, winter, and spring.

The natural gas engine driven "alternate fuels" chiller was selected using a "weekdy load leveling" strategy. This equates into two 100 ton ice chillers. Selecting the towers and pumps at $4 \mathrm{gpm} /$ ton due to the engine heat we now bave two $30 \mathrm{HP}$ tower fans and two $10 \mathrm{HP}$ pumps. 
As discussed eartier nighttime operation and lower ambient temperatures this is not simply an observation, but when analyzed a distinct design advantage for the Thermal Storage system. Although the Thermal Storage chiller is theoretically less efficient because of the lower suction temperatures required, in this application the chillers were selected with vastly larger evaporator and condenser sections. In this approach the ETS system can take better advantage of these lower ambient. Consider that to maintain compressor head pressure for adequate oil pressure and refrigerant "lift" a conventional chiller requires a pressure differential equivalent to spproximately $27^{\circ} \mathrm{F}$ sbove the suction temperatere. At a suction temperature of $35^{\circ}$ (to maintain $45^{\circ}$ LWT) the minimum setpoint would be $62^{\circ} F$, bowever, most controls contractors set this up to $80^{\circ} \mathrm{F}$ for a safety factor. An ice chiller operates at $22^{\circ} \mathrm{F}$ (to make ice at $32^{\circ} \mathrm{F}$ ), therefore, the minimum operating temperiture is approximately $49^{\circ} \mathrm{F}$. These factors combine to redefine the operating efficiency algorithm. This redefinition considerably closes the gap in efficiency. The application of these design tochniques resuits in actual construction cost eatimates and computerized energy use simulations as follows:

\begin{tabular}{|c|c|c|c|}
\hline 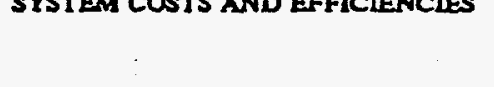 & $\begin{array}{l}\text { ALTERNATTVE FUELS ETS } \\
\text { (726 LORE) }\end{array}$ & $\begin{array}{l}\text { CONVENTIONAL } \\
\text { (940 wn) }\end{array}$ & $\begin{array}{l}\text { ELECTRIC ETS } \\
\text { (726 tOns) }\end{array}$ \\
\hline DNTALLED COST & $\$ 6.75 / S F$ & \$9.81/SF & $\$ 7.02 / S F$ \\
\hline SYSTEM EFFICIENCIES & KW/TON OF PEAK LOAD & KWTTON OF PEAK LOAD & KW/TON OF PEAK LOAD \\
\hline $\begin{array}{r}\text { PART } 1 \text { CENTRAL PLANT } \\
\text { CHIILER }\end{array}$ & $\mathbf{N} / \mathbf{A}$ & .665 (R-123) & $\begin{array}{l}.75 \text { ICE MAKDNG \& } 59 \text { IN } \\
\text { CHIILRR MODE. (R-134a) }\end{array}$ \\
\hline TOWER & .3 & .19 & .08 \\
\hline $\begin{array}{l}\text { PUMPS } \\
\text { PART } 2 \text { WATER SIDE }\end{array}$ & .1 & .12 & .027 \\
\hline PART 3 AR SWE & .04 & .08 & .04 \\
\hline $\begin{array}{l}\text { AHU'S } \\
\text { FP BOXES }\end{array}$ & .201 & $\begin{array}{l}.638 \\
.119\end{array}$ & $\begin{array}{l}.201 \\
.07\end{array}$ \\
\hline $\begin{array}{l}\text { PART \& CONTROLS } \\
\text { CPU }\end{array}$ & & & \\
\hline $\begin{array}{l}\text { TOTALS } \\
\text { ON-PEAK TOTALS }\end{array}$ & $\begin{array}{l}.0004 \\
.714 \\
.714\end{array}$ & $\begin{array}{l}.0004 \\
1.8124 \\
1.82124\end{array}$ & $\begin{array}{l}.0004 \\
1.172 \\
0.311\end{array}$ \\
\hline $\begin{array}{l}\text { ANNUAI KWH } \\
\text { KWH PREMTUM }\end{array}$ & $\begin{array}{l}1,785,865 \\
0\end{array}$ & $\begin{array}{l}5,890,315 \\
4,104,450\end{array}$ & $\begin{array}{l}2,925,750 \\
1,139,005\end{array}$ \\
\hline ANNUAL FUEL CONSUMPTION (SCFI & $4,383,000$ & & \\
\hline $\begin{array}{l}\text { End Ues Inteneity (KBTU/SF/YEAR) } \\
\text { Source Energy Utilization }\end{array}$ & 32.77 & 61.25 & 30.72 \\
\hline (KETU/SE/YEAR) & 83.81 & 230.17 & 85.52 \\
\hline EMISSIONS (TONS OF (Q) & 3930 & 8393 & 4169 \\
\hline ANNUAL FUEL COST & $\$ 14,463$ & & \\
\hline $\begin{array}{l}\text { TOTAL ANNUAL OPERATING COST } \\
\text { PERCENT SAVINGS }\end{array}$ & 5139,473 & 5412,322 & $\begin{array}{l}5125,807 \\
70 \%\end{array}$ \\
\hline $\begin{array}{l}\text { WATER USAGE } \\
20 \text { YEAR LIFE CYCLE SAVINGS }\end{array}$ & $\begin{array}{l}291,000 \text { GAls } \\
\$ 5,729, \$ 29\end{array}$ & $\begin{array}{l}218,000 \text { GALS/YEAR } \\
\text { SO }\end{array}$ & $\begin{array}{l}170,340 \text { GALSTYEAR } \\
\$ 6,016,815\end{array}$ \\
\hline LBS OF REFRIGERANT REQUIRED & 520 & 1950 & 1040 \\
\hline ALLOWABLE REFRIO. LOSSTYR & 78 & 292 & 156 \\
\hline
\end{tabular}

The End User Intensity (EUT) would indicate the electric enhenced Thermal Storage system to be the most energy efficient. However, this calculation does not account for generation and distribution losses is the electrical grid. The source energy utilization figures do account for these losses. Based upon these figures the alternative fuels eahanced Thermal Storage system is clearly the most energy officient, however, this system does use more water than any other system. Furthermore, the carbon dioxide emissions savings are equally dramatic.

When analyzing energy consumption from 2 "systemic" viewpoint demand and KWH savings such as these will belp defer power plant construction, electrical distribution grid upgrades, and ultimately electrical rate increases. This should make professional and lay conservationists happy because fewer power plants mean less "greenhouse" emissions. Additionally, more electrical power used at night results in less $\mathrm{CO}_{2}$ and $\mathrm{SO}_{2}$ due to the increased prime mover 
efficiency through lower ambient temperatures entering the unit. As indicated previously Thermal Storage systems operate at night when temperatures are lower. These lower temperatures mean greater chiller efficiency and less water loss for water cooled units. Less cooling tower evaporation means less chemical usage. This should also equate to lower maintenance and operational costs due to less chemical use, which equates less toxic waste.

But what does all this discussion of a new building have to do with CFC abatement? The answer is that in understanding how enhanced Thermal Storage operates, how much it costs, and areas of potential energy savings, the design strategies can be applied to existing buildings as CFCs are phused out.

\section{Retrofit Proiects}

CFCs will become more scarce very quickly over the near future. The "Montreal Protocol" allows for $25 \%$ of the 1986 CFC production levels to be manufactured in the closing years of the ban. The United States EPA has mandated only $15 \%$ of that quantity be produced. The shortfall in CFCs is very real and is quietly stalking an unaware business community.

As these 80,000 existing chillers are converted or replaced the vast majority will consume more energy due to the capacity/efficiency losses previously indicated or will simply run out of refrigerant and stop functioning. Theoretically with "maximized" chiller modifications this need not be true, however, practically, the lack of both time and capital will result in these losses as owners opt for the faster cheaper HCFC "up-grades". Building owners may find themselves unable to keep some of their chillers in operation. In this scenario Thermal Storage can also help. Thermal Storage design strategies spread the cooling load, usually concentrated in the daylight hours, over many more hours, therefore, the required chiller size is smaller. Consider that a building with a peak load of say 2000 tons using two 1000 ton chillers would need only one unit with the addition of a storage medium. Refrigerant from the other chiller could be captured and saved for future use. This would expand the window of time needed to replace or retrofit the chiller plant while keeping the building in full operation. Proper forethought and detailed construction phasing can implement the utilization of Thermal Storage independently of chiller manufacturer timeliness.

Utilizing Thermal Storage and applying the eabancements such as cooler air and water to existing ductwork and piping can result in 40-50\% fan and pump evergy reductions. This is possible because as less air and water are circulated to meet the same loads, again due to the lower temperanures available, the existing ductwork and piping become oversized and friction loss is reduced. The combination of less quantity and lower friction losses save tremendous aroounts of energy. Recent research has shown that fan and pump energy consumption may in many cases exceed that of the chiller plant. The study of the results of several installations using cooler air and water has indicated a reduction of .5 to .75 watts per square foot in demand and 15-25\% of total annual Kwh consumption.

Retrofit applications of Thermal Storage technologies need not be limited to facilities with chilled water plants. Facilities utilizing an HVAC system with an EER of (13) thirteen or less are a viable candidate. This includes; roof-top systems, split-system DX, air-to-air heat pumps, water source heat pumps, air cooled chillers, and many other configurations of cooling systems. Prospective candidates need not have a narrow occupied time period, (24) twentyfour hour facilities qualify. Size is also not an issue Projects as amall as 1000 SF (small churches in the south have used an ice-on-pipe design for some 30 years sized to build ice over 164 hours to be used on Sunday mornings) and as large as an entire city can cost effectively utilize Thermal Storage technologies.

\section{Summary}

As the production of CFCs are phased out by 1995, and HCFCs by 2015 (R-22 by 2005 ), \& building's operating budget will be adversely affected. Loss of chiller capacity, higher energy costs, higher ownership and operating costs, maintenance costs, and possibly new toxjc waste criteria, will result. As existing systems begin to consume more energy, new construction energy use can be "minimized" using the techniques indicated. On site energy use can be reduced by upwards of $70 \%$ as compared to current design practices. Additionally, the reduction in construction materials from the use of Thermal Storage "enhancements" may save many times that of the on-site energy use over the life of the building. For example, in a study due out in early 1994 the energy consumption to produce one pound of installed galvanized ductwork is estimated to be some 1,000,000,000 btu's starting with the raw iron ore and eading with installation, including all the "externallities" in between. Based upon the 100,000 pounds of ductwork saved in 
our example project that equates to some $100,000,000,000,000$ btu's of energy in only one of bundreds of construction materials. The 20 year life cycle energy savings of the electrically driven Thermal Storage system equates to only $86,640,000,000$ bu's of energy. Energy conservation must be examined from a "systemic" viewpoint. Thermal Storage is the only proven technology available today that can save energy thought; the reduction of construction materials, the reduction of losses in the electrical generation/distribution network itself, the reduction of on-site energy use, reduce the amount of refrigeration gases required for cooling and the associated leakage, reduce power plant emissions, and pay for itself by minimizing capital investonent and maximizing operating cost savings.

\section{REFERENCES}

ASHRAE 1985. ASHRAE Tectnical Data Bullerin: Thermal Storage. Atlanta: American Society of Heating, Refrigerating, and Air-Conditioning Engineers, Inc., Jan 1985.

ASHRAE 1989. ASHRAE Technical Data Bulletin; Cool Storage Applications. Volume 5. Number 3. Atlanta: American Society of Heating, Refrigerating, and Air-Conditioning Engineers, Ioc.

ASHRAE 1989. ASHRAE Technical Data Bulletin: Cool Storage Madeling and Design. Volume 5, Number 4. Atlanta: American Socjety of Heating, Refrigerating, and Air-Conditioning Engineers, Inc.

Dorgan, C.E. and J.S. Elleson. 1988. Cold air distribution design guide. EPRI EM-5730, Mar. 1988

Block, J.L. and Landry C. 1989. Cost Effective Ice Storage for Hot Climates. ASHRAE Transactions Far East Conference on Air Conditioning in Hot Climates Kuala Lumpur, Malaysia, Oct 25-28, 1989

Block, J.L. and Landry C. 1991. Global Warming and Thermal Energy Storage. ASHRAE Transactions Far East Conference on Environmental Quality Hong Kong, Nov 5-8, 1991

Scofield C.M. 1991. Low-Temperature Air with High IAQ. ASHRAE Transactions Far East Conference on Environmental Quality Hong Kong, Nov 5-8, 1991

Block, J.L. 1992. Enhanced Thermal Storage that Saves Energy. ACEEE Proceedings 1992 Summer Study on Energy Efficiency in Buildings Commercial Technologies: Design and Operation Book 1

Mahoney T.A. 1993. Slow Conversion to Non-CFCs Worries Chiller Manufacturers. The Air-Conditioning, Heating and Refrigeration News, April 1993. pp 3.

Wendland R. and Blatt M. 1992. Reliable and Efficient: Cool Storage Meets the Challenges of the '90s. The Electricity Journal. Volume 5 Number 10

Block, J.L. 1993. A Study of Construction Materials Energy Externallities. Fuel Cycle Analysis Conference. Austin Texas.

Block, J.L. 1992. Alternative Fuels Enhanced Thermal Storage That Saves Energy. Proceedings Governors Energy Conference. Austin Texas. Oct. 1992. 


\section{GRAPH-1 AVERAGE DAILY KW/TON}

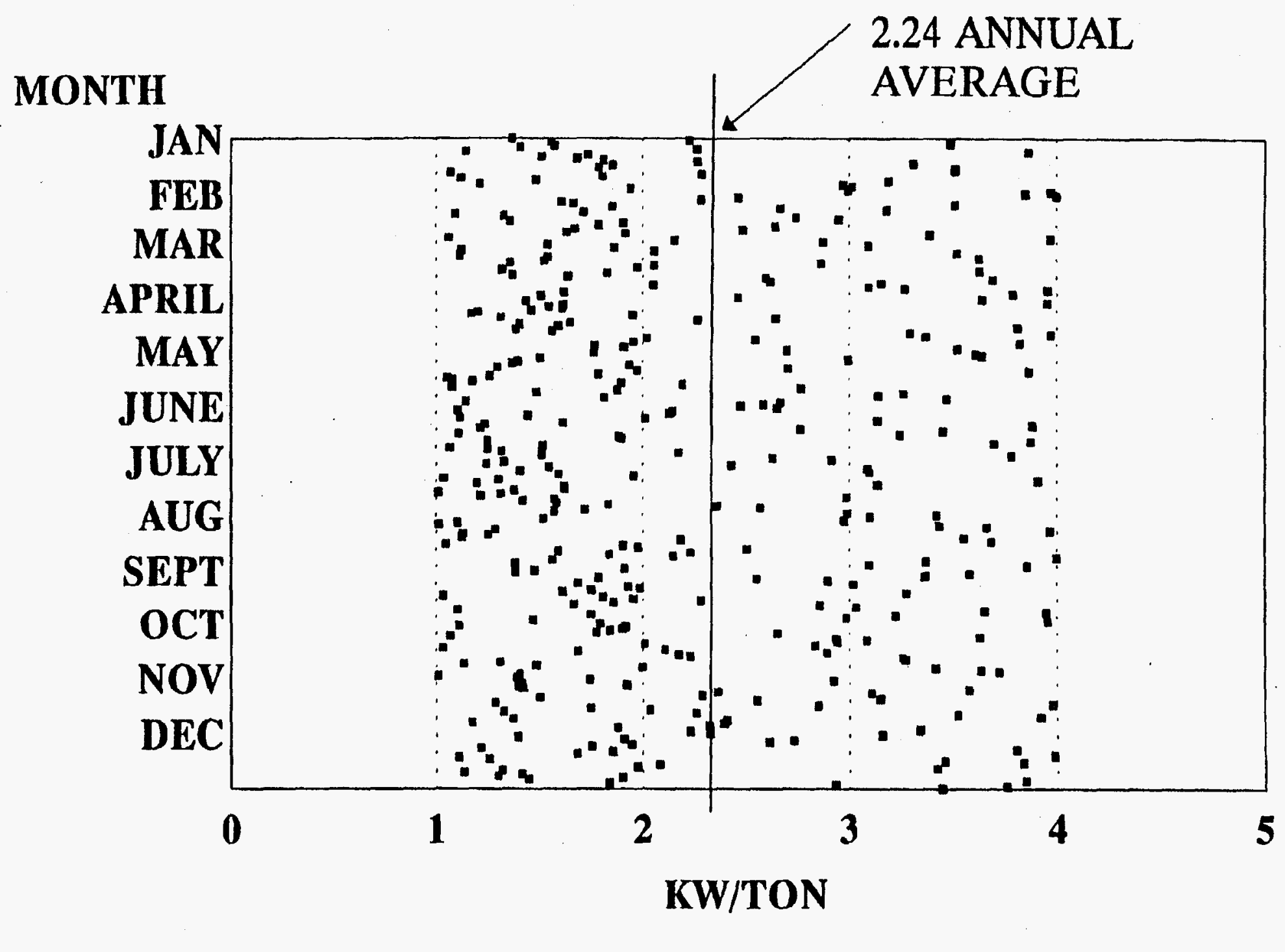

NOTE: VALUES INCLUDE ALL HVAC SYSTEM EQUIPMENT

ज. 
UTILITY APPLICATIONS OF TOTAL FUEL CYCLE ANALYSIS IN PLANNING 
A -134 


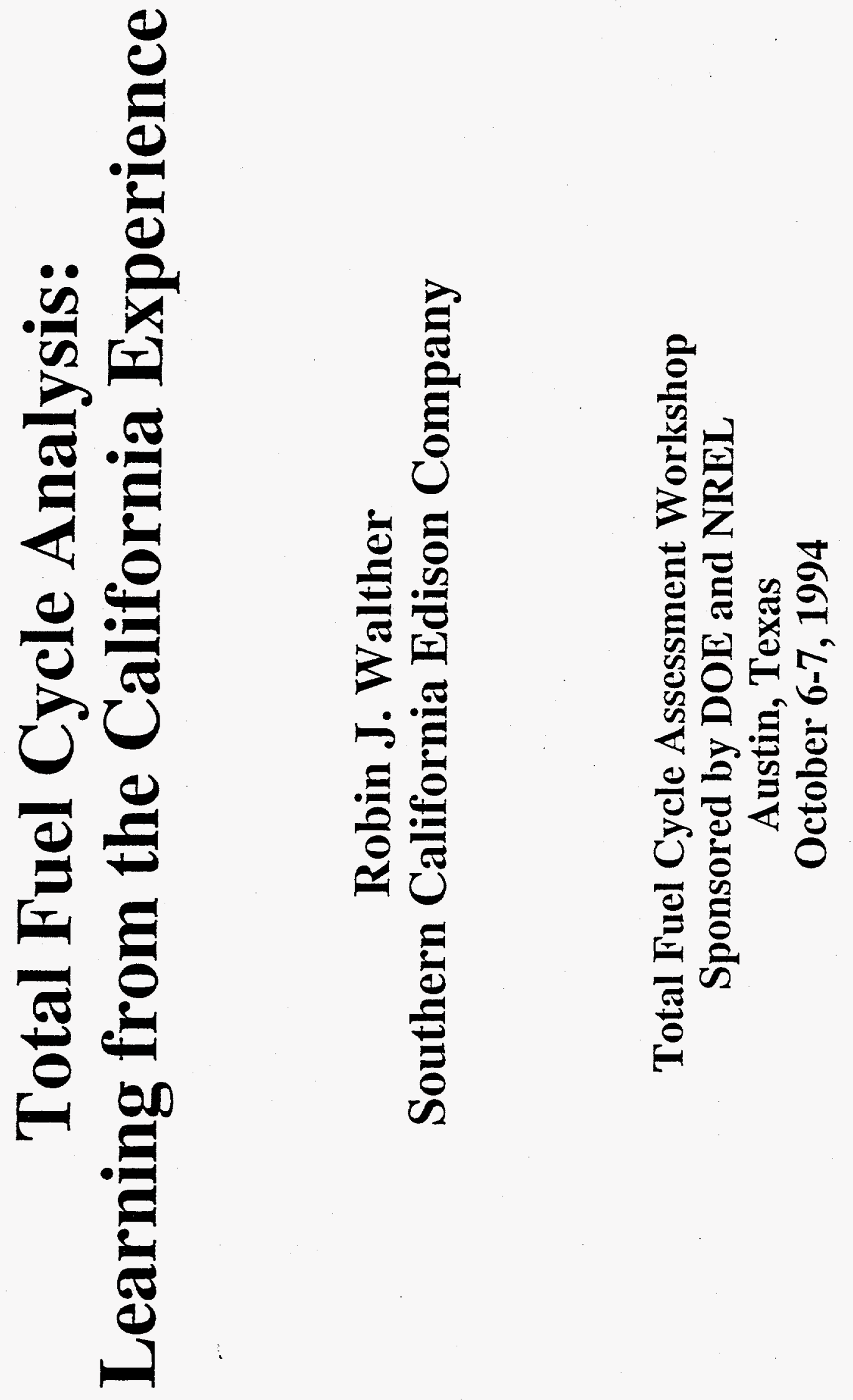




\section{Outline}

- Southern California Edison

- Experience with Environmental Externalities

- Possible Implications of Utility Restructuring

- Lessons Learned 


\section{Southern California Edison - Key 1993 Statistics}

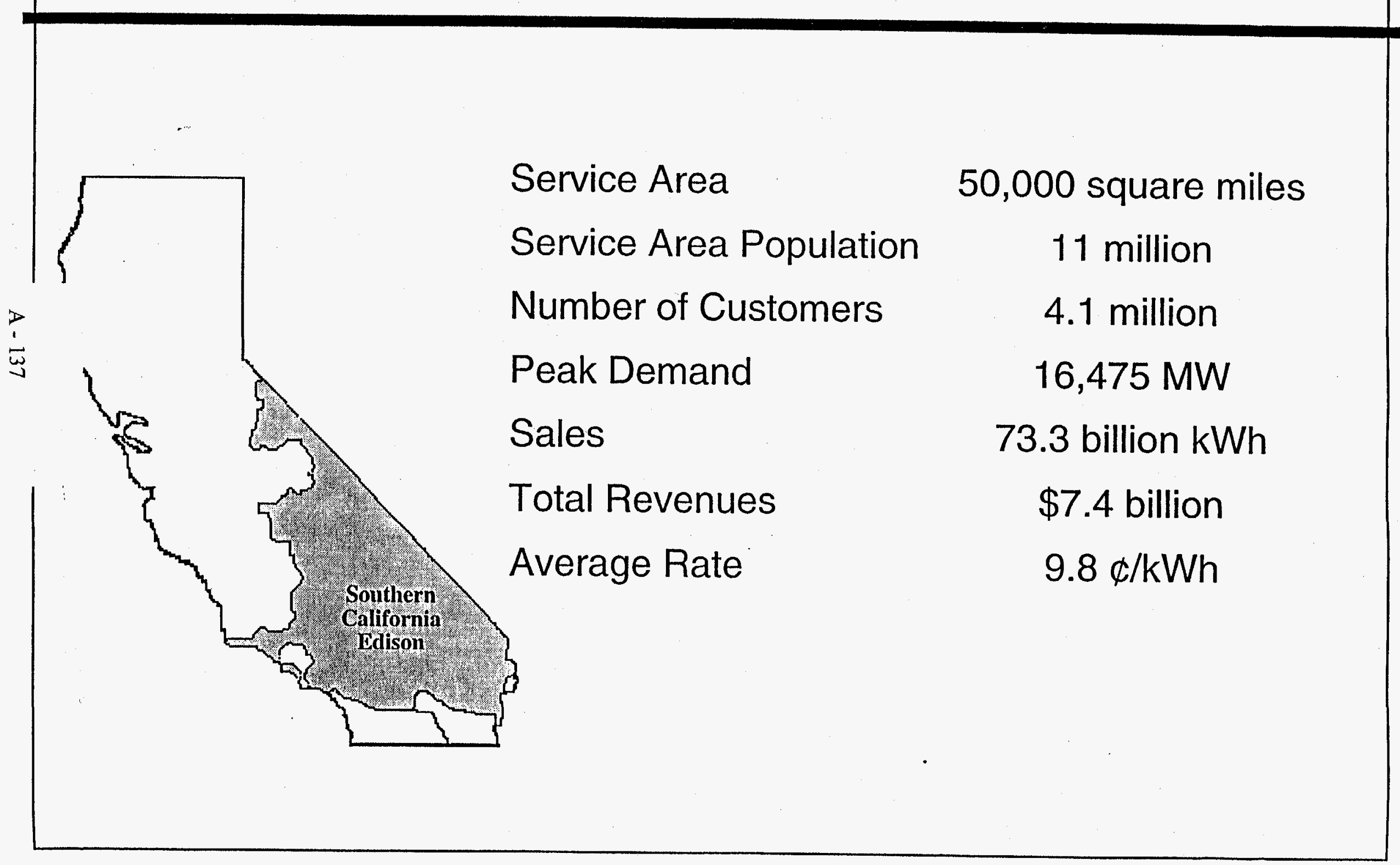




\section{Southern California Edison - 1993 Capacity and Energy Mix}

Capacity - 21,000 MW

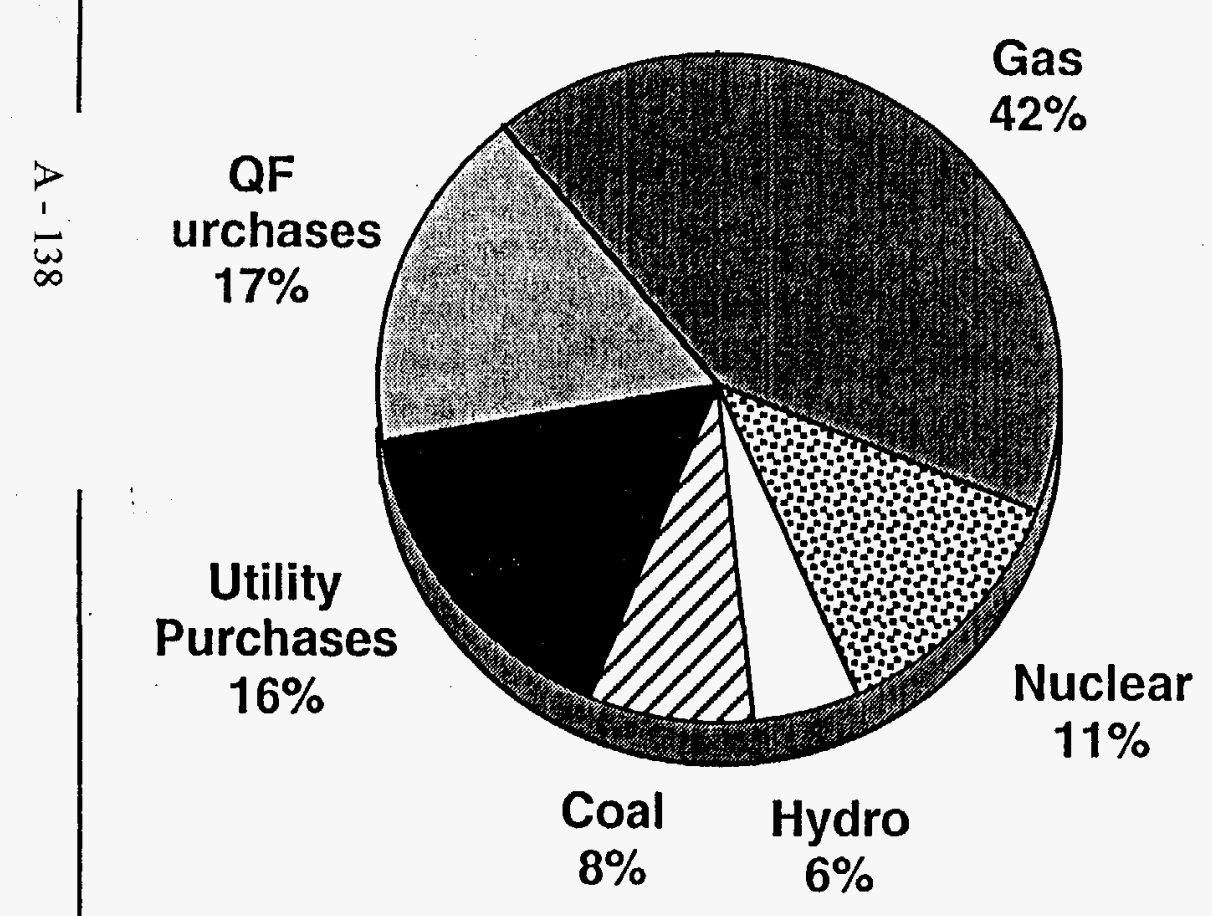

\section{Energy - $81 \mathrm{BkWh}$}

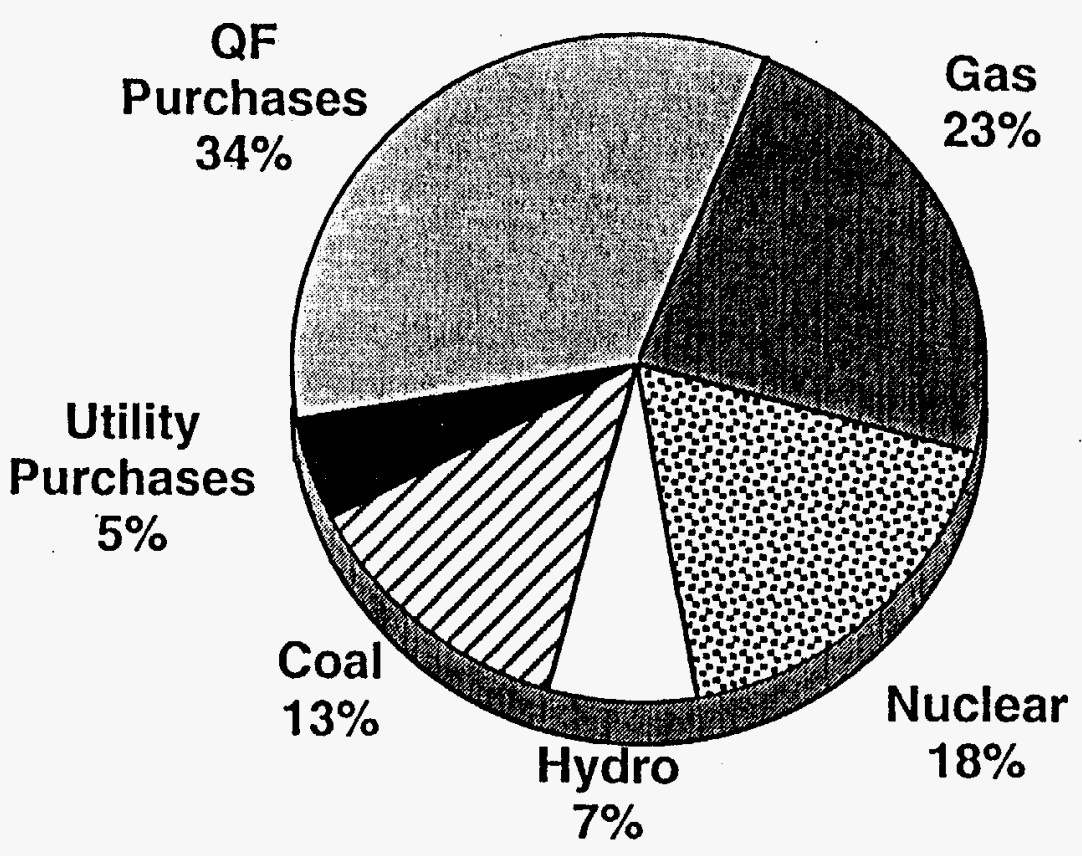




\section{Southern California Edison - Integrated Resource Plan}

(1993-2000)

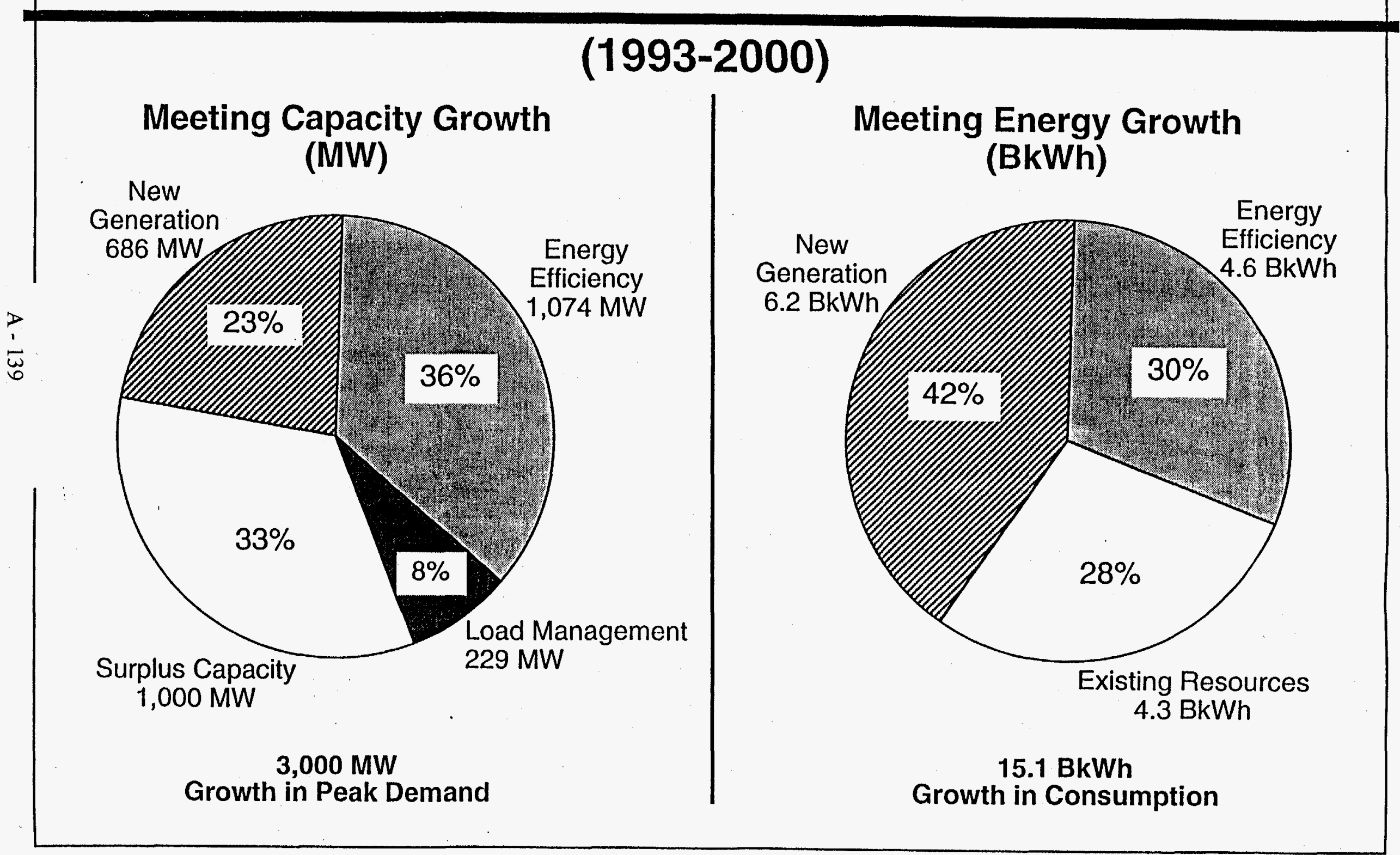




\section{State Legislation Mandates Valuation of Environmental Benefits}

- State law passed in 1990 requires valuation of environmental benefits by

- California Energy Commission ("CEC")

- California Public Utilities Commission ("CPUC")

- Legislative mandate:

"In calculating the cost effectiveness of energy resources, including conservation and load management options, the Commission shall include a value for any costs and benefits to the environment, including air quality."

- In 1992, amendments to 1990 law placed restriction on CPUC's use of environmental externalities 


\section{Resource Need Incorporates Valuation of Residual Air Emissions}

- Reliability Need

- Maintain 16\% Reserve Margin

- Loss of Load Probability $=1$ hour in 20 years

- Economic Need

- Compare Capital Investment with Fuel Savings

- Social-Economic Need

- Value Residual Air Emissions

- Value Fuel Diversity

- Integrated Resource Planning

- Demand-side resources (load management, energy efficiency)

- Supply-side resources (competitive procurement) 


\title{
Control Cost Values Initially Adopted
}

\author{
(1989 \$/Ton)
}

NOx ROG $\underline{P M-10}$ SOx $\underline{\mathrm{CO} 2}$

$\begin{array}{llllll}\text { CPUC BRPU } & * 26,400 & 18,900 & 5,700 & 19,700 & 8\end{array}$

$\begin{array}{llllll}\text { CEC ER } 90 & 18,300 & 5,200 & 12,300 & 18,100 & 8\end{array}$

* Initially proposed by South Coast Air Quality Management District for use for South Coast Air Basin; adopted by CPUC (D. 91-06-022) for use by Edison. 


\section{CPUC Applied Control Cost Values to In-State and Out-of-State Resources}

CPUC Adopted Values for Selected Technologies

(1991 $\mathrm{c} / \mathrm{kWh})$

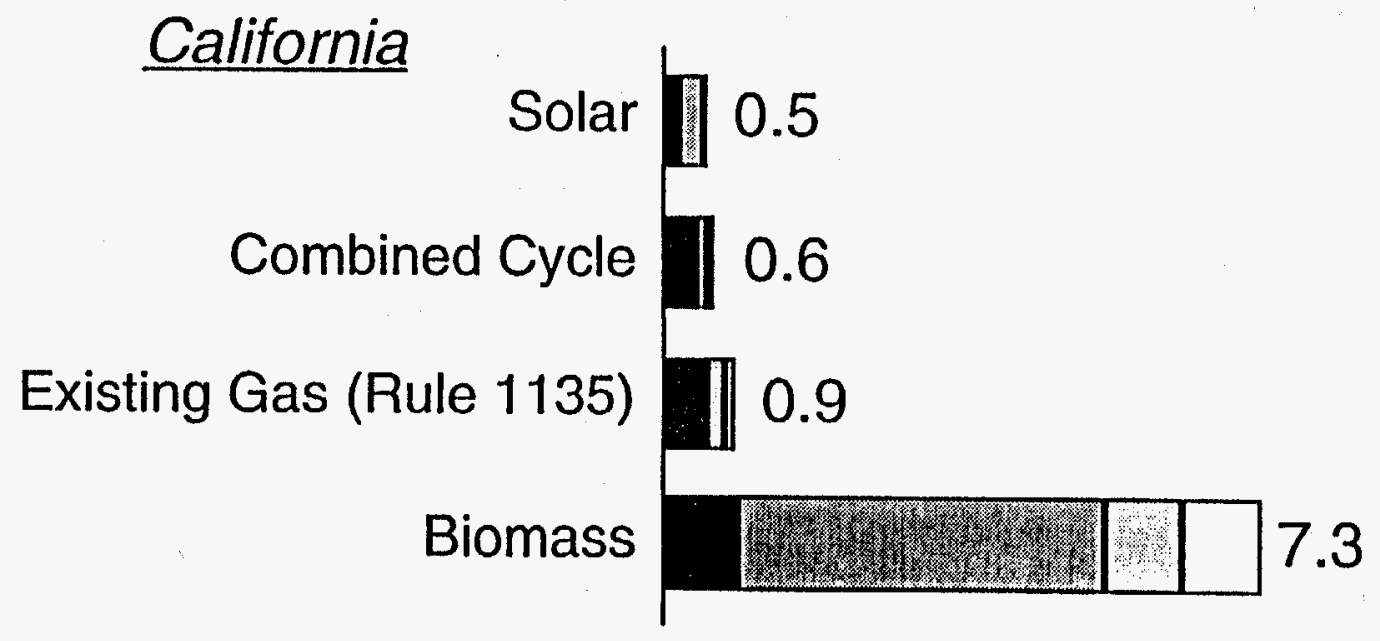

Out-of-State

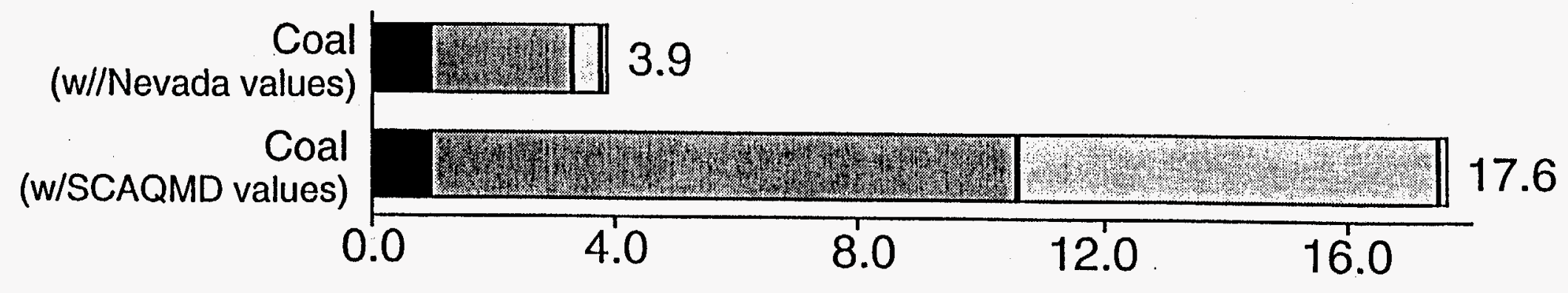




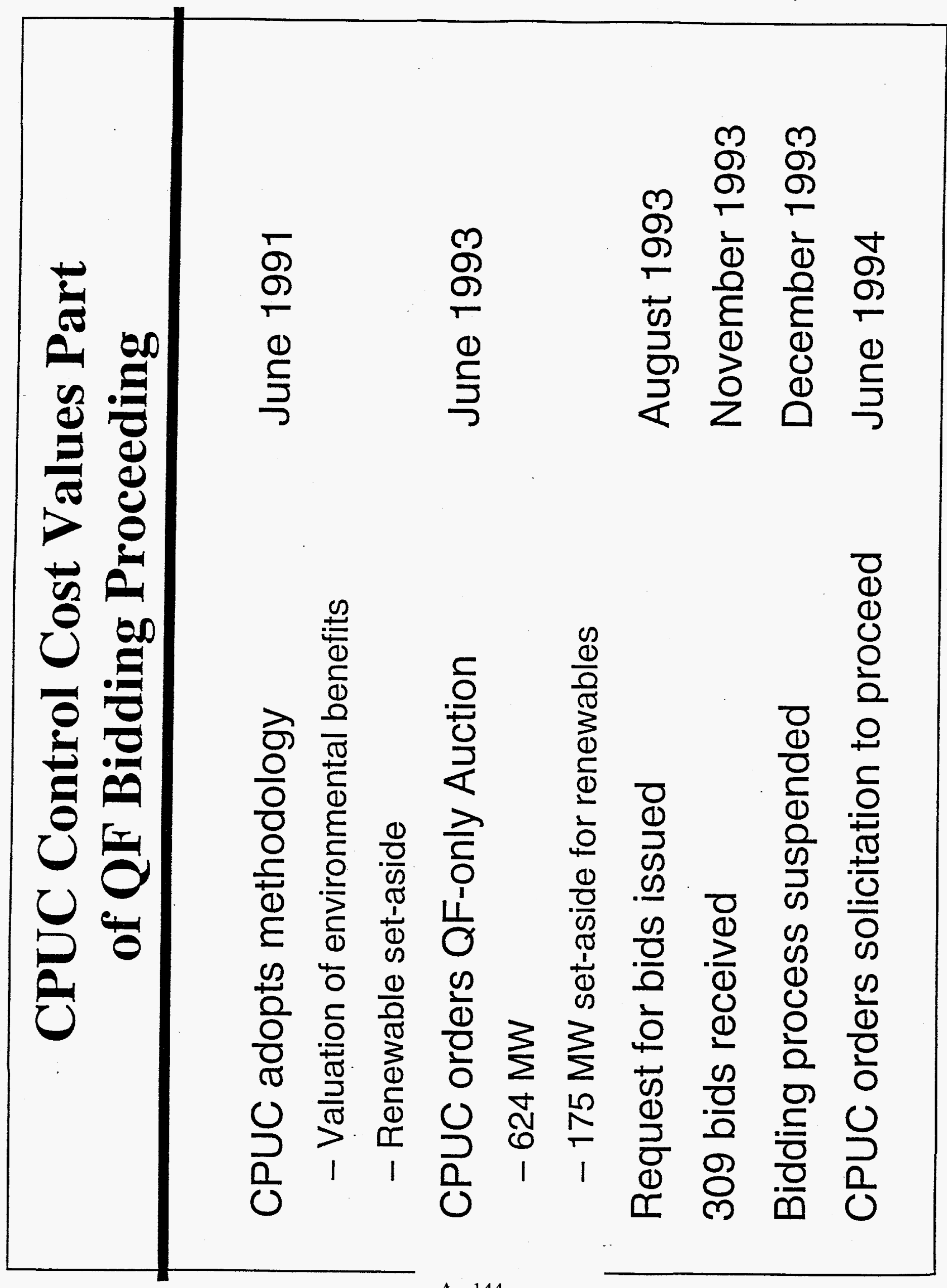




\section{Damage-Based Approach Provides Foundation for Sound Public Policy}

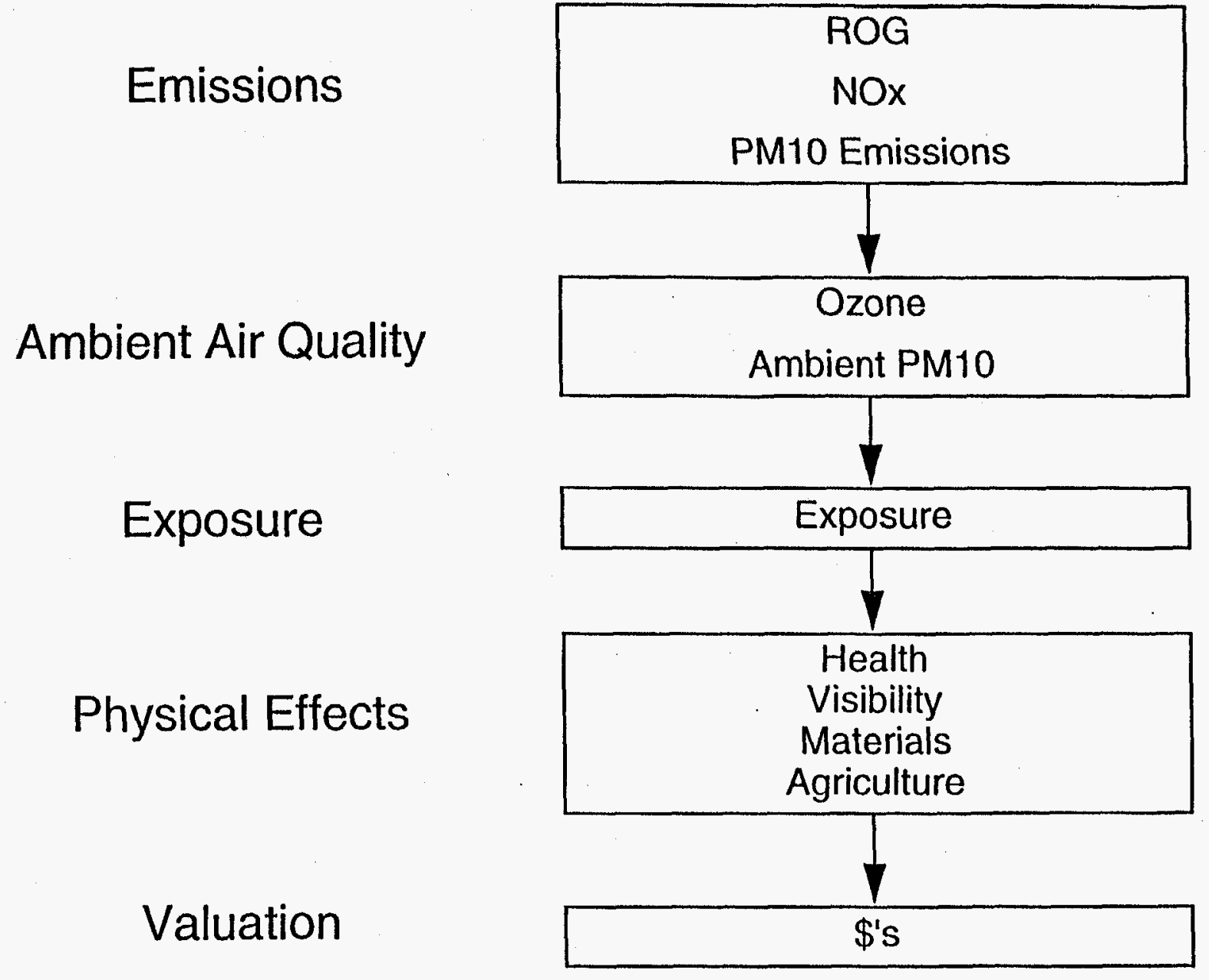




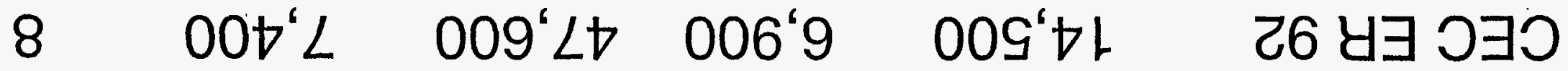 \\ $\begin{array}{lllll}0 & 009 \quad 00{ }^{\prime} \nabla t & 000 ` \varepsilon & 00 Z^{\prime} \subseteq\end{array}$ \\ *Uos!p $\exists \stackrel{0}{\frac{0}{4}}$

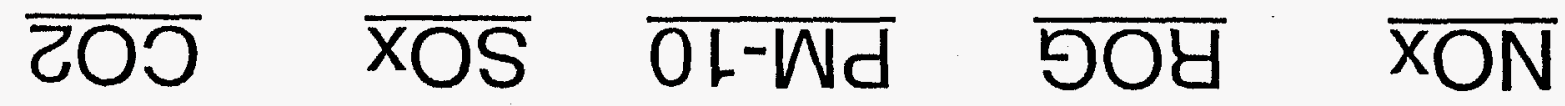

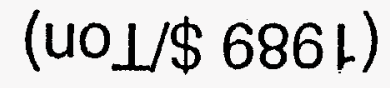

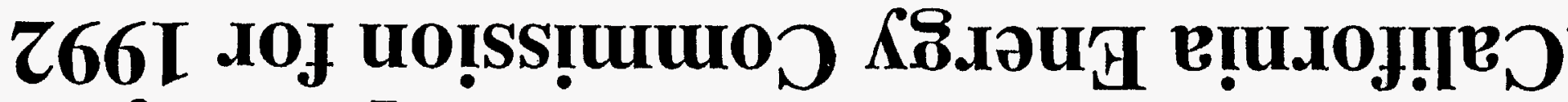

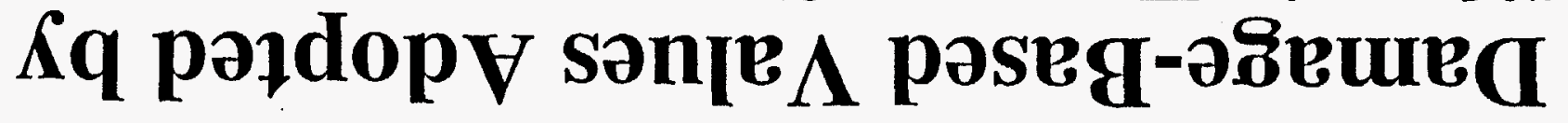




\section{Work Continues to Improve California's Damage-Based Methodology}

- Assumptions updated for 1994

- Allocation of ozone damages to primary pollutants remains unresolved

- Recommendations for further model developments

- Replacement of air quality models

- Modifications of spatial averaging for estimating exposure 


\section{CPUC Has Initiated Proposal to Restructure Electric Services Industry}

- Allow customers to choose their power supplier beginning 1996 for industrial customers and 2002 for all customers

- Recover prudent investments and preserve utility financial integrity

- Performance-based ratemaking for remaining utility services

- Maintain California's environmental and resource diversity leadership

- Lower customer rates 


\section{Restructuring Proposal May Compromise Achievement of Environmental Objectives}

Policy makers have relied on regulated utilities to further environmental and other public policy goals

- Funding of public policy programs by regulated utilities -but not other parties -- is not consistent with a highly competitive electricity market

- Development of alternative mechanisms (e.g., emission taxes, emissions trading) should be a precondition to a transition to direct access 


\section{Mechanisms for Achieving Environmental Objectives Presently Under Discussion}

- Command-and-Control Regulation

- Emission limits

- Fuel use restrictions

- Efficiency standards

- Administered Prices

- Resource planning

- Resource acquisition/payments

- Environmental Incentives

- Emissions benchmark

- Market-Based Approaches

- Emission taxes

- Marketable permits 


\section{Sound Mechanisms Recommended for Achieving Environmental Objectives}

- Broad-based mechanisms that do not unfairly disadvantage competitors

- Use of market incentives where feasible

- Valuation based on estimated damages

- Recognition of uncertainties associated with damages

- Incorporation of benefits and costs associated with externalities which are difficult to quantify 


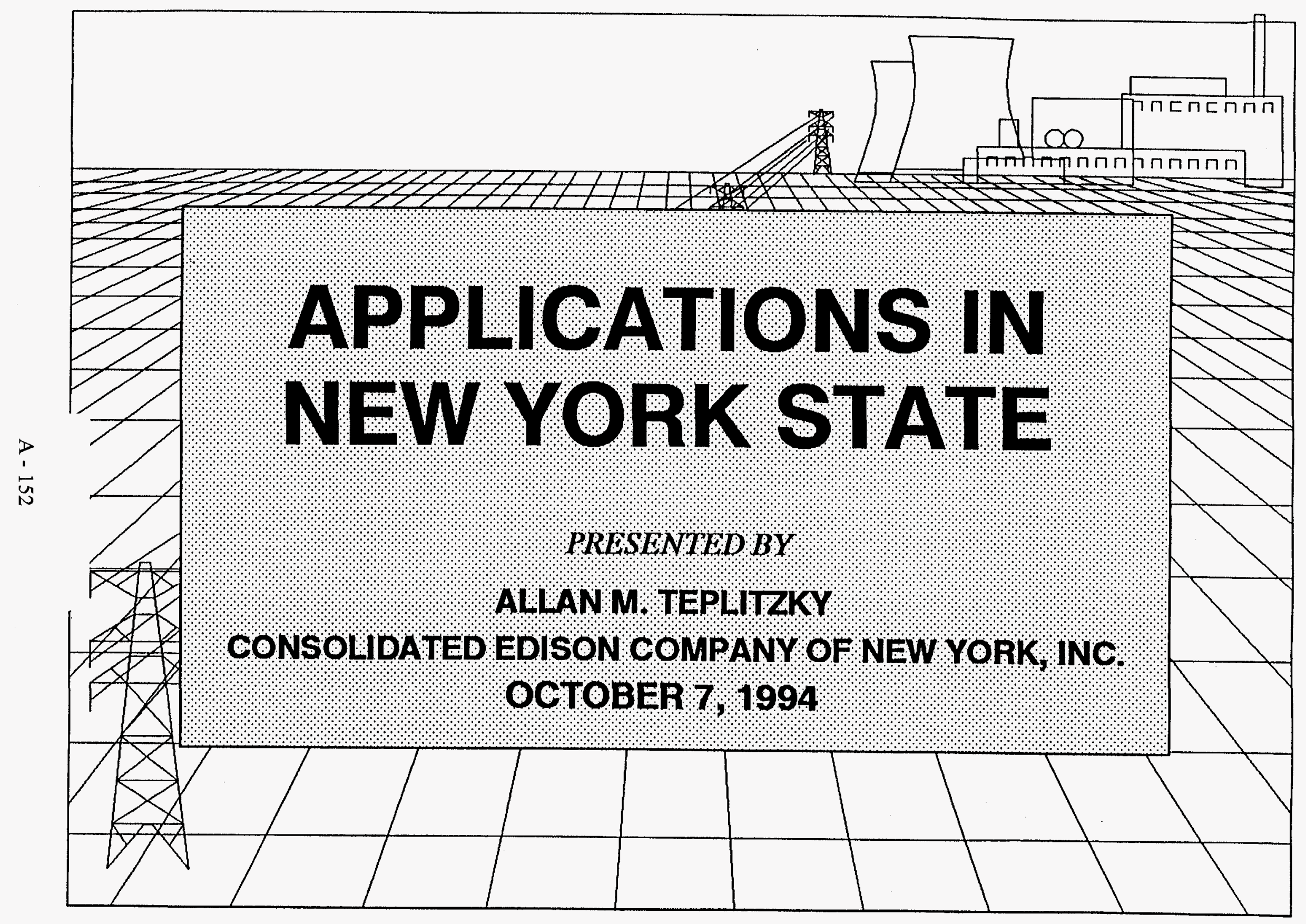




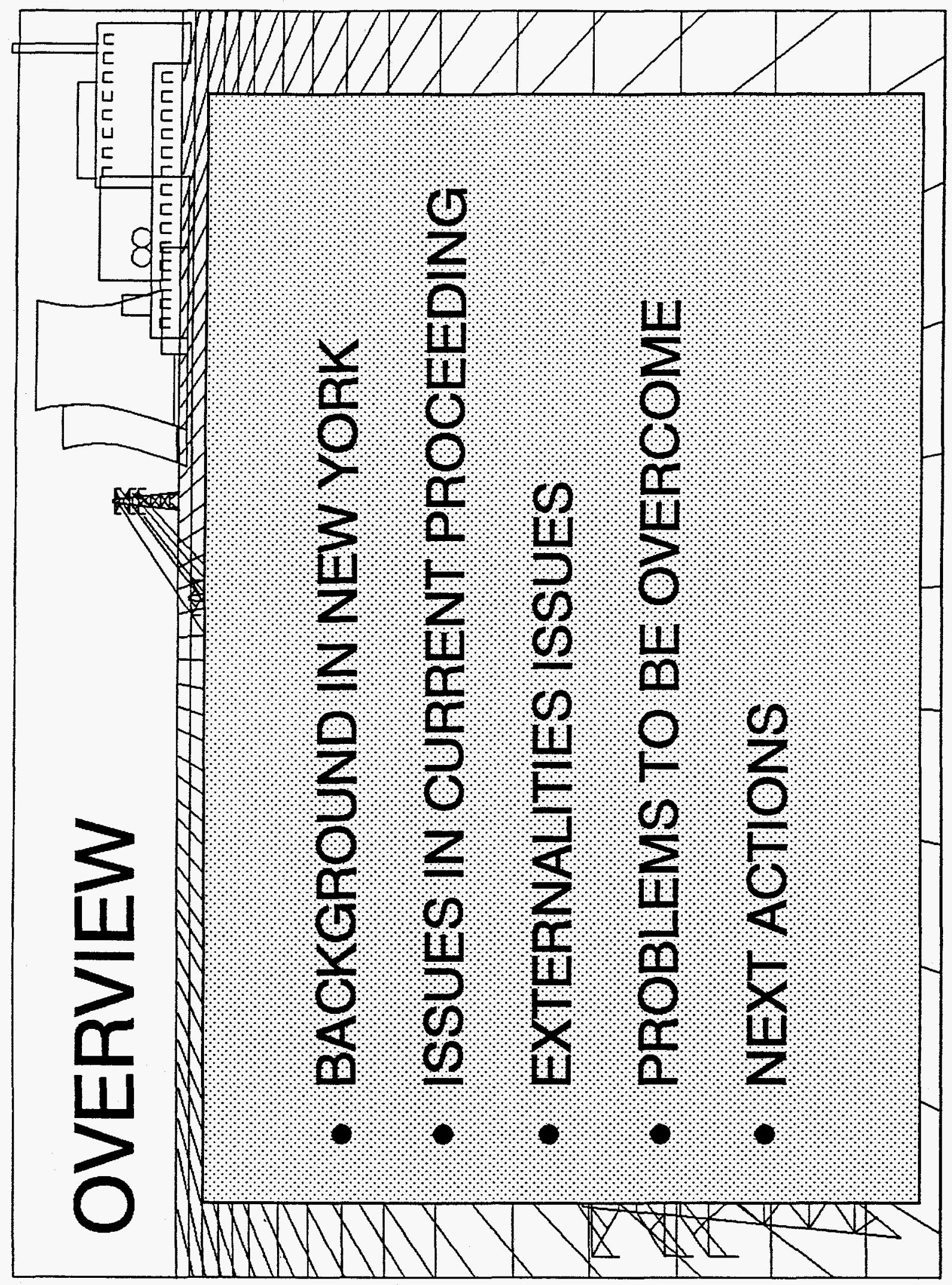




\section{BACKGROUND

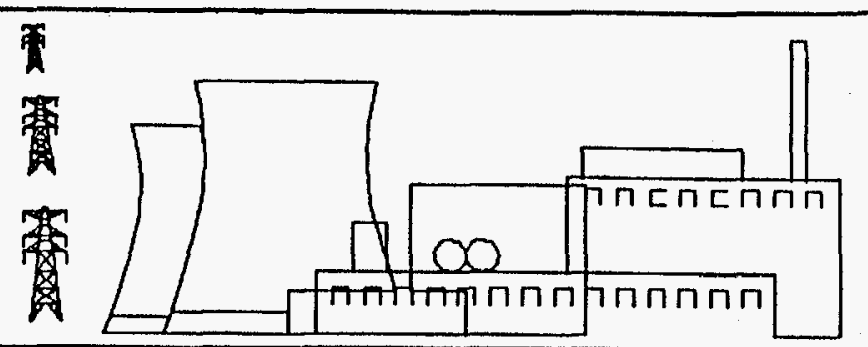

1988 - PSC ORDERS EXTERNALITIES FOR BIDDING 1989 - PSC ORDERS UTILITIES TO FUND EXTERNALITY STUDY 1991 - ALJ RECOMMENDS SEPARATE EXTERNALITIES CASE 1991 - EXTERNALITY COST STUDY INITIATED 1992 - SEP INCLUDES EXTERNALITY VALUES 1992 - PSC INITIATE PROCEEDING ON EXTERNALITY ISSUES 1994 - DRAFT SEP INCLUDES EXTERNALITY VALUES 1994 - AWAITING ALJ DECISION ON POLICY ISSUES 1994 - EXTERNALITY STUDY TO BE COMPLETED 


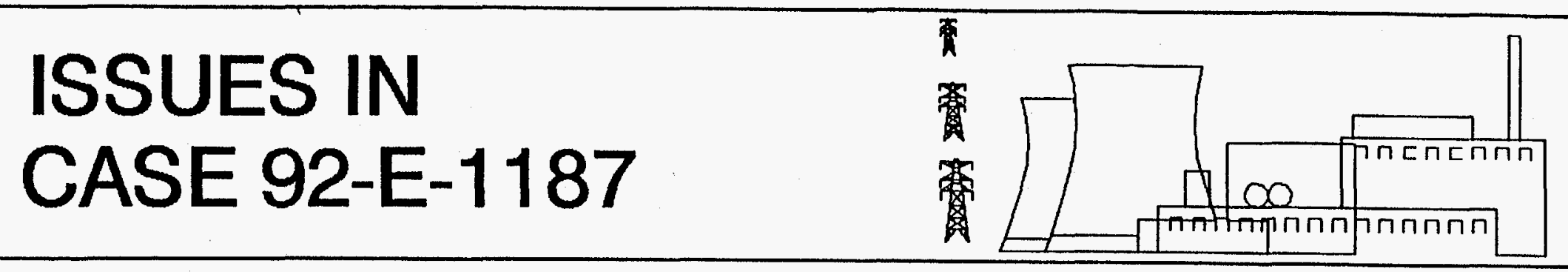

SEVENTEEN ISSUES ARE BEING CONSIDERED IN FIVE TRACKS

TRACK I - CAAA COMPLIANCE ADJUSTMENTS

TRACK II - POLICY

TRACK III - METHODOLOGICAL ISSUES

TRACK IV - VALUATION ISSUES

TRACK V - TOTAL COST DISPATCH 


\section{ISSUES RELATED USING "ADDERS"}

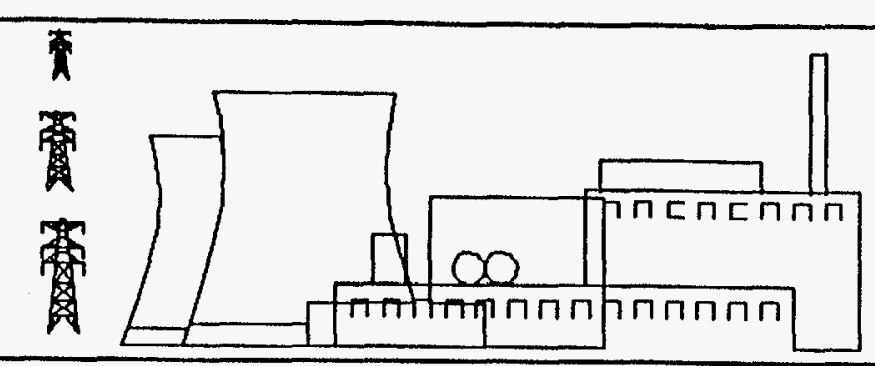

- UNLIKELY TO IMPROVE ENVIRONMENT

- DAMAGE A WEAK NEW YORK ECONOMY

- CONFLICT WITH INCREASING COMPETITION 


\section{COMPETITION ISSUES}

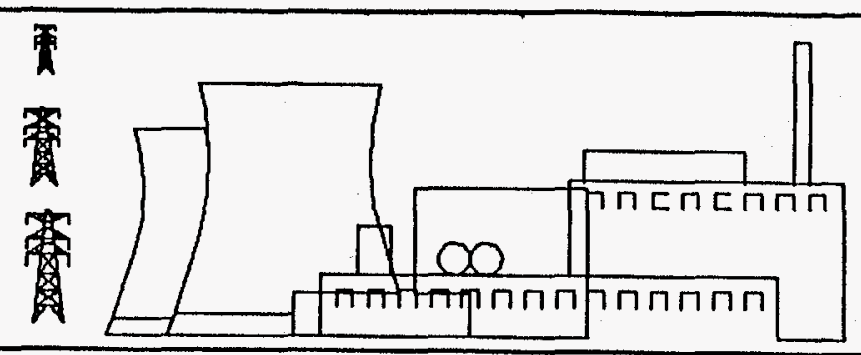

- MECHANISTIC PROCEDURES

- BYPASS

- PIECEMEAL PROBLEM

- ELECTROTECHNOLOGIES 


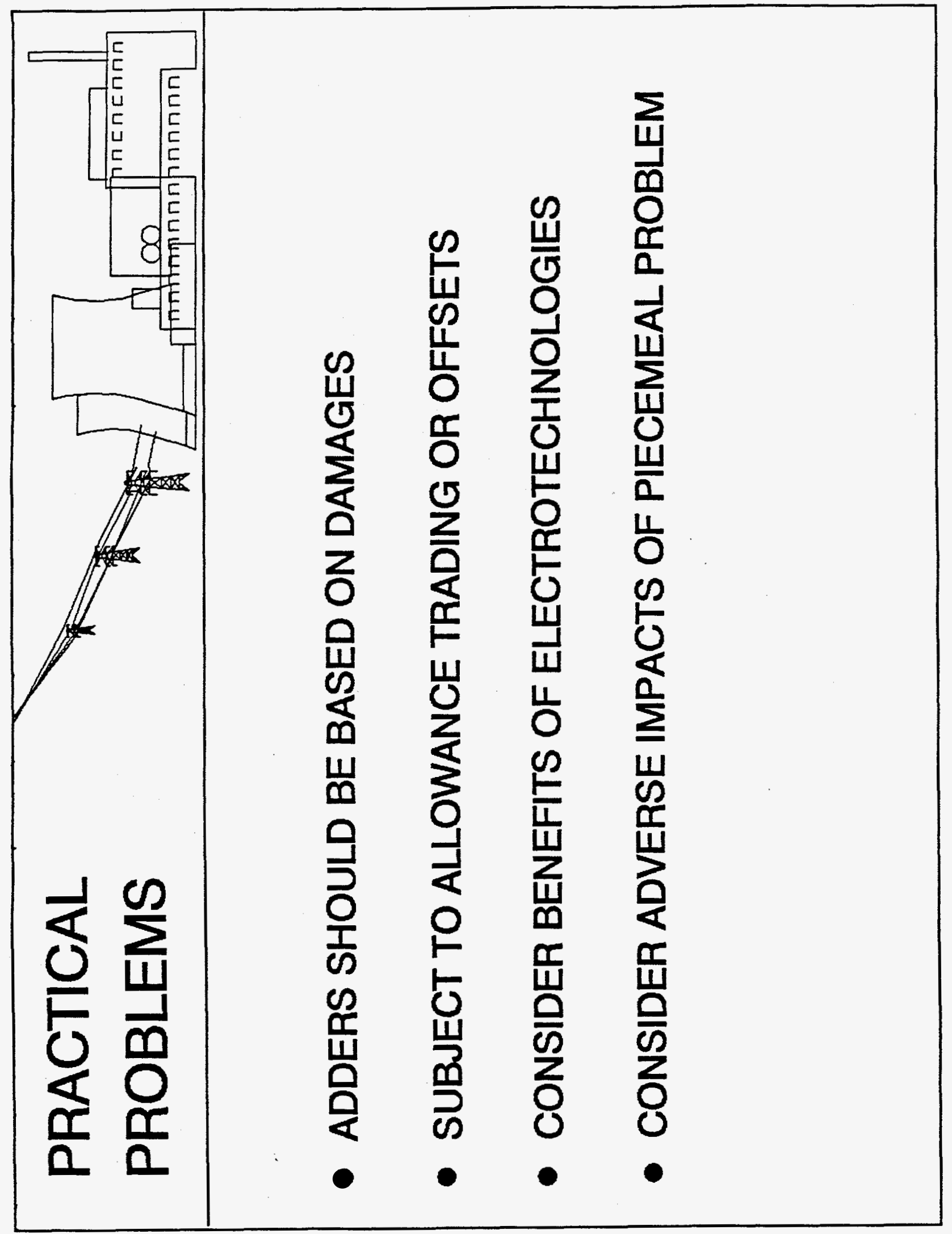




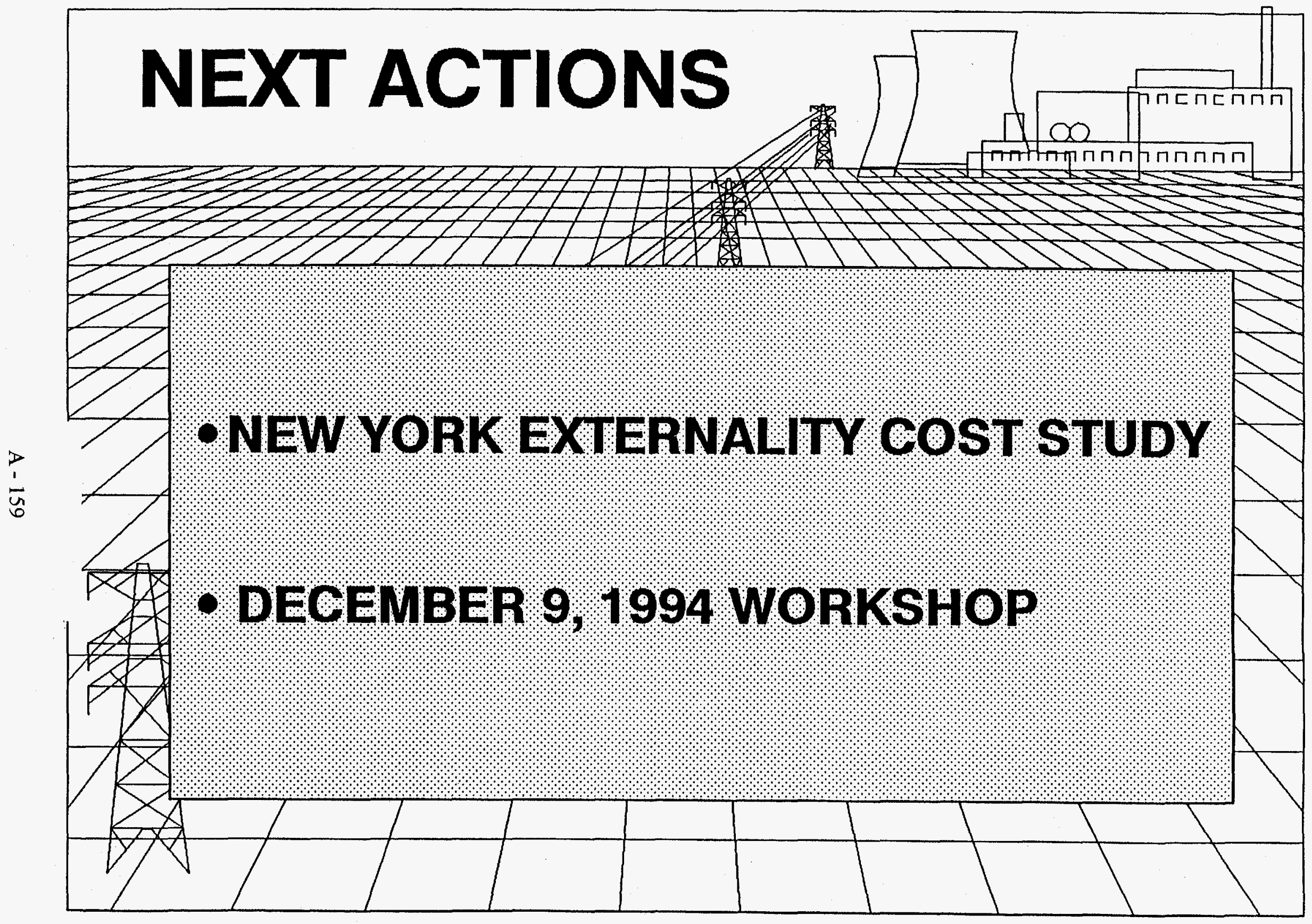




\author{
APPLICATIONS IN NEW YORK STATE \\ presented by \\ Allan M. Teplitzky \\ CONSOLIDATED EDISON COMPANY OF NEW YORK, INC. \\ New York, N.Y. 10003 \\ October 7, 1994
}

My name is Allan Teplitzky, and I will present my views concerning the use of externalities ${ }^{1}$ in the utility decision making process and the effect that the use of externalities could have on electric rates and on competition in the electricity market (Figure 1).

I will briefly discuss several areas that are of concern to my utility and to several of the other utilities in New York State. My presentation will cover several areas: the background of the externality issue in New York, issues in a current Public Service Commission ("PSC") proceeding on externalities, issues related to the use of externalities, problems that need to be overcome, and the next actions (Figure 2).

\title{
I. BACKGROUND
}

Externalities have been an ongoing issue in New York ever since the PSC ordered the utilities in 1988 to prepare a Draft Generic Environmental Impact Statement ("DGEIS") on externalities and to include externalities in their evaluation of bids for new resources (Order 88-15) (Figure 3). Although the utilities recommended using a "rate-weight" approach in their DGEIS, the Commission prescribed objective externality values in the Final Generic Environmental Impact Statement that the utilities were required to use. This was the origin of New York's $1.4 \mathrm{c} / \mathrm{KWH}$ externality cost for an NSPS coal fired power plant.

In 1989, the PSC ordered the utilities to undertake a study in conjunction with other agencies and with independent experts to develop a methodology for estimating externality costs for new resources (Opinion 89-15). That study was initiated at the end of 1991, and it is expected to be completed by the end of 1994 .

In a separate case concerning Long-Run Avoided Cost, the Administrative Law Judge recommended that a new proceeding on externalities should be initiated (Case 92-E-0886), and in 1992 the PSC started a proceeding concerning LRACs and externalities (Case 92-E1187). That proceeding is in progress and the parties are awaiting the ALJ's recommended decision on policy issues.

1

Before proceeding further, it would be useful to define externalities. Externalities occur when the action or conduct of an entity imposes costs on other parties, and those costs are not reflected in the prices or the costs that the entity or its customers face. 
Concurrent with those activities, New York State has issued a State Energy Plan ("SEP") in 1992 and a updated draft SEP in 1994. Those plans include recommendations for the use of externalities for energy decision making and provide recommendations for generic externality values.

The PSC's proceeding on LRACs and externalities, which has been in progress for two years, is examining several issues in depth (Figure 4). In that proceeding, seventeen different questions, which have been divided into five tracks, will review virtually all aspects of the externality issue. That proceeding is expected to continue for another two years.

\section{SUMMARY OF POSITION}

As I see it, the major issue in the externality debate is whether "adders" should be used by regulated electric utilities for the purpose of reflecting environmental or other external costs in resource decisions. I submit that the use of monetized adders should be considered from three perspectives: (1) environmental policy, (2) competition in the utility industry, and (3) the competitiveness of local economies (Figure 5).

\section{(1) Adders are Unlikely to Improve the Environment}

All electric resources, new or existing, must comply with stringent environmental regulations and standards that have been promulgated by the Environmental Protection Agency ("EPA"), and in the New York State by the Department of Environmental Conservation ("DEC"). Thus, compliance by utilities with the regulations internalize environmental externalities to a very large degree. The assumption that existing utility resources are "dirty", which seems to underlie the position for using externality costs, is simply wrong.

Utilities will further internalize environmental costs as they comply with the Clean Air Act Amendments of 1990 ("CAAA"). Thus, many of the most cost-effective reductions in emissions already have been or will be achieved. Therefore, potential environment benefits resulting from the use of adders are uncertain at best.

The costs that utilities incur to comply with environmental emissions or discharge regulations, or to minimize land impacts are private costs to the utilities and their customers. Environmental regulation, therefore, causes a utility to "internalize" externalities. Nevertheless, there may be residual emissions that may impose damages on society even when the utilities fully comply with environmental regulations. To the extent that these 
damages are not internalized, some externalities may remain ${ }^{3}$. However, as environmental standards become more and more stringent, any residual emissions become smaller, as do any potential damages. Therefore, the externalities become less and less significant.

\section{(2) Adders Will Damage a Weak Economy}

From an economic perspective, any increase in the prices that utilities must pay for new resources as a result of using externalities in resource decisions will increase charges to utility customers. Higher rates would have a disproportionate impact on low income consumers. Higher utilities rates may have serious adverse economic consequences for a state's overall economic competitiveness as well as its ability to attract new businesses or to retain existing businesses. For example, New York State has among the highest electric rates in the country and a continuing job loss at a time when the economies of other states are improving. From August 1989 through August 1993, the State lost almost 550,000 jobs ${ }^{4}$ and by the end of 1992 New York's share of U.S. output fell to its lowest level ever recorded ${ }^{5}$.

\section{(3) Conflict With Competition}

From a policy perspective, adding externalities to electric utility costs, but not adding them to Non-Utility Generators ("NUGs") or to other competitors costs, would give nonmarket based competitive advantage to the NUGs. It would set off a spiral of higher utility rates, which will be paid principally by those least able to choose other alternatives.

Environmental adders raise the price of electricity provided by regulated utilities, but not the prices of alternative suppliers of electricity and other forms of energy. Indeed, if one use of monetized externalities would be their inclusion in payments to non-utility generators ("NUGs"), rather than just paying the NUGs the contracted or bid price for electricity, the result would be a direct subsidy to that group. Consequently, adders create

3

The existence of residual emissions does not necessarily imply that damages are not internalized. For example, the damages that remain under an allowance trading program may be fully internalized.

Labor statistics issued by the New York State Department of Labor (September 1993).

Report of the Member Electric Systems of the New York Power Pool. Electric Planning Strategy, Vol. 1 at 18 July 1, 1994 (filed pursuant to Section 6-106 of the New York State Energy Law) (N.Y. Energy Law \& 6-106 (McKinney Supp. 1993).

In this document, the term "NUGs" refers to developers whose projects are qualifying cogeneration or small power production facilities ("QFs") under the Public Utility Regulatory Policies Act of 1978 ("PURPA") and/or eligible for the statutory minimum rate pursuant to Section 66-c of the New York Public Service Law. N.Y. Pub Serv. Law \& 66-c (McKinney 1989 and Supp. 1993) 
an artificial incentive for customers to choose alternative energy supplies, even if those supplies cause greater environmental damage or have higher social costs. As a result, the use of adders may lead to the choice of energy options with higher social costs, and may actually worsen environmental quality.

Federal and state legislation and regulatory action have significantly increased competition in the provision of electric energy. Utilities are prepared to compete, but not in shackles. There must be recognition that externality adders are inconsistent with increased competition in the electric industry.

\section{COMPETITION ISSUES}

While in theory adders could lead to regulated utilities comparing or selecting resource options on the basis of social costs ${ }^{7}$, they are more likely to increase distortions between options provided by regulated utilities and those provided by other, less highly regulated sources (Figure 6). If regulated electric utilities alone were forced to internalize environmental externalities, a perverse incentive would be created for energy customers to switch to alternative energy resources. Additionally, increased costs will deter customers from switching from other energy sources to electricity, even though electrotechnologies may offer environmental benefits. Therefore, any use of externalities by only regulated electric utilities would lead to the selection of an inappropriate resource mix. Moreover, those other sources, which are not required to internalize externalities, may well have a greater total social cost (private cost plus external costs) than the utility source ${ }^{8}$.

Competition issues concern the regulated utilities use of mechanistic procedures for including externalities in decision making, the "bypass" and "piecemeal" problems, and the discouragement of electrotechnologies by increased electric costs caused by the use of externalities.

\section{(1) Mechanistic Procedures}

From a planning perspective, I believe that the present system for evaluating additional resources provides the appropriate framework for considering all factors necessary for utility decisions to acquire future resources, including appropriate environmental factors. In a competitive environment, utilities must be permitted to make pragmatic judgments about the trade-offs among environmental, other technical, and financial concerns.

Social costs are the "sum of the private costs borne by utilities (and other firms and economic actors) and external damages that are bome by other parties but are not reflected in the costs faced by utilities and their customers."

For an example, adders may make a customer choose an option such as self-generation or NUG purchase that have higher total social costs than the utility's options. 
Requiring that externality factors be considered in a rigid and mechanistic fashion when selecting new resources would adversely affect this process.

Mandatory externality adders, or in some cases subsidies, would have an adverse impact on utility rates and on the economy. Any increases in the prices that utilities must pay for alternative resources (including Demand Side Management ("DSM")) would increase the rates utility customers are charged. These increases could be costly to ratepayers, especially low income consumers. If one cent per kilowatt hour were added to the cost of electricity produced in New York, the cost of electricity to New York consumers would be increased by over $\$ 1$ billion annually?.

All proposals for the use of inflexible monetized environmental adders in the utility planning process should be rejected. Besides the competitive, economic, environmental and practical difficulties of implementing monetized adders, there are other important reasons for not adopting them.

The types of decisions for which externality adders have been proposed to be applied range from the evaluation of major resources that may be utilized for many years to the immediate decisions about which units in the system to dispatch to meet varying loads. Other types of decisions include LRAC-based QF payments to NUGs, competitive bidding and selection of new resources, evaluation of DSM programs, system dispatch, and out-ofstate purchases. Each of these decisions involves various factors, including: economic, engineering, environmental, and social. The weight placed on each of these effects varies considerably depending on the resource under review. A rigid application of a monetized adder to each of these decision would distort the selection process and diminish the value of the resulting decision.

\section{Piecemeal and Bypass Problems}

Utility customers, who have an opportunity to choose, will buy electricity from the supplier who offers service with equivalent reliability and quality at the lowest price. An environmental externality policy that increases utility rates, but has no effect on (or even subsidizes) alternative energy suppliers, will encourage customers, particularly large users of electricity, to buy from those alternative suppliers or to become a self-generator. Customers who cannot choose a different supplier will have to pay for their share of the additional charges, as well as for the share of the fixed costs that otherwise would have been paid by former customers.

Report of the Member Electric Sustems of the New York Power Pool, Load \& Capacity Data, Vol. 2 at 22. Figure I-14 (July 1, 1993) (filed pursuant to Section 6-106 of the New York State Energy Law) (N.Y. Energy Law 6-106 (McKinney Supp. 1993). The $\$ 1$ billion figure is based on 1992 energy consumption of 143,431 GWH. 
Moreover, some industrial customers will choose not just to leave a utility's system with already high rates, but they will choose to leave a geographic area. In 1992, the New York statewide average electric price was 10.30 cents per kilowatt hour and the national average was 6.80 cents per kilowatt hour ${ }^{10}$. To make the State more competitive, the 1992 New York State Energy Plan ("SEP") sets as a goal the proposal that New York reduce the gap between the energy price in New York and the national average by at least 50 percent by $2008^{11}$. Any policy, including the use of externalities, that increases New York electric prices increases the likelihood that this goal will not be met.

Thus, burdening only regulated electric utilities with externality adders creates a "piecemeal" regulatory problem. Adders reflecting externality costs should not be applied solely to regulated electric utilities.

\section{(3) Environmental Benefits of Electrification}

Externality proponents dwell on damages produced by residual emissions from utilityowned electric generation facilities. However, any external costs from electric generation must be balanced against the social benefits, including environmental benefits, that electricity provides. The effect of electricity on the environment must take into account emissions at the point of use as well as emissions at the point of generation.

"Electrotechnology" is a broad term for a variety of processes that allow manufacturers to improve quality and to increase competitiveness and productivity by the use of electricity. Electrotechnologies allow precise control and focus of energy only when and where it is needed, and it can be more efficient than using fossil fuels. Electrotechnology processes often displace more polluting energy sources. Caution should be used in considering a policy in which electric rates increase due to the inclusion of monetized externality adders, because such a policy not only ignores, but actively discourages the associated economic and environmental benefits arising from the efficient use of electricity.

\section{PRACTICAL PROBLEMS}

In the event that environmental adders are mandated in the planning process, it is recommended that consideration be given to the concerns discussed above in order to minimize the impacts on the electric utility industry and its ratepayers (Figure 7). At a minimum:
10
U.S. Department of Energy/ELA, Monthly Electric Utility Sales and Revenue Report with State
Distributions, Form ElA-826 March 1993.
11
SEP, Vol. II, Plan Report, at 17 (February 1992). 
(1) adders should be based on reliable measures of the damages caused by emissions and not on the fundamentally flawed compliance or abatement cost methods;

(2) adders should reflect only the costs attributable to residual emissions and that emissions subject to allowance trading or offsets should be completely excluded (Appendix A);

(3) proper recognition should be given to the environmental benefits of the consumers' uses of electricity, such as electrotechnologies; and

(4) proper recognition should be given to the adverse environmental impacts and economic hardships that will occur when higher retail rates cause electric power consumers to shift to unregulated electric suppliers that are not required to consider social costs when acquiring their generating resources.

\section{CONCLUSION}

In recognition of the complexities associated with the proper valuation of externalities and in compliance with the PSC's Opinion No. 89-15, the New York utilities through their research arm, the Empire State Electric Energy Research Corporation ("ESEERCO"), are sharing, along with State government, New York State Energy Research and Development Authority ("NYSERDA"), the Electric Power Research Institute ("EPRI") and non-utility sources, the funding and managing of a major study, "New York State Environmental Cost Study" (Figure 7). One report in the Study, "Externalities Screening and Recommendation," was released in December 1993, and the entire study is scheduled to be completed by the end of 1994. A workshop to review that study has been scheduled for December 9, 1994, in Albany, N.Y. In conclusion, any action concerning the use of environmental adders in the planning process should be deferred until the results of the New York study are available. 


\section{APPENDIX A}

\section{Environmental Regulation Internalize Environmental Damages}

As a result of the CAAA, all of the external costs which theoretically could be associated with emissions of sulfur dioxide (" $\mathrm{SO}_{2}$ ") and nitrogen oxides $\left(" \mathrm{NO}_{x}{ }^{\prime \prime}\right)$ have been or shortly will be internalized at facilities because of the requirement that owners obtain $\mathrm{SO}_{2}$ allowances or $\mathrm{NO}_{x}$ offsets.

National ambient air quality standards ("NAAQS") are intended to protect public health with an adequate margin of safety, and to protect the public welfare from any known or anticipated adverse effects associated with air pollutants. In New York, the NAAQS for $\mathrm{SO}_{2}$ are being met; thus, the health and welfare of this State's residents are adequately protected insofar as ambient $\mathrm{SO}_{2}$ levels are concerned. The costs of achieving the ambient $\mathrm{SO}_{2}$ standards have already been internalized by the utilities.

In addition, to minimizing pollutants in the ambient air, utilities have been and are acting to minimize acidic deposition ${ }^{12}$. Title IV requires all electric utilities in the continental United States to reduce sulfur dioxide emissions from electric utility generating plants to less than 8.9 million tons annually by the year 2000 , at a cost of billions of dollars to electric customers. Those costs are now being internalized, using free market mechanisms to do so efficiently.

The recent introduction of national $\mathrm{SO}_{2}$ allowance trading, and the evolving plans for trading in $\mathrm{NO}_{\mathbf{x}}$ (and possibly other pollutants), internalize the costs of residual emissions. Emissions trading programs create a market for emission allowances or credits. Economists consider emissions trading to be an appropriate means for internalizing an externality.

$\mathrm{NO}_{x}$ from all emission sources, including fossil fuel-fired generators (whether utility or NUG) in combination with volatile organic compounds ("VOCs"), are precursors atmospheric processes that produce ozone $\left(\mathrm{O}_{3}\right)$ in the ambient air. Additional controls on $\mathrm{NO}_{\mathrm{x}}$ emissions will soon be required under the ozone non-attainment provisions of the CAAA. As the next step toward attainment of the ambient air quality standard for ozone in New York, the DEC has promulgated reasonably available control technology ("RACT") limits for $\mathrm{NO}_{\mathrm{x}}$ emissions from electric generating units and other major stationary sources $^{13}$. These standards must be implemented by May 31, 1995.

NAAQS limit the concentration of pollutants, such as $\mathrm{SO}_{2}, \mathrm{NO} \mathrm{x}$ and particulates, in the ambient air used by people, animals and plants for respiration. Acid rain programs act to minimize the deposition of aerosols from derivatives of $\mathrm{SO}_{x}$ and $\mathrm{NO} x$ on land and water, because those derivatives (i.e., $\mathrm{SO}_{3}$, $\mathrm{SO}_{4}$, and $\mathrm{NO}_{3}$, can acidify unbuffered water bodies and land areas.

See proposed 6 NYCRR Part 227-2, Reasonably Available Control Technology for Oxides of Nitrogen. 
$\mathrm{NO}_{\mathrm{x}}$ offset requirements further internalize costs and, in fact, provide benefits in many areas greater than are required. In short, the costs of ozone attainment have been or shortly will be fully internalized through new controls, $\mathrm{NO}_{x}$ trading, and offsets.

\section{Global Warming}

A major externality issue continues to be the correct methodology for establishing an externality value for the emissions of carbon dioxide $\left(" \mathrm{CO}_{2}{ }^{\prime \prime}\right)$ and other greenhouse gases. Carbon dioxide is a global issue and should be addressed at national or international levels ${ }^{14}$. Carbon dioxide emissions from electric power plants in New York constitute a very small percentage of annual man-made global $\mathrm{CO}_{2}$ emissions. One estimate if approximately 0.3 percent ${ }^{15}$.

Utilities should not alone bear the costs of providing a benefit to the rest of the nation or this world. The country would be better served if the EPA and Congress would develop a national $\mathrm{CO}_{2}$ strategy so that all sections of society would be on a level playing field in terms of the economic impact of $\mathrm{CO}_{2}$ controls on all forms of energy use.

It should be noted that there remains considerable controversy in the scientific community as to the ultimate effects of $\mathrm{CO}_{2}$ emissions. Stevens, Scientist Confront Renewed Backlash on Giobal Warming. N.Y. Times, at C1, Sept. 14, 1993. tion Analysis Center, Oak Ridge (199)). In 1990, New York State electric utilities' share of $\mathrm{CO}_{2}$ emissions were approximately 64 million tons. New York State Energy Office, Analysis of Carbon Reduction in New York State, at 32 (June 1991). 
Total Fuel Cycle Assessment Workshop

"Gas Utility Applications"

\author{
Austin, Texas \\ October $6-7,1994$ \\ Wilson Gonzalez \\ Coordinator, IRP
}

Columbia Gas Distribution Companies

\title{
ABSTRACT
}

In the development of its initial Gas IRP, Columbia Gas of Maryland, Inc. (CMD) prepared a Total Fuel Cycle analysis (TFCA) of the various gas and alternative fuel equipment available to its customers. The TFCA supported and supplemented the positive economics of gas equipment depicted in the standard DSM costbenefit analysis contained in CMD's IRP filing. The purpose of the TFCA analysis was to expand the level of information and data useful in IRP related decisions made by regulators. : The IRP movement is concerned with economic efficiency, energy efficiency and reducing the degradation to the environment (and other social benefits). Unfortunately, the current IRP process as practiced in many states looks at electric IRP in isolation. Electric utilities are encouraged to intervene in the energy marketplace and offer financial incentives for equipment, that while more efficient than standard electric equipment, can be less efficient from a resource basis than standard gas equipment. This policy's impact is that customers are favoring less resource energy efficient electric technologies (because of the electric utility incentives) than the more efficient gas equipment available. The IRP convention therefore, is neither integrated nor resource based. Columbia believes that, for the IRP process to be truly integrated and truly resource based, it must take into account the efficiency with which energy resources (coal, uranium, crude oil, natural gas, etc.) are produced and subsequently consumed by the various customer end uses in our economy. It must also take into account the relative environmental consequences of the production and consumption of those resource energy forms for those end uses. In this context, Columbia believes that TFCA is a means to an end (more efficient and cleaner energy use) and helps Columbia underlie the fact that the selective increased use of natural gas for a variety of consumer end uses, developed within an IRP framework, can conserve resource energy, reduce the emissions of pollutants, defer the need for construction of additional electric generation capacity, reduce the equipment to comply with the Clean Air Act Amendments, and assist for specific pollutants stipulated in the Clean Air Act Amendments. 

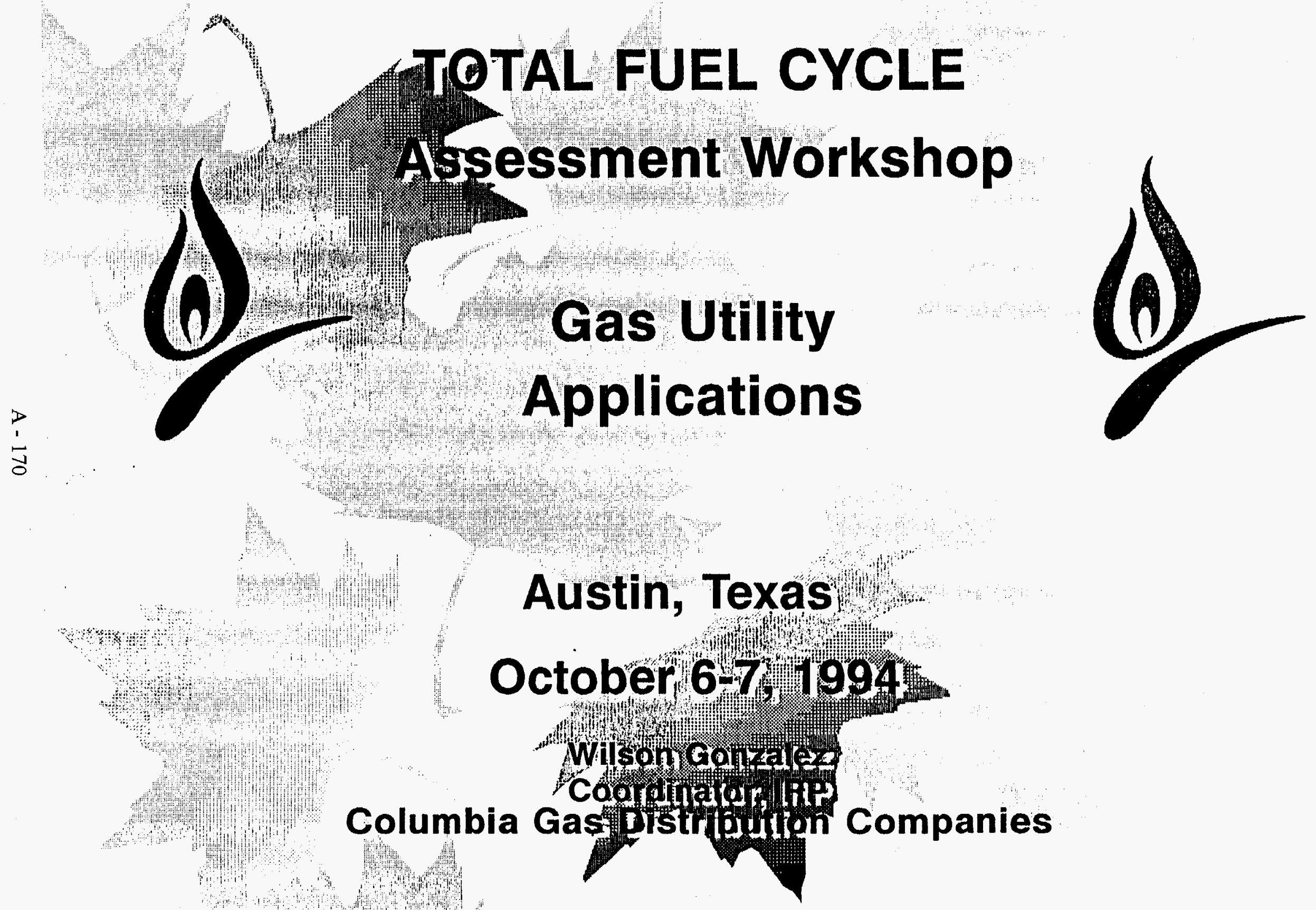


\section{Columbia Gas Distribution Companies}

-1.8 Million Customers in 5 States

- Ohio

- Pennsylvania

- Virginia

- Kentucky

- Marylr 


\section{IRP Status: Maryland}

- Legislation (7/91) requiring development and implementation of conservation and efficiency programs

- PSC directive to file IRP/DSM:

- Development Plan by 11/18/91

- Filed and Received PSC approval for two residential pilot programs $7 / 92$

- Final Plan by 10/92 (modified to Status Report)

- Final Plan by $9 / 93$ 


\section{Gas DSM Programs: How They Stack Up In CMD Residential}

\begin{tabular}{|lll|}
\hline Use/Equipment & Gas To Gas & $\begin{array}{l}\text { Electric To } \\
\text { Gas }\end{array}$ \\
\hline Space Heating & + & ++ \\
Water Heating & $-1+$ & ++ \\
Laundry Drying & - & + \\
Cooking & - & + \\
Gas Heat Pump & ++ & + \\
New Construction & + & $\mathrm{NA}$ \\
Low Income Weatherization & ++ & $\mathrm{NA}$ \\
House Doctor & $-/+$ & $\mathrm{NA}$ \\
Natural Gas Vehicle & $\mathrm{NA}$ & ++ (gasoline) \\
Combo Space \& Water Heater & - & ++ \\
\hline
\end{tabular}

(++ represents a greater than 1.75 TRC Result, + is greater than 1.0 TRC) 


\section{Gas DSM Programs: How They Stack Up In CMD}

Commercial/Industrial

\begin{tabular}{|lll|}
\hline Use/Equipment & Gas To Gas & $\begin{array}{l}\text { Electric To } \\
\text { Gas }\end{array}$ \\
\hline Space Heating & ++ & ++ \\
Water Heating & - & ++ \\
Laundry Drying & + & + \\
Food Service & $-/+$ & $-/+1++$ \\
Cooling & $\mathrm{NA}$ & $-/+^{\star}$ \\
Engine Driven Applications & $\mathrm{NA}$ & + \\
Natural Gas Vehicle & $\mathrm{NA}$ & ++ (gasoline) \\
Industrial & $-/+$ & ++ (oil) \\
\hline
\end{tabular}




\section{Treatment of Environmental Externalities}

- From a Societal Perspective, the inclusion of externalities is appropriate.

- CMD included externalities for illustrative purposes

- CMD used conservative numbers from LBL Study

- SO2 at $0.42 \$ / \mathrm{lb}$ of Emission (\$9.15 High inside SCAQMD)

- NOX at $0.92 \$ / \mathrm{lb}$ of Emission $(\$ 12.25$ "

- C02 at $0.0005 \$ / \mathrm{lb}$ of Carbon (\$0.027 " " " )

- Made a Difference in Only One Program

- Represented $\$ 62$ million dollars or 26 percent of the total aggregate program lifetime savings 


\section{Fuill Trajectory Resource Energy Efficiency}

- Natural gasiequipment is more efficient than electric equipment for selected end use applications including:

- R\&C space heating

- R\&C water heating

- R\&C cooking

- R\&C laundry drying

- C\&I space heating -

- C\&I engine-diriven equipment 


\section{Energy Trajectory Efficiencies Source to Site}

\begin{tabular}{|c|c|c|c|c|c|c|}
\hline & Extraction & Processing & Transportation & Conversion & Distribution & Cumulative \\
\hline Natural Gas & $96.8 \%$ & $97.6 \%$ & $97.3 \%$ & $\star$ & $99.2 \%$ & $91 \%$ \\
\hline $\overrightarrow{3}$ & $96.8 \%$ & $90.2 \%$ & $98.4 \%$ & $\star$ & $99.8 \%$ & $86 \%$ \\
Fuel Oll & & & & & \\
Electric & $99.4 \%$ & $90.0 \%$ & $97.5 \%$ & $33.4 \%$ & $92.0 \%$ & $27 \%$ \\
Guclear & $96.8 \%$ & $90.2 \%$ & $98.4 \%$ & $32.5 \%$ & $92.0 \%$ & $26 \%$ \\
Hydro & $96.8 \%$ & $97.6 \%$ & $97.3 \%$ & $31.8 \%$ & $92.0 \%$ & $27 \%$ \\
Other & $100 \%$ & $100.0 \%$ & $100.0 \%$ & $29.1 \%$ & $92.0 \%$ & $27 \%$ \\
Weighted & $*$ & $100.0 \%$ & $100.0 \%$ & $17.3 \%$ & $92.0 \%$ & $16 \%$ \\
\hline
\end{tabular}



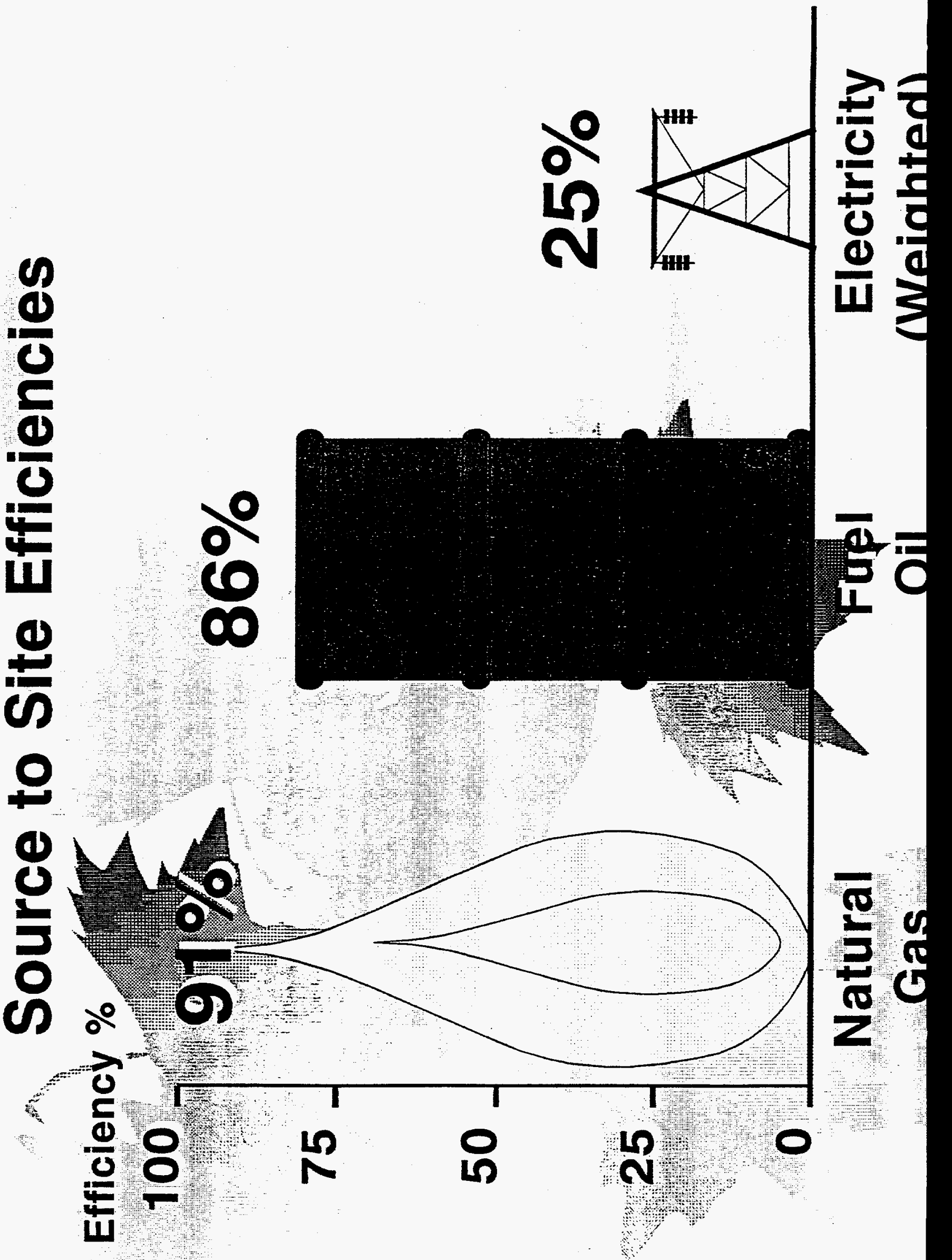


\section{Energy Trajectory Efficiency Conclusion}

- The natural gas industry delivers resource energy to the customer 3.6 times as efficiently as the electric utility industry. 


\section{Full Trajectory Resource Energy Efficiency}

Gas and Electric Space Heating Systems

\begin{tabular}{|l|c|}
\hline & \\
\hline GAS SPACE HEATING & AFUE * REE $=$ RAFUE \\
NAECA minimum GF & $0.78 * 0.91=0.71$ \\
Condensing GF & $0.94 * 0.91=0.85$ \\
& HSPF * REE $=$ RHSPF \\
Gas Engine Heat Pump & $1.40 * 0.91=1.27$ \\
& \\
ELECTRIC SPACE & HSPF * REE $=$ RHSPF \\
HEATING & $1.00 * 0.27=0.27$ \\
Resistance & $2.00 * 0.27=0.54$ \\
NAECA minimum EHP & $2.60 * 0.27=0.70$ \\
\hline
\end{tabular}




\section{Full Trajectory Resource Energy Efficiency}

Gas and Electric Water Heating Systems

\begin{tabular}{|l|l|}
\hline \multicolumn{1}{|c|}{ EF * REE $=$ REF } \\
\hline GAS WATER HEATER & \\
NAECA minimum & $0.55 * 0.91=0.50$ \\
Subchamber & $0.62 * 0.91=0.56$ \\
Subchamber - powered & $0.72 * 0.91=0.65$ \\
Subchamber - condensing & $0.85 * 0.91=0.77$ \\
& \\
ELECTRIC WATER HEATER & $0.90 * 0.27=0.24$ \\
\hline
\end{tabular}




\section{Source to Site Energy Trajectory Efficiency Ga's and Electric Space Heating Systems}

\begin{tabular}{|c|c|c|c|}
\hline & $\begin{array}{l}\text { Site } \\
\text { Efficiency } \\
\text { Rating }\end{array}$ & $\begin{array}{l}\text { Trajectory } \\
\text { Efficiency }\end{array}$ & $\begin{array}{c}\text { S/S } \\
\text { Resource } \\
\text { Efficiency }\end{array}$ \\
\hline $\begin{array}{l}\text { Gas } \\
\text { 1. NAECA minimum furnace } \\
\text { 2. Condensing furnace } \\
\text { 3. Gas Engine Heat Pump }\end{array}$ & $\begin{array}{l}\text { (AFUE/HSPF) } \\
0.78 \\
0.94 \\
1.40\end{array}$ & $\begin{array}{l}0.91 \\
0.91 \\
0.91\end{array}$ & $\begin{array}{l}0.71 \\
0.85 \\
1.27\end{array}$ \\
\hline $\begin{array}{l}\text { Electric } \\
\text { 1. Resistance furnace } \\
\text { 2. NAECA minimum } \\
\text { Heat Pump } \\
\text { 3. High Efficiency } \\
\text { Heat Pump }\end{array}$ & 2. & $\begin{array}{l}0.27 \\
0.27\end{array}$ & $\begin{array}{l}0.27 \\
0.54 \\
0.70\end{array}$ \\
\hline
\end{tabular}




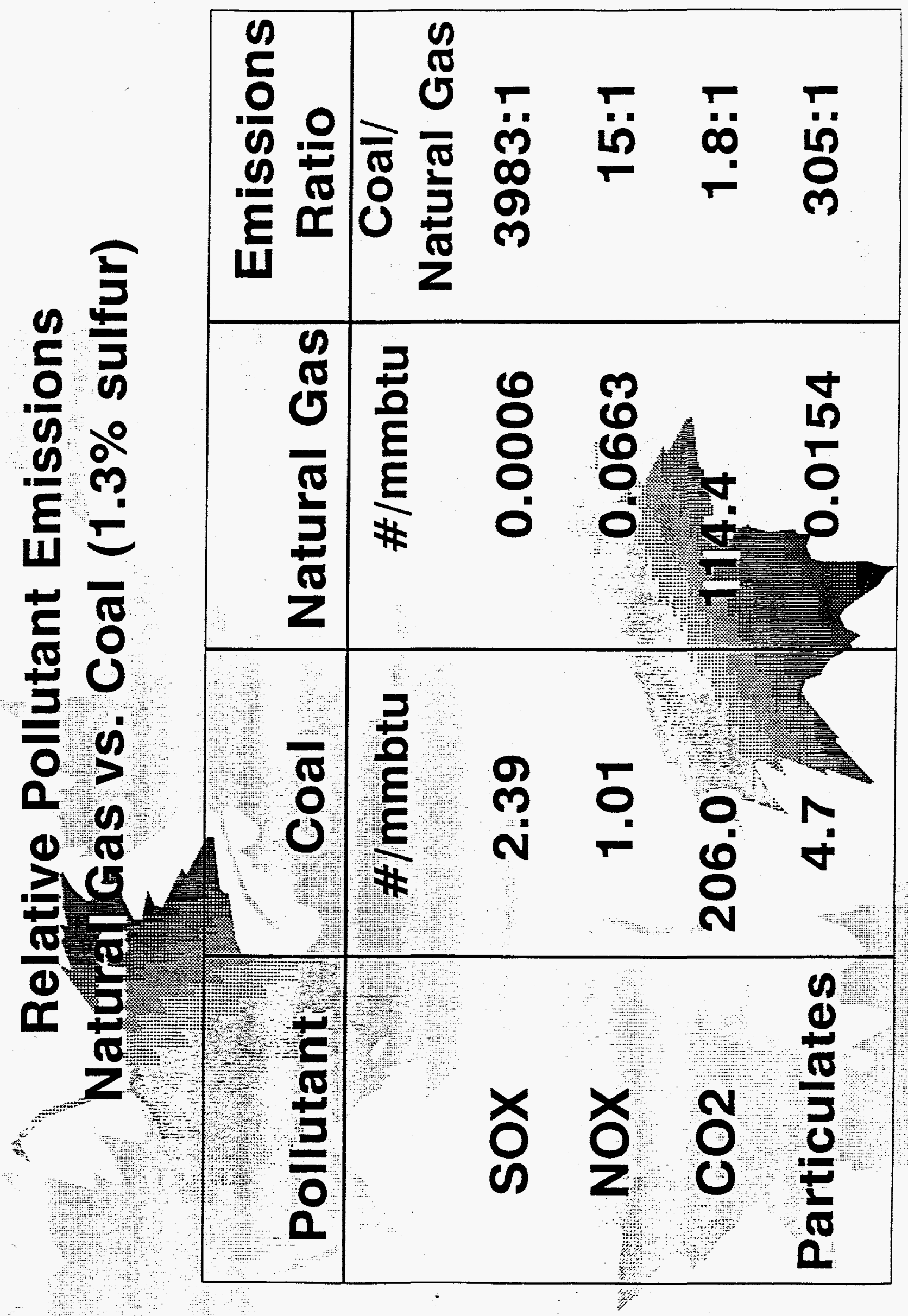

A -183 


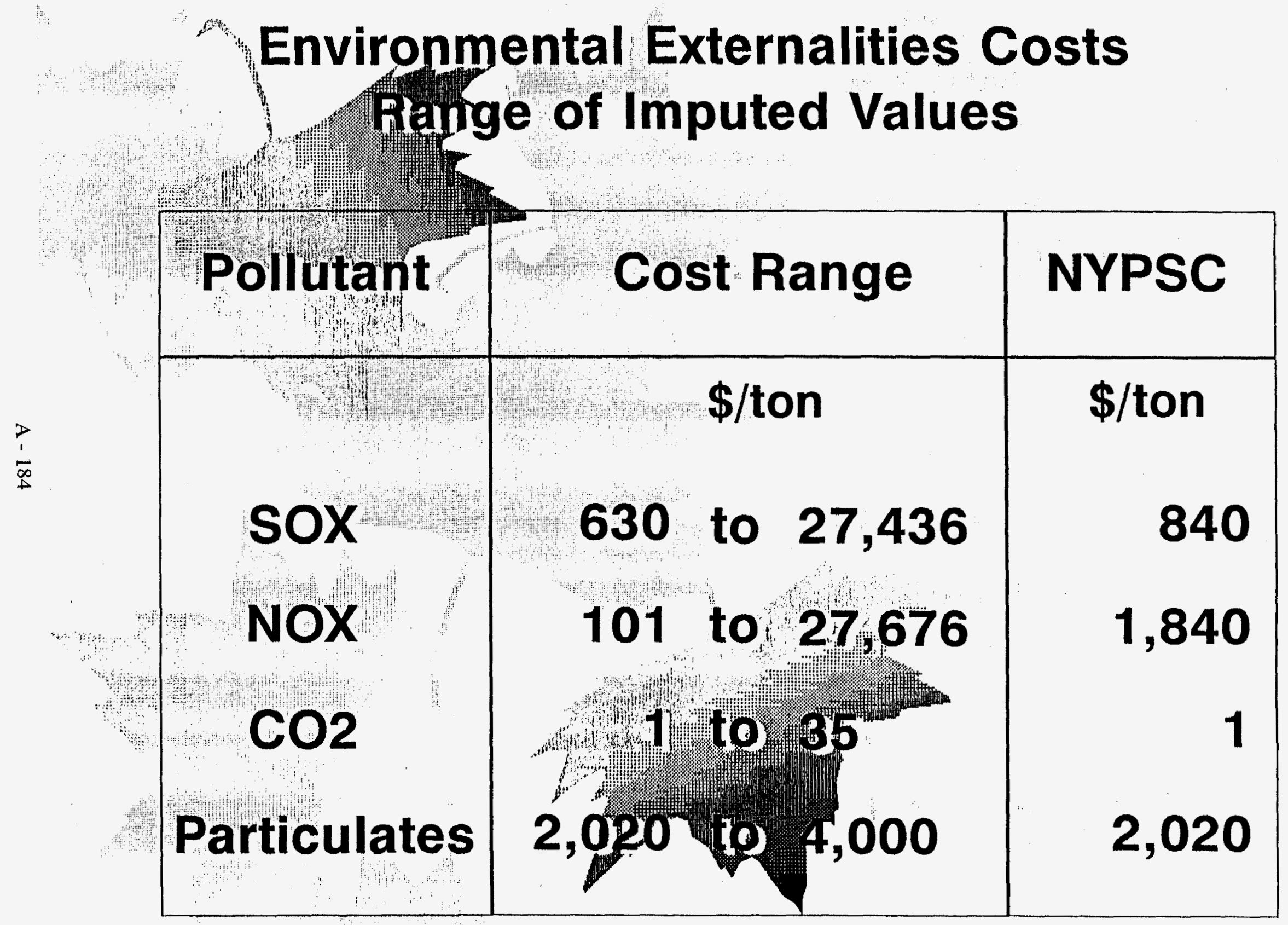




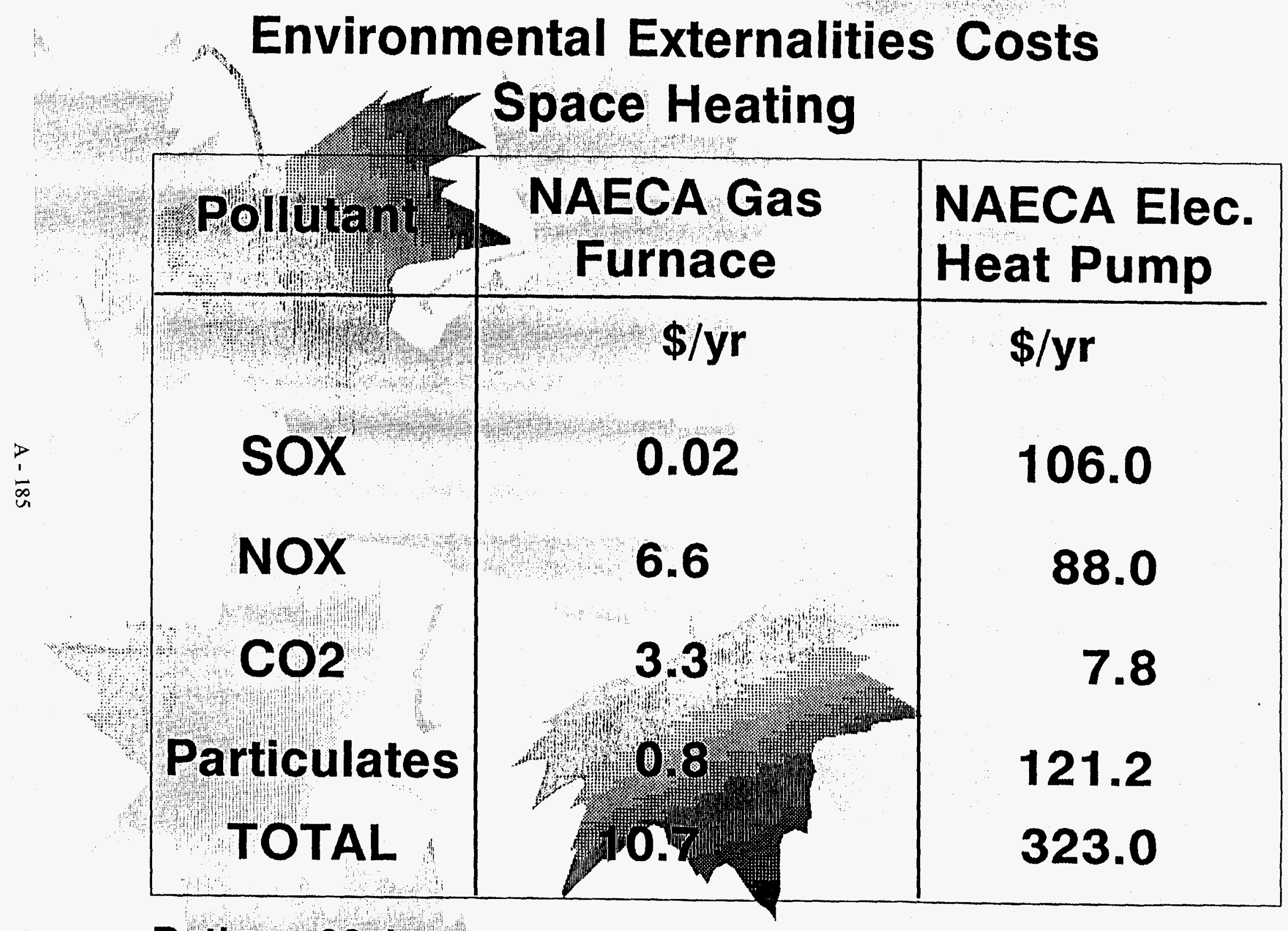

Ratio $=23: 1$ 


\section{Integrated Resource Planning Space Heating Comparison}

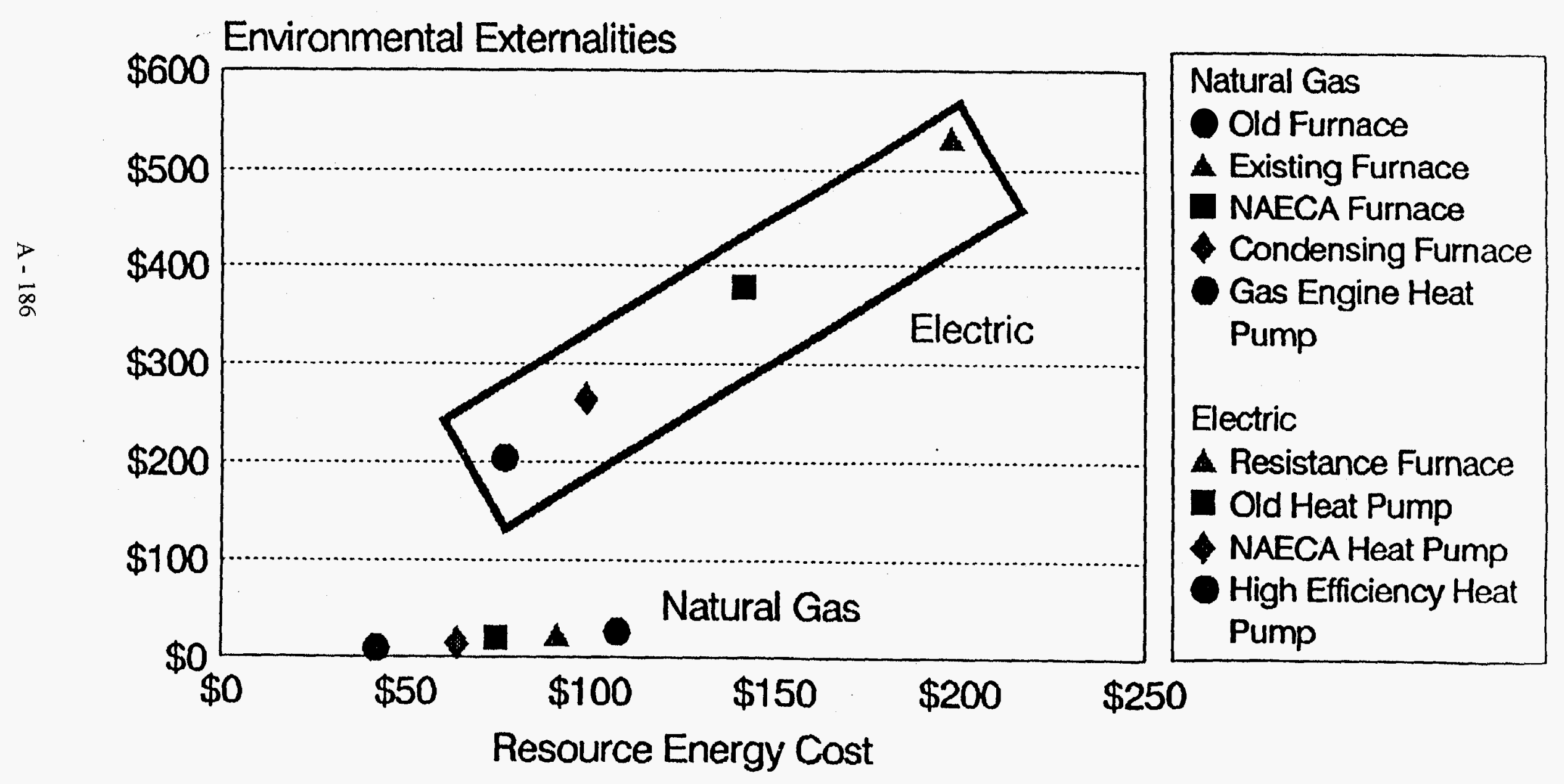



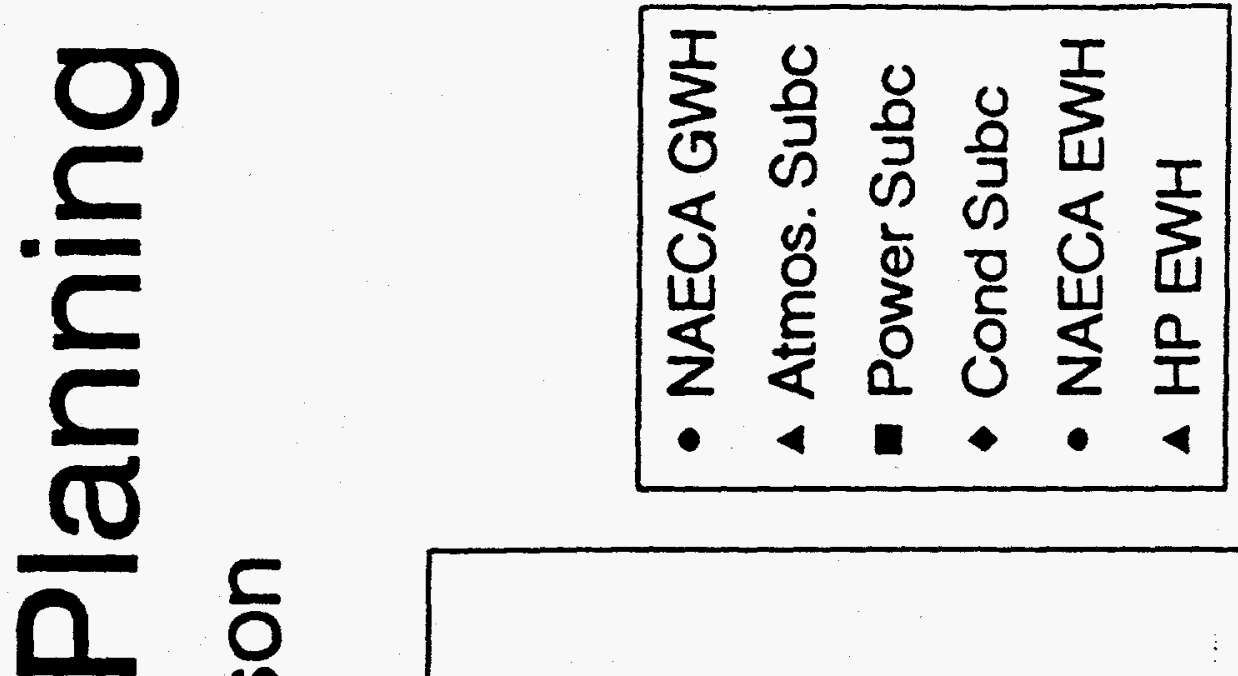

ธ

(4)

ㅇํํ है

O

क
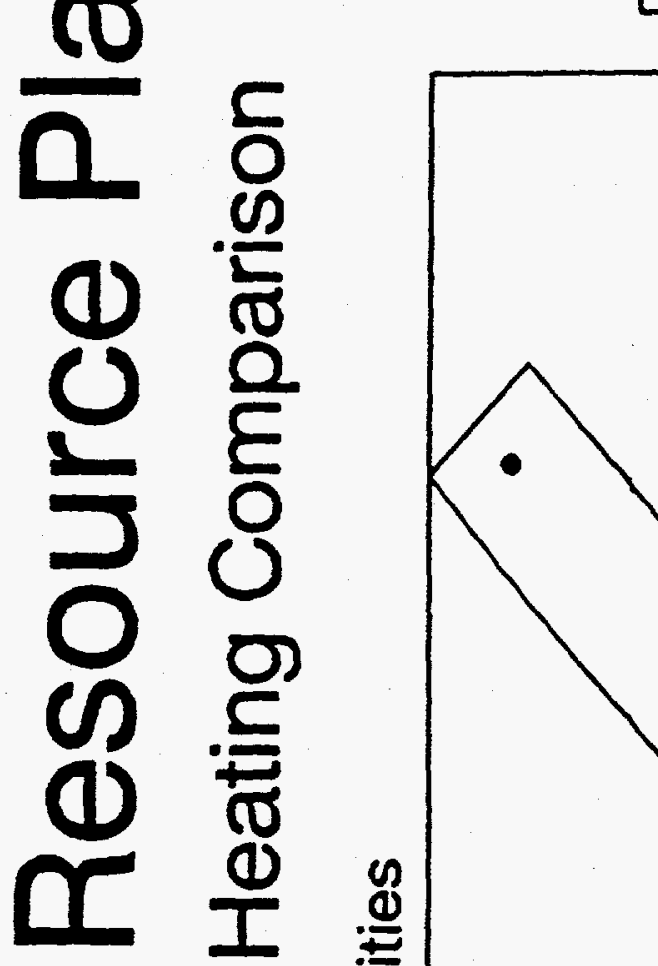

$Q$

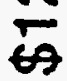

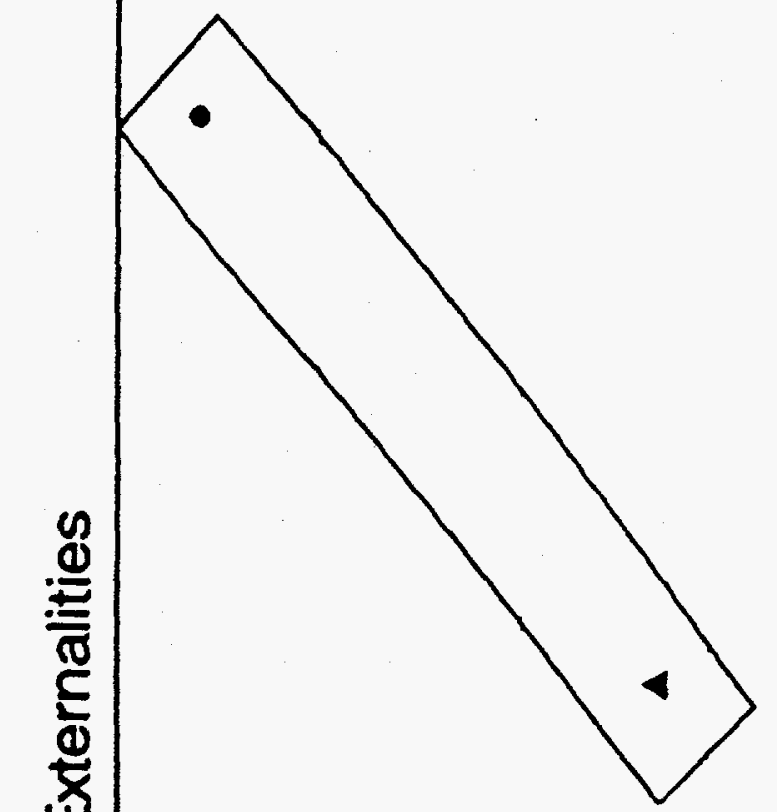

(0)

$\pi$

(ర)

ठั

(1)

도

-

$\frac{1}{0}$
$\frac{E}{5}$
$\frac{2}{2}$
븐

焉

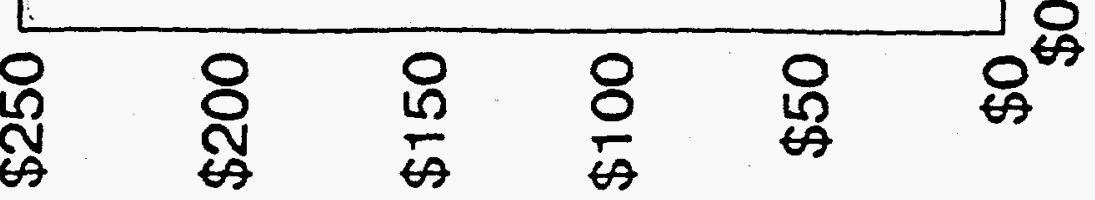

8

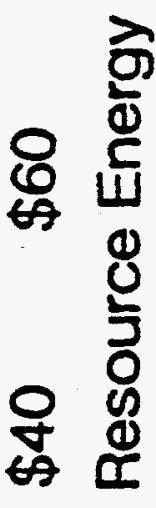

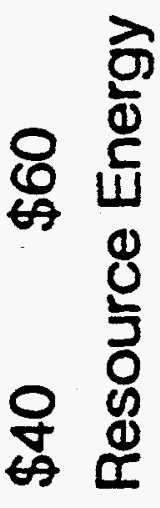

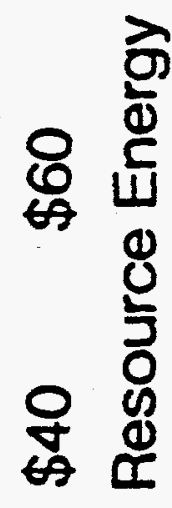

$\frac{0}{6}$

$\hat{Q}$

A -187 


\section{Integrated Resource Planning Commercial Cooling Comparison}

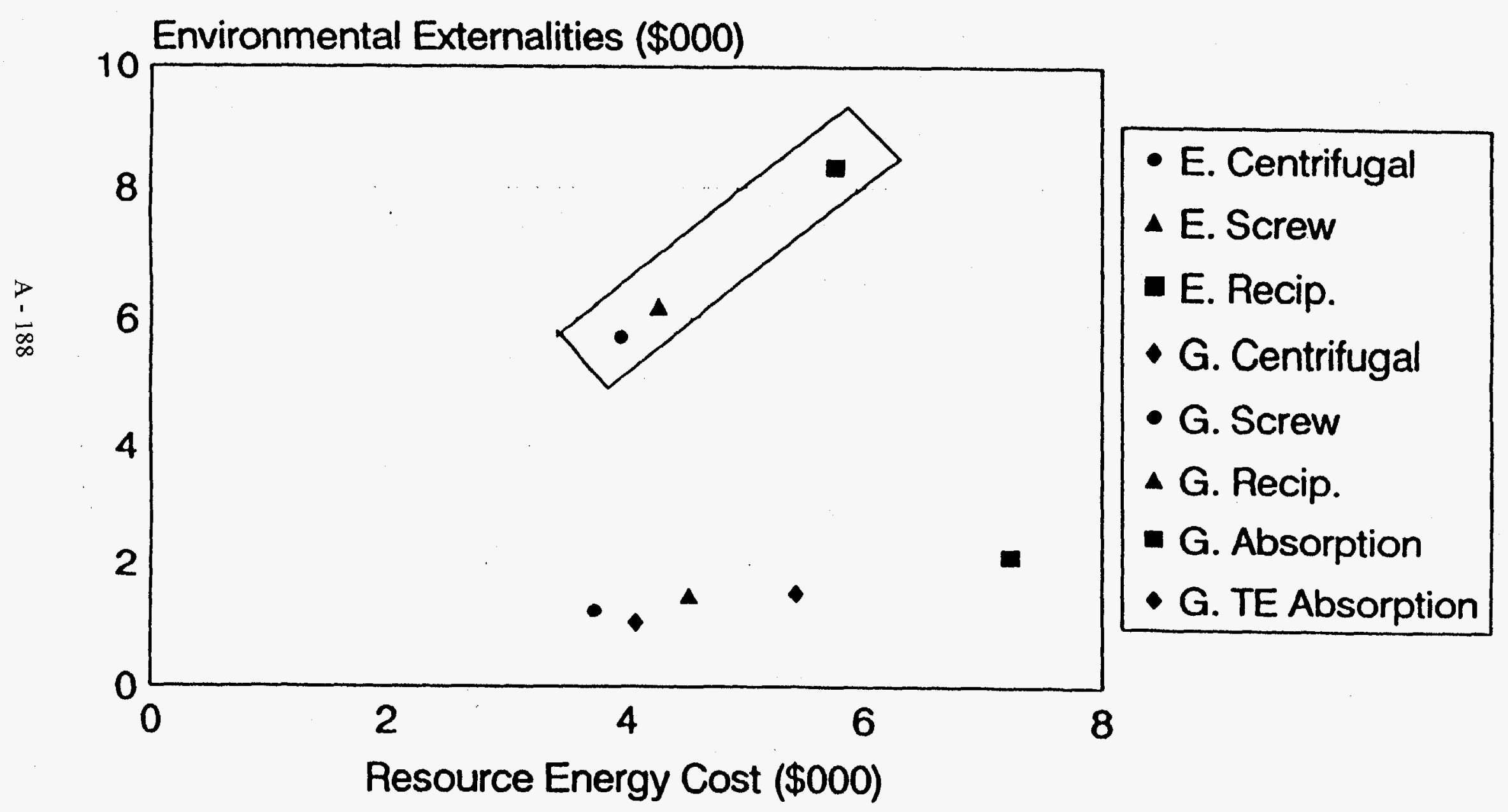

200 RT, 2000 hr. / yr., No heat recovery 


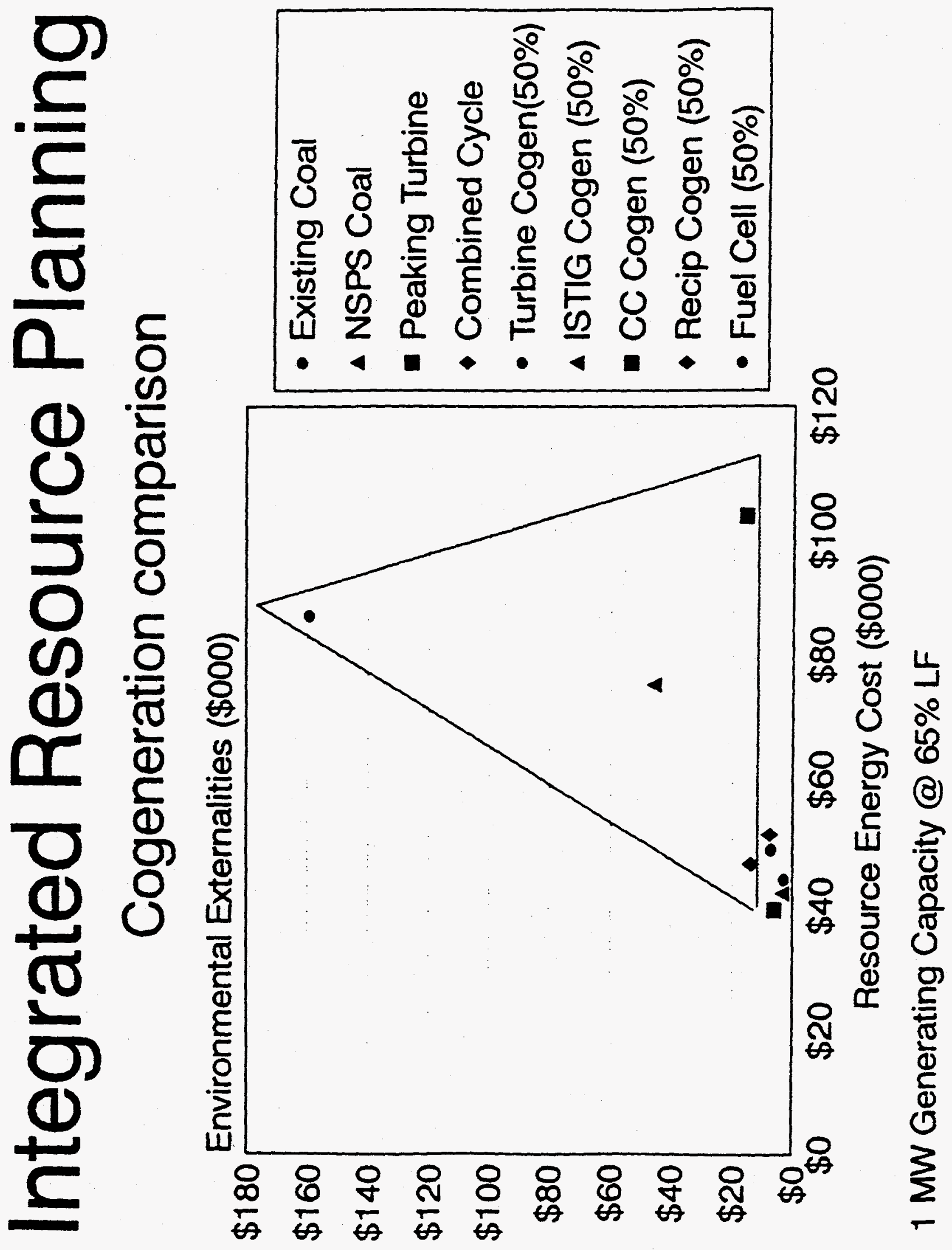


Total Fuel Cycle Analysis Optimal Fuel Selection

- Reduce pollutant emissions

- Reduce emission compliance costs

- Maintain CAAA emissions caps

- Anticipate air toxics regulations

- Defer incremental electric generation

- Conserve resource energy

- Improve national energy sesurity and economic competitiveness 


\section{Integrated Resource Planning Preferred Comprehensive Perspective}

- INTEGRATED:

- all retail energy forms

- simultaneous consideration

- end use optimization

- RESOURCE:

- full trajectory efficiency

- minemouth/wellhead through end use

- environmental sensitivity

- PLANNING:

- concentration on appropriate future development

- full consideration of all available options 
"Generating electricity with natura gas in combined cycle plants currently a major theme in polic circles... If the power comes from gas combined cycle plant all of th space conditioning options hav negligible SO2 emissions...

Following this line of thinking little further, if we accelerat electrification, power generation becomes the largest marginal market for gas, and gas can become a clear transition fuel for power generation.

There are several problems with this policy. What power generation energy sources are we transitioning to as we dry up our gas IDCs and implement total electrification? Nuclear? Wind mills? Photovoltaics? Solar thermal? Geothermal? Second, after we have eliminated the only viable alternative to electricity at end-uses how do we intend to keep electricity bills in check? IRP? Competitive generation markets and open access transmission? Retail Wheeling?... The major problem with the policy is that we will not be able to afford it... 
Instead, we could transform the furnace/air-conditioning to gas heat pumps...To place 6 million gas heat pumps by 2010 would require shipping about 350,000 units per year between now and then. At these volumes gas heat pumps would surely be available at no cost premium over furnace/airconditioners within five years...

In the opinion of the author, policymakers should be encouraging end-use market transformations just as vigorously as they are encouraging gas combined cycle power generation."

"Space Conditioning Options In The North" by Patrick J. Hughes, DSM Quarterly, Summer 1993. 
A -194 
STATE REGULATORY AND PLANNING PERSPECTIVES ON TOTAL FUEL CYCLE ANALYSIS 
A -196 
Total Fuel Cycle Analysis (TFCA) Workshop

\section{Comments by State Representative Robert Earley \\ Chairman, Texas House Committee on Energy Resources}

* Total Fuel Cycle Analysis offers a great deal of potential to:

-Make energy use more efficient

-Help energy policy makers work together

-Lower the total costs of energy use.

- I see Total Fuel Cycle Analysis as an expansion of Integrated Resource Planning that can be a helpful means of providing incentives for the most efficient energy applications.

* Likely benefits of Total Fuel Cycle Analysis in Texas:

-Reduction of unnecessary pollution

-Cheaper, more efficient allocation of resources

-A stronger economy

-A potential model of government and industry cooperation

-TFCA can help government agencies overcome the myopia that sometimes sets in. This global approach can help policy makers avoid unintended consequences

-Greater market incentives for efficiency, rather than just market share.

* Barriers to a practical application of Total Fuel Cycle Analysis:

-Institutional barriers: division of regulatory agencies, especially: The PUC regulates electric utilities while the Railroad Commission regulates gas utilities and producers. Both are sometimes paternalistic and there is often lack of communication.

-Complexity: Coming from a South Texas District where the largest town is called Beeville, it took me a while to understand traffic lights. Just two years ago, I started getting comfortable with acronyms like IRP and DSM. (Of course I was espousing the benefits of IRP to one of my Texas House colleagues, and he turned to me and said that last time he "urped", it was after a really big lunch, and he didn't know if the legislature should encourage such a policy.) Now we're expanding to include TFCA-with implications for a whole world of energy use. We need to figure out how to simply define TFCA and sell it as a simple, intuitive concept.

-Industry Partisanship: The various energy industries need to work on common ground and assumptions that can benefit both industries. Government must by careful to by fuelneutral and judge the efficiency of sources by consistent standards. But the complexity allows manipulation of arguments that could paralyze a legislator or possibly a regulator.

-The competing definition of "cost": The lowest fuel cycle cost in some eyes would not include environmental costs, while in the eyes of some groups, these are the most significant costs. Though it is very subjective, policy makers need more objective information or criteria for quantifying externalities--and to what extent existing "command 
and control" requirements affect the quantification.

-Existing Government Programs: State policy makers in the Texas Legislature are faced with a hodgepodge of incentives and disincentives that often conflict. Uncertainty about the rapidly changing electric and gas utility markets makes Total Fuel Cycle Assessment even more difficult and subjective. We at the state level must catalog and understand the affects of existing regulatory programs, and which must be superseded.

-Example: In transportation fuels, we in Texas have been spending millions trying to promote propane. But nobody bothered to look and realize that the state government was already providing dis-incentives for propane use: we were taxing propane fuel at a rate almost 50\% higher than a comparable amount of gasoline. This is the sort of myopia that must be overcome.

* If we can work together to overcome these problems with more information and consensus, then the road to a more sustainable energy future will be paved a bit smoother. Policy makers can make reasonable decisions, these policies can be sold to the public, and we can turn a brilliant concept into a brilliant example of cooperation. 


\title{
MULTI STAGE EMISSION ACCOUNTING: NEW YORK EXPERIENCE
}

\author{
by \\ Dr. Ajay K. Sanghi \\ Chief, Impact Analysis Unit \\ Division of Policy Analysis and Planning \\ New York State Energy Office \\ Phone (518) 474-7690 \\ Fax (518) 473.8687 \\ Presented At \\ TOTAL FUEL CYCLE ASSESSMENT WORKSHOP \\ sponsored by \\ National Renewable Energy Laboratory \\ and the \\ U.S. Department of Energy \\ In Austin, Texas \\ October 6-7, 1994
}

The views and opinions expressed in this paper are solely those of the author and do not necessarily state or reflect the views, opinions or policies of the New York State Energy Office. 


\section{NEW YORK STATE ENERGY PLAN Ii!!}

- New York State has a comprehensive energy planning process.

- - It represents the first time a State's energy, environmental and regulatory agencies have worked together in developing energy policies to achieve State environmental, energy and economic policy objectives.

- The New York State Energy Plan (NYSEP) is developed jointly by the:

- New York State Energy Office

- New York State Department of Environmental Conservation and

- New York State Department of Public Service.

- The Draft 1994 State Energy Plan was completed in Feb. 1994, the final State Energy Plan is to be released soon. 


\section{MULTI-STAGE EMISSION ACCOUNTING MODEL}

- The multi-stage emission accounting model provides a comprehensive assessment of energy and emission impacts of alternative technologies and scenarios.

- Conventional environmental analysis generally focuses on the emissions associated only with the final combustion stage (i.e. at the end-use level).

- The multi-stage emission accounting model not only estimates emissions at the end-use level, but also accounts for the fuel use and emissions in the extraction, processing and transportation of the fuels consumed at the end-use level (Figure 1 ).

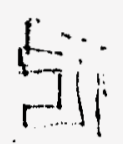




\section{Figure 1}

\section{Multi-Stage Fuel Use and Emissions}

\section{\begin{tabular}{l|l|l|l} 
Extraction & Processing & Transport & Primary Use
\end{tabular}}

Rest of U.S.

Coal Mining $\rightarrow 0$
Rest of World $\quad$ Coal Cleaning




\section{IMPORTANCE OF REGIONAL PERSPECTIVE}

- Regional details concerning fuel use, emissions and economic impacts are important for state energy planning.

- Examples:

- The 1994 Draft NY State Energy Plan shows a variation in $\mathrm{SO}_{2}$ emissions among various electricity scenarios of as much as $\mathbf{3 0 0}$ percent.

Will New York State policy be indifferent among the electricity scenarios just because a national $\mathrm{SO}_{2}$ cap hás been imposed?

- - New York State employment impacts of a photovoltaic facility are about 70 percent greater than employment impacts of a new natural gas combined cycle (NGCC) facility. This difference is negligible for the U.S.

Will New York State policy not favor photovoltaic technology over NGCC technology from an economic development perspective? 


\section{REGIONAL PERSPECTIVE OF MULTI-STAGE EMISSION ACCOUNTING}

- The multi-stage emission accounting model estimates total fuel use and emissions at three different regional levels: New York State, rest of U.S. and rest of the world.

- - Factors which define the fuel use and emissions resulting from extraction, processing and transportation of fuels are estimated at the three regional levels: New York State, rest of U.S., and rest of the world.

- For each of the three regional levels, the factors specify the amounts of additional fuel used and pounds of pollutants emitted in extracting, processing and transporting each unit of energy (Tbtu) consumed at the end-use level. 
Table 1

Fuel Use Factors by Location

\begin{tabular}{|c|c|c|c|c|c|}
\hline \multirow[b]{2}{*}{ Fuel Type } & \multicolumn{4}{|c|}{$\begin{array}{l}\text { Fuel Use Factors in Energy Production Stages } \\
\text { (Extraction. Processing and Transportation) }\end{array}$} & \multirow{2}{*}{$\begin{array}{l}\text { Percent } \\
\text { Increase } \\
\text { Over End } \\
\text { Use Level }\end{array}$} \\
\hline & NY & Rest of US & Rest of World & Total & \\
\hline & \multicolumn{4}{|c|}{ (Tbtu of fueT used for every Tbtu of End-Use FueT) } & \\
\hline Petroleum & 0.0033 & 0.0665 & 0.0639 & 0.1338 & $13.38 \%$ \\
\hline Coal & 0.0194 & 0.0300 & 0.0000 & 0.0494 & $4.94 \%$ \\
\hline Natural Gas & 0.0127 & 0.1709 & 0.0211 & 0.2047 & $20.47 \%$ \\
\hline
\end{tabular}

- Most of the extraction, processing and transportation of fuels occurs outside of New York State.

- Total fuel use could increase by between 5 and 20 percent over end-use fuel consumption. 
Tahle 2

1992 Percentage Increase in $\mathrm{SO}_{2} \mathrm{NO}_{x}$ and $\mathrm{CO}_{2}$ Emissions When Emissions from Production Stage Energy Use are added to End-Use Emissions

\begin{tabular}{|c|c|c|c|c|}
\hline \multirow[b]{2}{*}{ Emission Type } & \multicolumn{3}{|c|}{ Region } & \multirow[b]{2}{*}{ Total } \\
\hline & NY & Rest of US & Rest of World & \\
\hline$\ldots$ & \multicolumn{4}{|c|}{$\bar{\cdots}$ (percent increase over end-use Tevel) $\ldots$} \\
\hline $\mathrm{S} 02$ & $0.24 x$ & $5.31 \%$ & $12.56 \%$ & $18.11 \%$ \\
\hline NOx & $1.34 \%$ & $8.38 \%$ & $11.45 \%$ & $21.18 \%$ \\
\hline $\mathrm{CO2}$ & $0.76 \%$ & $10.78 \%$ & $5.08 \%$ & $16.62 \%$ \\
\hline
\end{tabular}

- Most of the increase in SO2, NOx and $\mathrm{CO} 2$ emissions from production and transportation of fuels occurs outside of New York State.

- Total emissions could increase by between 17 and 21 percent over end-use emissions, depending on the pollutant. 


\section{IMPACTS OF MULTI-STAGE EMISSION ACCOUNTING}

- Use of Multi-stage accounting results in an increase in total emissions over those estimated under conventional end-use level accounting.

- Use of multi-stage Table 3

emission accounting results in a different allocation of emissions between transportation and other energy use sectors than that based on conventional emission accounting. When only in-state emissions are considered, emissions in transportation decrease, while those of other sectors increase.

Percentage Changes of Emissions by Sectors Due to Multi-Stage Emission Accounting (within New York)

\begin{tabular}{lc}
\hline & 1992 \\
S02 (Tons) & $\ldots . .$. \\
Electricity & $0.28 \%$ \\
Buildings & $0.00 \%$ \\
Industry & $0.34 \%$ \\
Transport & $-4.53 \%$ \\
NOx (Tons) & \\
Electricity & $0.89 \%$ \\
Buildings & $1.59 \%$ \\
Industry & $\mathbf{8 . 6 2 \%}$ \\
Transport & $-3.20 \%$ \\
C02 (1000s Tons) & \\
Electricity & \\
Buildings & $0.70 \%$ \\
Industry & $0.82 \%$ \\
Transport & $2.15 \%$ \\
\end{tabular}


- Use of Multi-stage emission accounting can show how, over time, the proportion of total fuel use and emissions associated with energy production stages changes relative to end-use energy and emissions.

- - For example, in New York State between 1992 and 2012 the percentage of $\mathrm{SO}_{2}$ emissions associated with energy production stages is expected to increase (from 18 to 25 percent of end-use emissions). For $\mathrm{NO}_{x}$ the increase is greater (from about 21 to about 45 percent of end-use emissions). For $\mathrm{CO}_{2}$ there is only a negligible increase (from about 17 to about 18 percent of end use emissions).

Table 4

Percentage Increase over Emissions from End-Use Energy when Energy Use in Production Stages is Included: 1992 and 2012 /

\begin{tabular}{lccc}
\hline & $\begin{array}{c}\text { S02 } \\
\text { Emission }\end{array}$ & $\begin{array}{c}\text { N0x } \\
\text { Emission }\end{array}$ & $\begin{array}{c}\text { C02 } \\
\text { Emission }\end{array}$ \\
\hline $\begin{array}{l}\text { Percentage Increase over } \\
\text { End-Use Emissions (1992) }\end{array}$ & $18.1 \%$ & $21.2 \%$ & $16.6 \%$ \\
$\begin{array}{l}\text { Percentage Increase over } \\
\text { End-Use Emissions (2012) }\end{array}$ & $25.4 \%$ & $45.2 \%$ & $17.7 \%$
\end{tabular}




\section{CONCLUSIONS}

- The multi-stage emission accounting analysis in the 1994 New York State Energy Plan is a first attempt to address total fuel cycle issues. Data concerning fuel use and emissions in energy production stages (extraction, processing and transportation) are relatively limited. Therefore the results should be viewed as preliminary.

- About 16 to 17 percent additional $\mathrm{CO}_{2}$ emissions occur outside of $\mathrm{New}$ York as a result of the fuel uses within New York. This could be an important environmental concern. Emissions of $\mathrm{CO}_{2}$ within and outside of New York would have the same global warming effects.

- NYSEO is currently doing a study (funded in part by EPA) of options for reduction of greenhouse gas (GHG) emissions in New York. This study uses a multi-stage emission accounting model in order to include GHG emissions outside New York which result from fuel use in New York.

- The proportion of emissions from energy production stages could grow over time. For example $\mathrm{NO}_{x}$ production stage emissions increase from about 21 percent of end-use emissions in 1992 to about 45 percent of end-use emissions in 2012. 
- The higher estimates under multi-stage emission accounting highlight the fact that environmental benefits of energy efficiency improvements and renewables are underestimated if emissions only at the end-use level are counted. Proper accounting of emission reduction benefits are achieved under the multi-stage emission accounting approach which also accounts for the fuel use and emissions in extraction, processing and transportation of the fuels avoided at the end-use level.

- Multi-stage emission accounting should have sufficient regional details for it to be useful in shaping state energy policies. 


\section{TOTAI FUEI CYCIE ANAIYSIS CAIIFORNIA'S PERSPECTIVE TOM TANTON \\ OCTOBER 7, 1994}


O. DEEINITION OF TERMS

0. PAST EFFORTS TOWARDS TOTAL FUEL CYCLE ANAIYSIS

0 . ONGOING ANAIYSES WHICH NEED TO BE EXPANDED TO TOTAI EUEL CYCLE

0. QUALITATIVE-ASPECTS AS SCREENING

O. IMPORTANCE OF TFCA IN CURRENT DEBATE ABOUT RESTRUCTURING THE ELECTRIC INDUSTRY 
0. TOTAL FUEL CYCLE ANAIYSIS

0. IIFE CYCLE ANALYSIS

0. INDIRECT IMPACTS 
0. COMPLEXITY OF ENERGY SYSTEM, AT LEAST IN CAIIFORNIA

O.INDIRECT (I/O) MAY BE AS IMPORTANT IF NOT MORE SO THAN A PRECISE "MOLECULAR LEVEL" TFCA

0. WITH COMING COMPETITION AND SUBSTITUTABILITY ISSUES BECOME EVEN MORE CLOUDED AND SUBJECT TO "DATA OVERLOAD" 


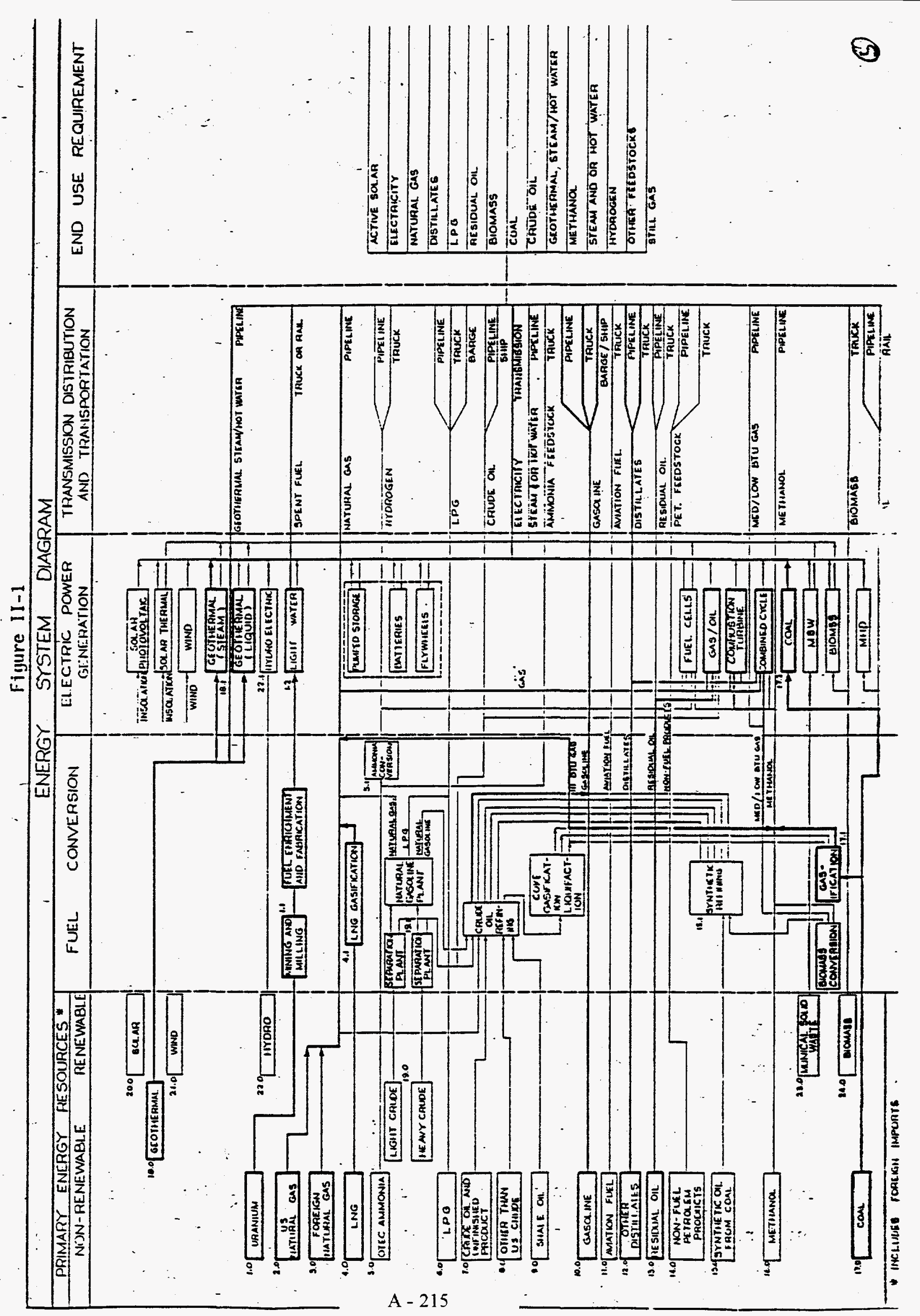


$0 \therefore$ PAST USES INCLUDE:

- GREENHOUSE GAS EMISSION INVENTORY

- EFFICIENCY IMPROVEMENT I\&d TARGETING TO SUPPLY SIDE

- OPPORTUNITY TECHNOLOGY DESIGNATION

- SAFETY STUDIES

0. POINT TO REMEMBER IS THAT THE CONCEPT AND APPICICATION OF TFCA DOES NOT APPLY ONLY TO ENVIRONMETAL IMPACTS 


\section{GREENHOUSE GAS EMISSION INVENTORY}

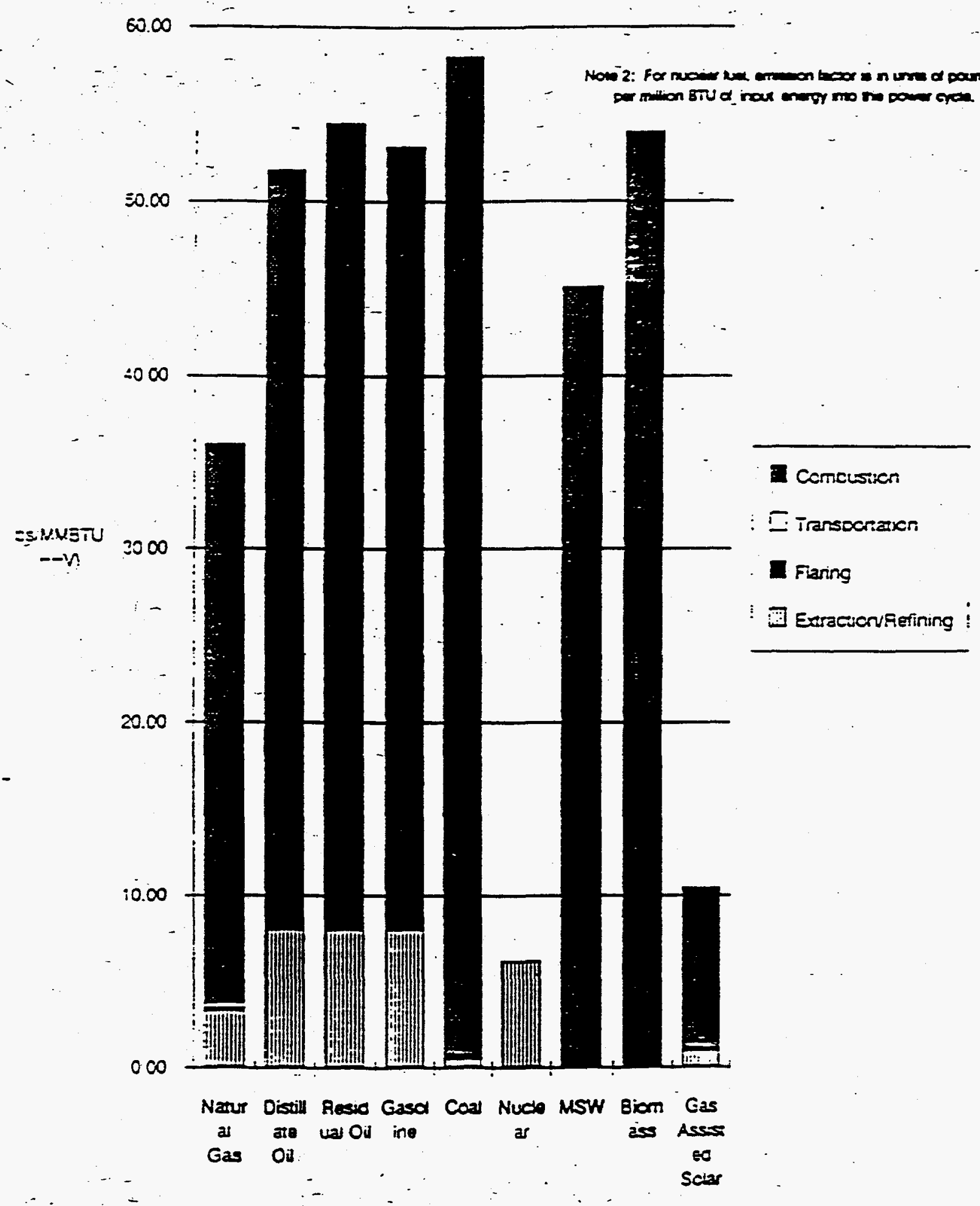


FIGURE B - 5

EFFICIENCY STAIRCASE:

Natural Gas Resources to CNG-Powered Automoblles

100

96

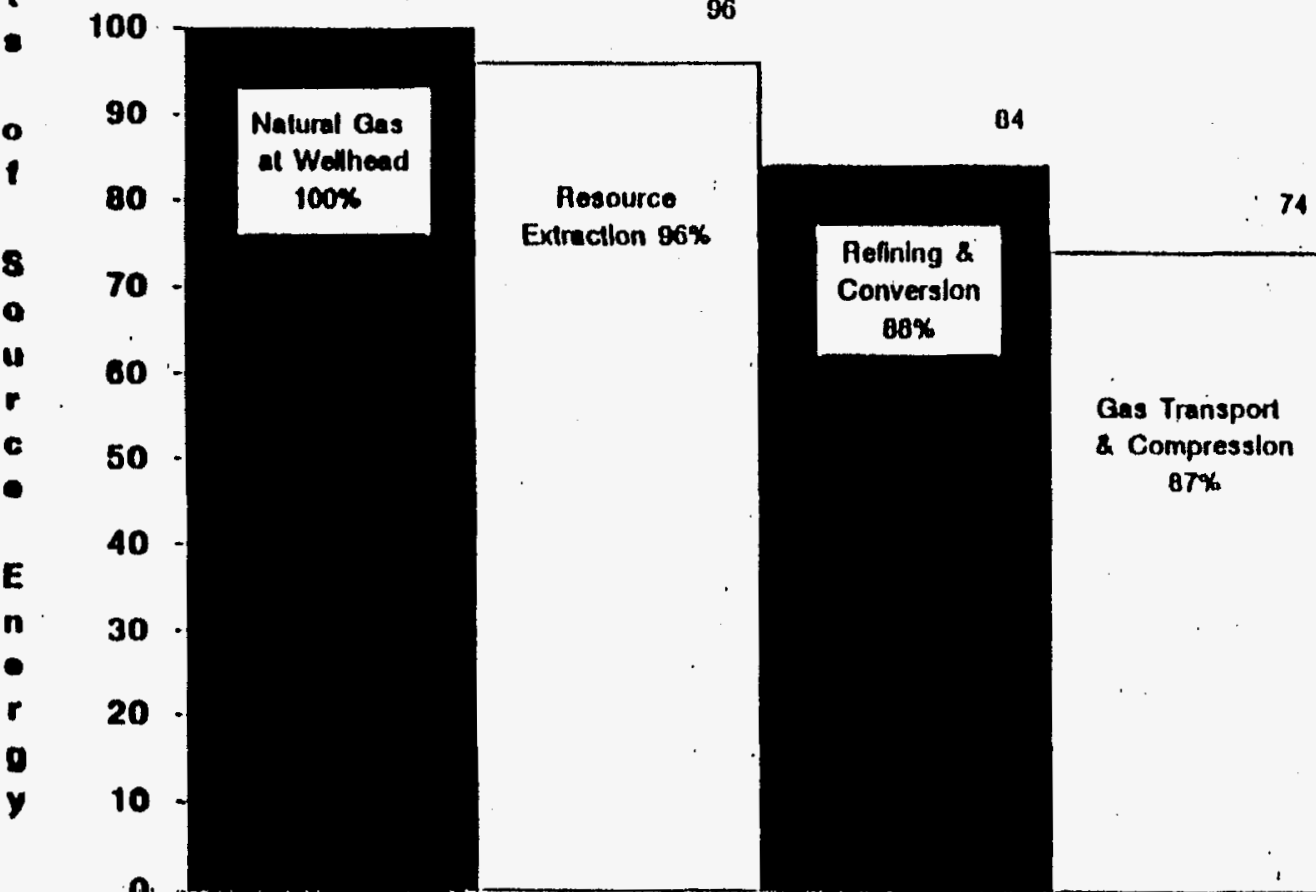

22

Engine

Conversion

10\%

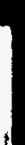

Mochankal

Tranamlasion

$70 \%$

15

7

Aghngi79\% 


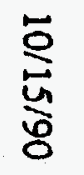

FIGUAE B - 6

EFFICIENCY STAIRCASE:

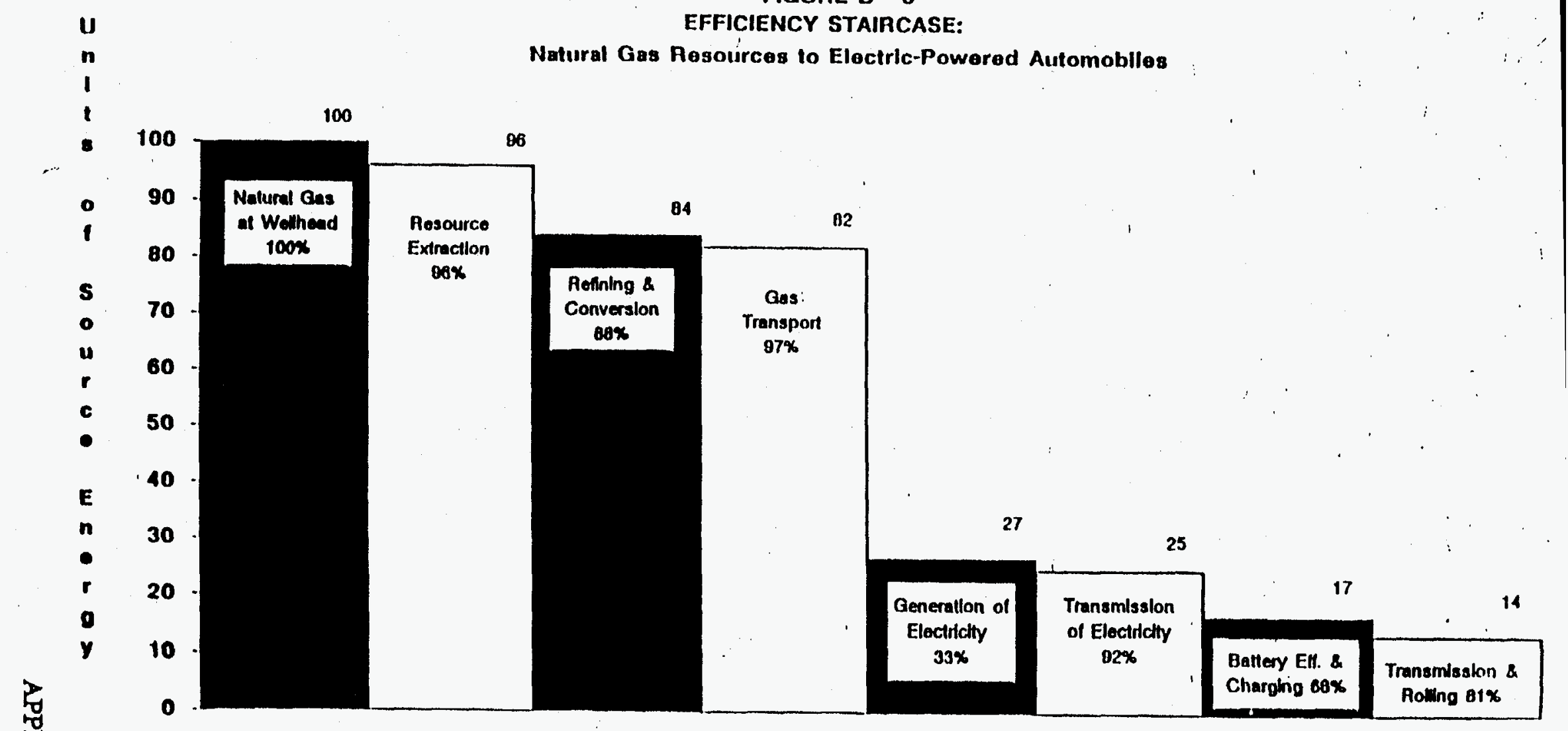


I LLHINULUGYIAPPLICATION:

SCENARIO:

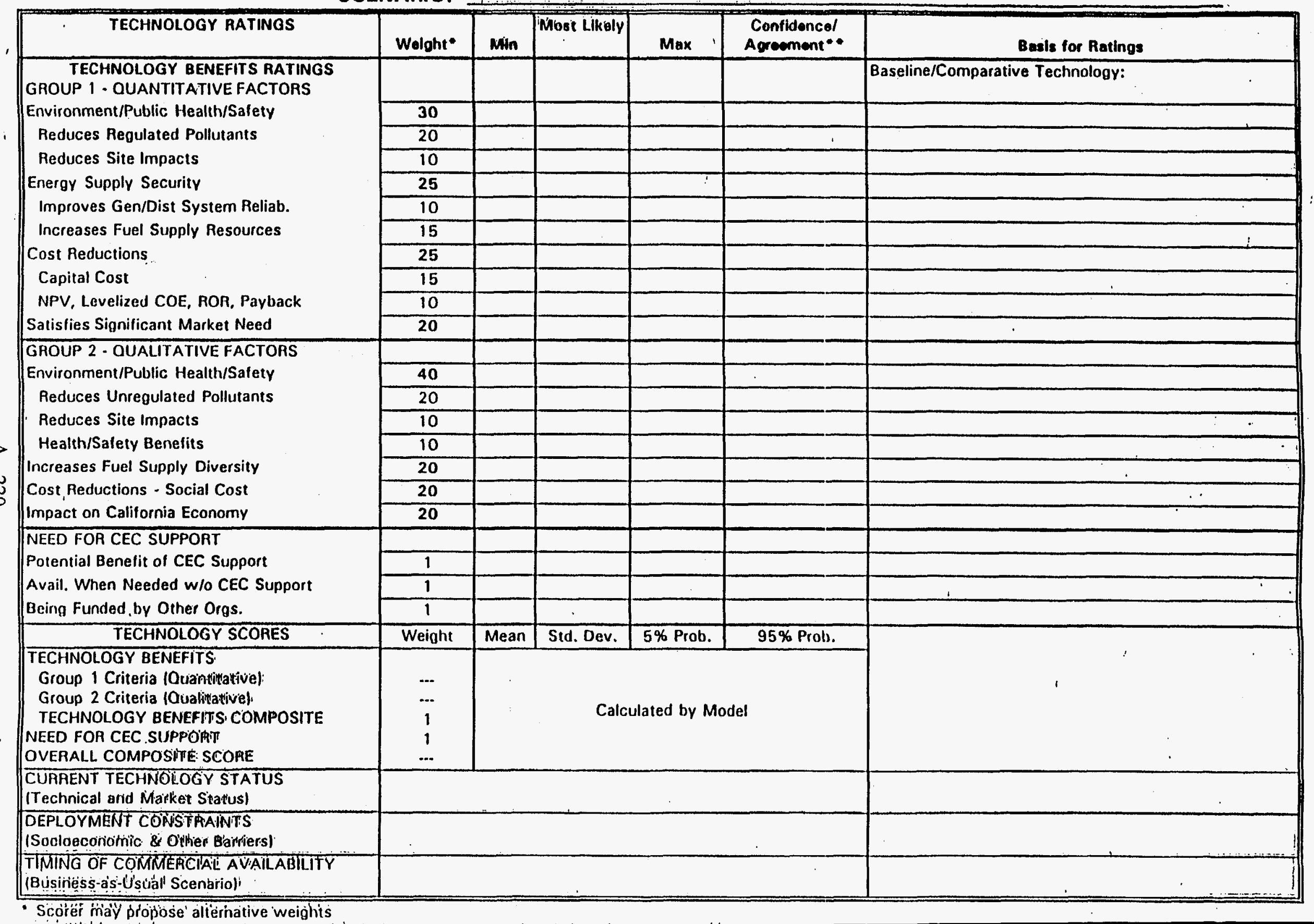

- Scotéé 'mày propose aliériative weights 
0 . ONGOING POLICY ANAIYSES WHICH WOULD BENEFIT EROM TOTAL EUEL CYCIE ANAIYSIS

- DAMAGE FUNCTION ANALYSIS FOR VALUATION OF ENVIRONMENTAL EXTERNALITIES

- CURRENTLY LIMITED TO AIR QUALITY RELATED EXTERNALITIES IN THE ELECTRICITY SECTOR/ AND ONLY AT THE POINT OE ELECTRICITY

PRODUCTION/ (RESULTS APPLIED AS GROSS SCREEN TO TRANSPORTATION ANALYSIS)

- GREENHOUSE GAS EMISSION INVENTORY SHOULD BE EXPANDED TO INCLUDE TECA ON INDIRECT AS WELL AS UPDATING AND EXPANDING EXISTING TECA OF FUEL PORTION. 


\section{FIGURE 3}

\section{ENVIRONMENTAL YALUATION RELATIONSHIPS}

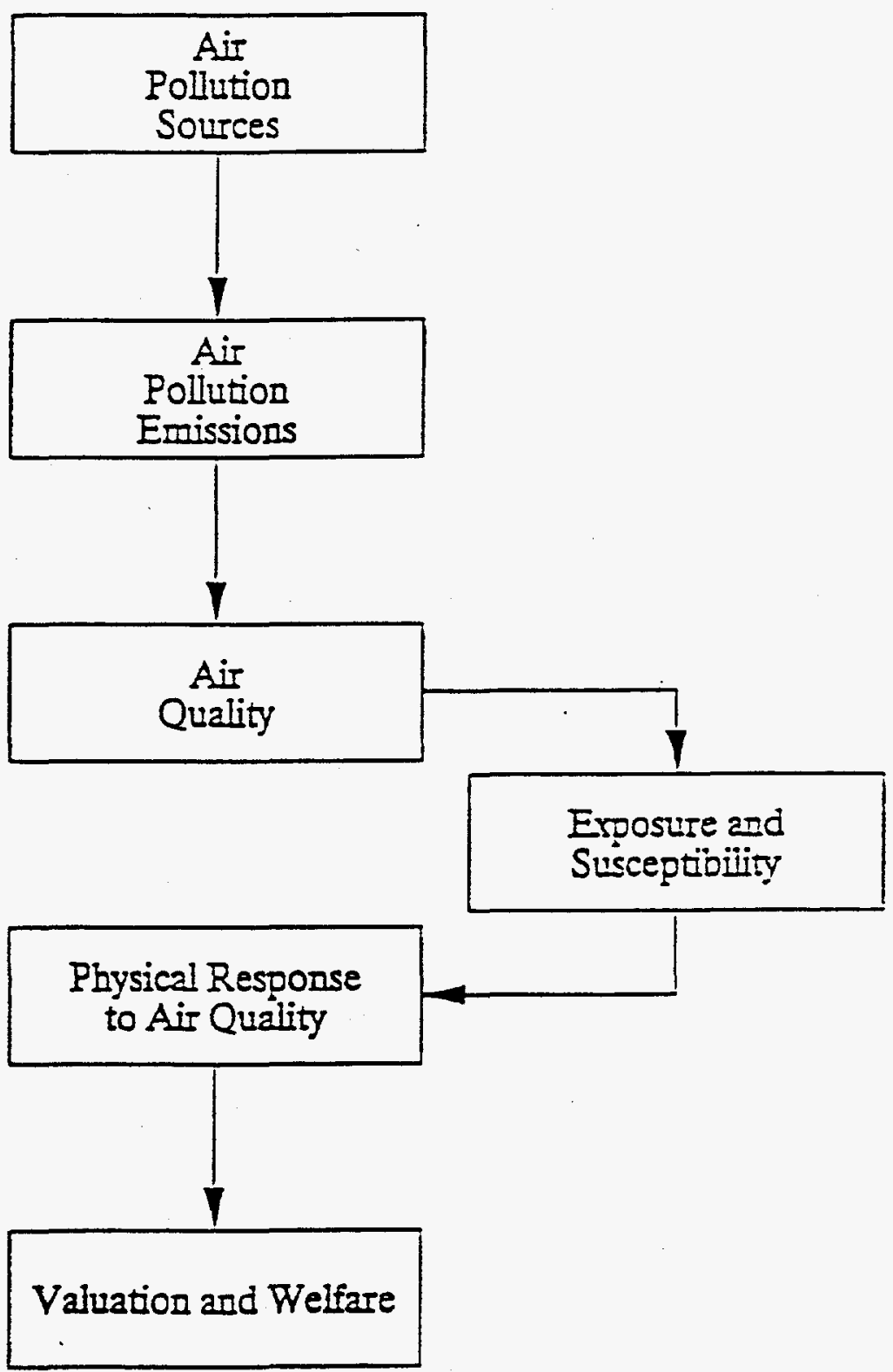


O. ENERGY SYSTEM OF THE FUTURE

0. POTENTIAL IMPACT ON DATA NEEDS FOR TEEA

0 . WILI POLICY MAKERS BE ABLE TO SORT TEREOEGH THE EXPONENTIALIY INCREASING DATA WTPEOTOT COMMITIING EXORBITANT AMOUNTS OE RESOUREES??

O. WHAT LIGHT MIGHT TFCA BRING TO THE DEBATE: ABOUT REMAINING MARKET FAILURES? 

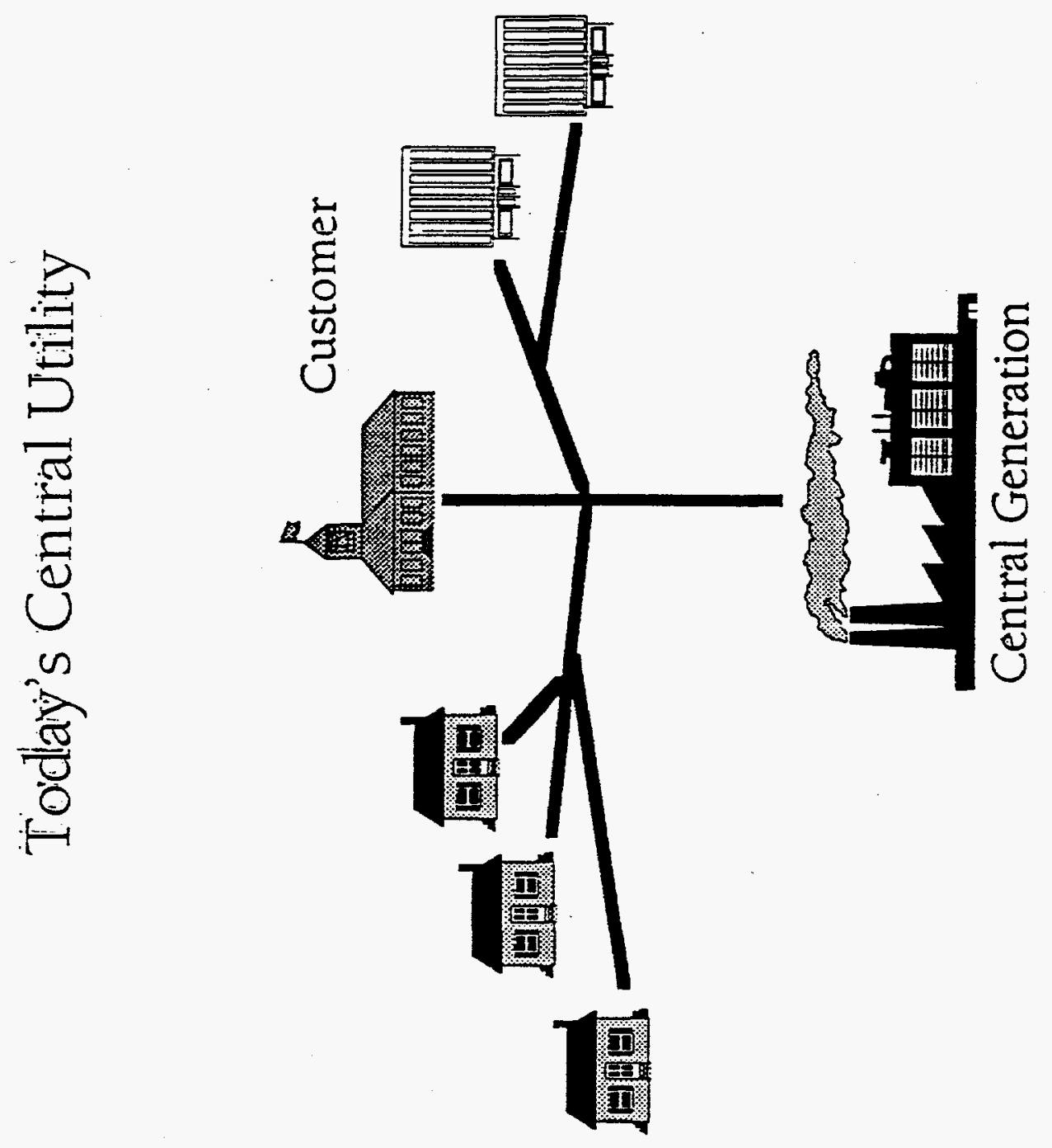

A -224 


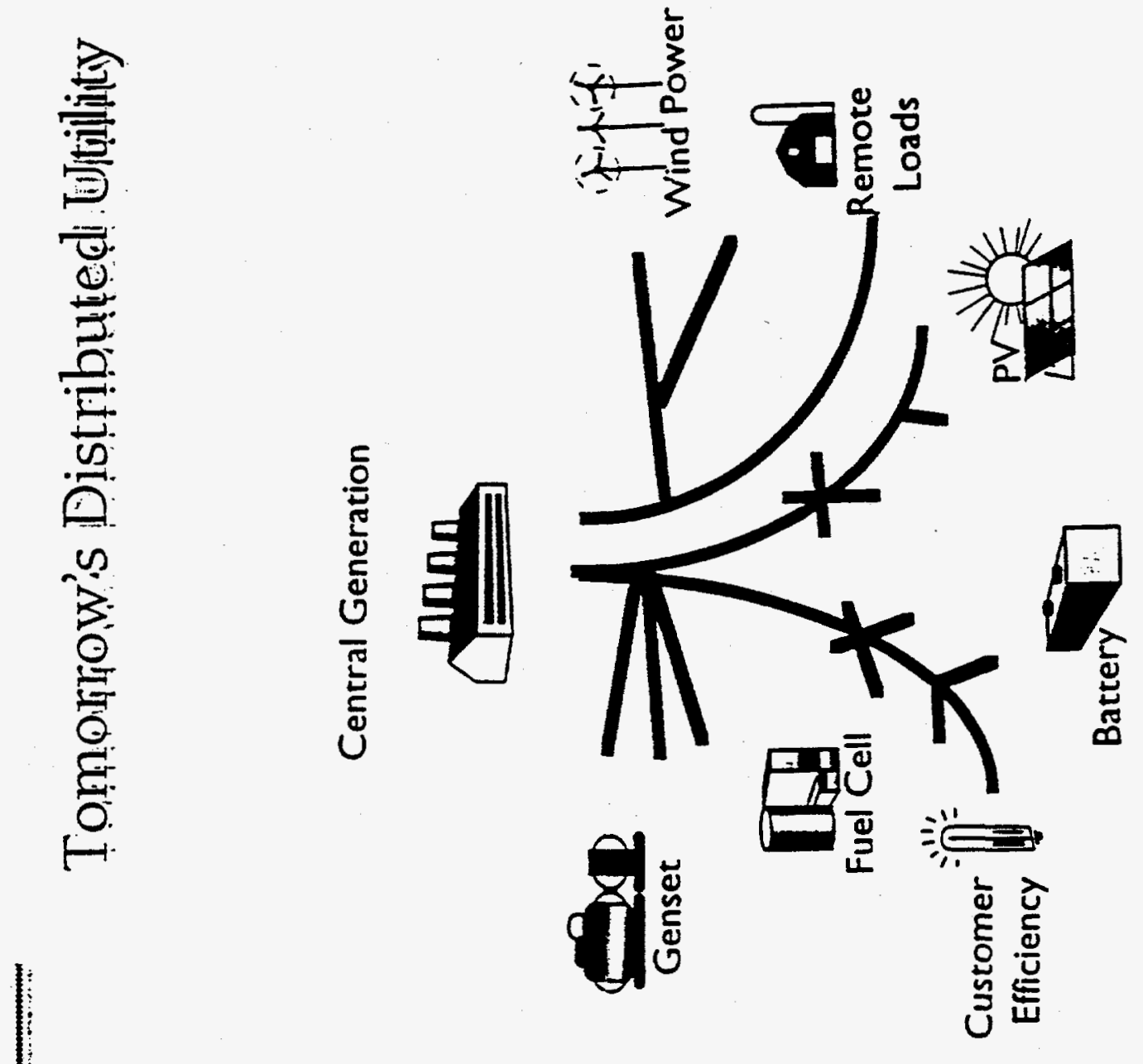


0. FOUR MARKET FAILURES WIIL CONTINUE TO EXIST IN COMPETITIVE FUTURE. TFCA CAN FACELITATE PUBLIC POLICY DEBATE IN SIFTING THROUGH :

1) MONOPOLY: IDENTIEICATION OF REMAINING MONOPOLY "STEPS"

2) EXTERNALITIES: ISOLATION OF TRUE RESIDUAI DAMAGES AND RELATIVE IMPORTANCE OF VARIOUS STEPS AND ALTERNATIVES TO REDUCE IMPACT

3) PUBLIC GOODS: TECHNOLOGY DEVELOPMENT AND SOME DSM

4) COMMON OWNERSHIP: NECESSARY INFRASTRUCTURE AND ??? 
O. BENEEITS OF USING TFCA FOR PUBLIC POIICY ANAIYSIS

- OBVIOUS BENEFIT IS COMPLETENESS AND "TRUTH"

- LESS OBVIOUS BENEFIT IS THAT DECISIONS WILL BE MORE ACCEPTABLE TO PUBLIC AND LESS PRONE TO SECOND GUESSING

- PROVIDES STRUCTURE FOR FOCUSSING ON MAJOR ISSUES IN A RIGOROUS MANNER

0 . COSTS OF USING TFCA

- RESOURCE AND DATA INTENSIVE

- LESS FLEXIBLE TO REAL TIME TECHNOLOGY OR STRUCTURAL CHANGES 
A -228 


\section{SUMMARY SESSIONS:}

KEY POINTS 
A -230 


\section{SUMMARY WORKSHOP SESSIONS: KEY POINTS}

\section{Session No. 1: Establishing Appropriate Boundaries and Parameters for Total Fuel Cycle Assessment}

The discussion generally lacked the coherence and common understanding that is typically associated with a mature and broadly accepted analytical methodology. TFCA is clearly a concept that is in its infancy despite the fact that much has been done over the past two decades to integrate multiple attributes/objectives in regulatory decision making. There was interest on general areas including:

\section{Audience for TFCA and its value}

- If there was any consensus that emerged, TFCA should be a tool for a) state regulators, b) state policy makers, and c) federal policy makers.

- $\quad$ TFCA is described as a tool for doing analysis with the goal of better understanding the breadth and depth of decisions and policies that must consider a wide range of factors that are difficult to integrate.

- $\quad$ Particularly useful in response to an emerging philosophy to "think globally and calculate locally."

- Described as a "comprehensive and rigorous framework for cataloguing decision factors."

- Focus is on three regimes

1) Pre-use - Resource extraction to delivery to the meter

2) Use - Customer value (utility domain)

3) Post-use/Disposal - Not a big issue

- $\quad$ TFCA should concentrate on Pare-use

1) Systematic operations that need to be characterized and described

2) No uniform and/or accepted database

3) Need for a benchmark

- role for DOE

- labor intensive

- objective

\section{Objectives and Attributes}

- $\quad$ Economic efficiency

- Equity and fairness

- $\quad$ Regulatory (environmental) Compliance

- Describe and understand private/internal costs

\section{Simplicity versus Complexity}

- Complexity should be appropriate for the problem or decision at hand

- Generally, the group seemed to form a general consensus around "simpler is better" (even as simple as "counting Btus and emissions"; strong opinion for non-integrated tools) 
There are probably several reasons for this:

1) Analysts tend to seek greater and greater precision that may not contribute significantly to decision processes. This leads to a) diminishing returns, b) greater precision leads to greater complexity, and c) analysts need guidance because they are not directly involved in the decision process.

2) More importantly, however: the clients (PUCs, policy makers) are generally unfamiliar with this tool. Simplicity helps in focusing on the concept. Also, it is more consistent with database accuracy and depth. Simplicity allows the client to understand assumptions. Simple algorithms will lead to a better/quicker understanding and appreciation for how the tool can help (think globally, calculate locally).

\section{Consumer Choice versus Centralized Planning}

- Clearly Recognized

1) Greater trend toward:

- market-based regulation

- greater inter-utility competition

- greater consumer choice

2) The objective is to achieve greater economic efficiency by minimizing costs and optimizing consumer choices and options.

- However, there is still a very robust legacy of 1) policies, 2) regulations and 3) programs put in place under the historical regulatory regime that are probably not appropriate in the future, more competitive environment (especially, IRP and DSM are promotional programs that are based on non-competitive assumptions).

- In this regard, there is strong opinion that TFCA is an appropriate tool (maybe not the only tool) to uncover and highlight unintended consequences of historical market tampering as regulatory paradigms shift towards greater competition and market-based policies.

\section{Possible Role for DOE}

- $\quad$ Policy White Paper and/or regulatory workshops to more fully explore this area.

- How can TFCA be used best in this regard

- $\quad$ Are there other related needs 
- $\quad$ Key points

- Useful for quantifying fuel choice impacts (primary model for this sort of analyses or another needed dimension?)

- Useful for quantifying R\&D funding

- Useful for quantification of emissions \& resource strategies

- Useful for quantification of social costs vs. internal costs of resources (both supply-side \& demand-side)

- Useful for demonstrating "least-cost" alternatives

- Honest

- Accurate

- Relevant

- Easy to use (relatively)

- Comprehensive

- Cost-effective (i.e., avoid diminishing returns)

- Technique (whatever we call it) to figure out distributional effects, cross subsidies, etc.

- Must enhance decision-maker's ability and the overall decision making process

- Data Availability Issues

- Equipment systems

- Are various FERC, EIA, etc. filings adequate?

- Should IPP's EWG's, and/or QF's be required to submit similar filings?

- How do you value miscellaneous by-products (heat recovery, re-cycled coal ash, etc.)

- Should emissions be quantified in terms of mass or monetized?

- Comparability of site vs. remote emissions

- Does the efficiency of renewable resources matter?

- $\quad$ Modeling Approaches \& Issues

- Need equivalent analyses for fragmented "natural gas industry"

- IPP's, NUG's, etc., equivalently fragmented

- T\&D losses for purchased power

- Sales transactions into and out of a given service territory

- $\mathrm{CO} 2$ and methane emissions from coal mining, impurities in wellhead gas, etc.

- Reductions in site emissions vs. increases in remote emissions (total social view)

- Need data on non-point sources and ozone models (preferably hourly)

- Greenhouse gas evaluation

- Temporal, spatial and upstream effects

- Integrate with dispersion models

- Cardinal rule: simplicity is elegant

- Modular approach vs. building a "battleship" 


\section{Session No. 3: Utility Applications of TFCA in Planning}

\section{Major points made by representatives of electric and electric \& gas utilities:}

- Fuel cycle analysis was applied to direct energy use only for the purpose of estimating externalities impacts.

- Externality valuation was imposed by regulators.

- Damage-based approaches were viewed as providing the foundation for sound public policy.

- Reliance on regulated utilities to promote environmental and public policy goals.

- Environmental objectives under discussion:

- command and control

- $\quad$ administered prices

- $\quad$ environmental incentives

- $\quad$ market-based approaches

- Use market-based incentives.

- Recognize uncertainties associated with damages.

- There are externalities that are difficult to quantify.

- Creating fuel cycle analysis and then using adders to quantify emissions is questionable:

- $\quad$ unlikely to improve the environment

- can damage a weak economy

- $\quad$ conflicts with increasing competition

- $\quad$ increases utility rates

- Fuel cycle analysis in the context of competition creates concern:

- piecemeal approach is undesirable

- doesn't reflect the benefits of electrotechnologies

- $\quad$ it is only a mechanistic procedure

- There are concerns over:

- $\quad$ rules applied only to regulated entities

- $\quad$ rigid rules

- Allowance trading should be given consideration

The representative from gas utility represented a different approach:

- Integrated IRP approach

- $\quad$ all retail forms of energy

- simultaneous consideration

- Planning tool

- $\quad$ full consideration of all available options

- allow for an appropriate future development

- $\quad$ Resource tool

- Total fuel cycle analysis is an element in the overall analysis 
- Energy decision making tools should:

- be a platform for decision making

- be a consensus builder for government

- $\quad$ have a regional, national, global framework

- $\quad$ improve communications

- $\quad$ address market failures

- Both New York and California use some form of fuel cycle analysis.

- Data are suspect.

- In New York, it results in options generation -- not policy generation.

- $\quad$ All states were concerned with equity.

- $\quad$ All states were concerned with resource costs.

- States can benefit better information -- not necessarily more information.

- Concerns among state representatives included:

- Impacts

- Economic development

- Competition

- $\quad$ Health effects 


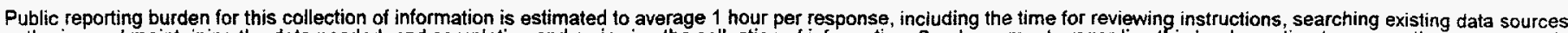
gathering and maintaining the data needed, and completing and reviewing the collection of information. Send comments regarding this burden estimate or any other aspect of this collection of information, inciuding suggestions for reducing this burden, to Washington Headquarters Services, Directorate for inormation Operations and Reports 1215 Jefferso Davis Highway, Suite 1204, Arlington, VA 22202-4302, and to the Office of Management and Budget, Paperwork Reduction Project (0704-0188), Washington, DC 20503.

\begin{tabular}{|l|l|l|}
\hline 1. AGENCY USE ONLY (Leave blank) & $\begin{array}{l}\text { 2. REPORT } \\
\text { DATE. } \\
\text { August 1995 }\end{array}$ & $\begin{array}{l}\text { 3. REPORT TYPE AND DATES COVERED } \\
\text { Subcontractor report }\end{array}$ \\
\hline
\end{tabular}

4. TITLE AND SUBTITLE

Summary and Recommendations: Total Fuel Cycle Assessment Workshop

5. FUNDING NUMBERS

AS115444

6. AUTHOR(S)

BCS, Incorporated

7. PERFORMING ORGANIZATION NAME(S) AND ADDRESS(ES)

BCS, Incorporated

5550 Sternett Place

Suite 216

Columbia, Maryland 21044

9. SPONSORING/MONITORING AGENCY NAME(S) AND ADDRESS(ES)

National Renewable Energy Laboratory

1617 Cole Boulevard

Golden, CO $80401-3393$

10. SPONSORINGIMONITORING AGENCY REPORT NUMBER

TP-463-7671

11. SUPPLEMENTARY NOTES

12a. DISTRIBUTION/AVAILABILITY STATEMENT

National Technical Information Service

U.S. Department of Commerce

5285 Port Royal Road

Springfield, VA 22161
8. PERFORMING ORGANIZATION REPORT NUMBER

13. ABSTRACT (Maximum 200 words)

This report summaries the activities of the Total Fuel Cycle Assessment Workshop held in Austin, Texas, during October 6-7, 1994. It also contains the proceedings from that workshop.

SUBJECT TERMS

fuel cycles; total fuel cycle assessment; utility planning; integrated resource planning

UC

1320

OF REPORT

unclassified

18. SECURITY CLASSIFICATION OF THIS PAGE

unclassified
19. SECURITY CLASSIFICATION OF ABSTRACT unclassified
15. NUMBER OF PAGES 244

16. PRICE CODE

20. LIMITATION OF ABSTRACT UL

Standard Form 298 (Rev. 2-8 Prescribed by ANSI Stc. Z38

NSN 7540-01-280-5500 